\title{
Um método de pontos interiores primal-dual viável para minimização com restrições lineares de grande porte
}

John Lenon Cardoso Gardenghi

DisSERTAÇÃO APRESENTADA

$\mathrm{AO}$

Instituto DE MATEMÁticA E EstatísticA

DA

Universidade de SÃo Paulo

PARA

OBTENÇÃO DO TÍTULO

$\mathrm{DE}$

Mestre em CiênCIAS

Programa: Ciência da Computação

Orientador: Prof. Dr. Ernesto G. Birgin

Durante o desenvolvimento deste trabalho, o autor recebeu auxílio financeiro da Fundação de Amparo à Pesquisa do Estado de São Paulo (FAPESP), processo n ${ }^{\circ}$ 2012/05725-0. 


\title{
Um método de pontos interiores primal-dual viável para minimização com restrições lineares de grande porte
}

\begin{abstract}
Esta versão da dissertação contém as correções e alterações sugeridas pela Comissão Julgadora durante a defesa da versão original do trabalho, realizada em 16/04/2014. Uma cópia da versão original está disponível no Instituto de Matemática e Estatística da Universidade de São Paulo.
\end{abstract}

Banca Examinadora

- Prof. Dr. Ernesto G. Birgin (orientador) - IME-USP

- Prof. Dr. José Mario Martínez Perez - IMECC-Unicamp

- Profa. Dra. Sandra Augusta Santos - IMECC-Unicamp 


\section{Agradecimentos}

Ao Prof. Ernesto, pela orientação, disponibilidade e companheirismo durante o desenvolvimento deste trabalho, bem como pela motivador interesse com que conduziu nossos trabalhos.

Ao Prof. Mario, pelas inestimáveis sugestões dadas ao desenvolvimento deste trabalho, desde sua concepção até o final.

À Profa. Sandra, pela ativa contribuição neste trabalho e durante todo o meu desenvolvimento acadêmico até aqui, desde a minha iniciação científica.

Aos meus amigos, que me proporcionaram momentos de descontração, bem como de reflexão e motivação. Em especial, aos meus amigos do grupo de otimização contínua, pelas sugestões que colaboraram ao desenvolvimento deste trabalho.

Ao meu pai, que sempre me acompanhou, investiu e acreditou em mim. 


\section{Resumo}

Neste trabalho, propomos um método de pontos interiores para minimização com restrições lineares de grande porte. Este método explora a linearidade das restrições, partindo de um ponto viável e preservando a viabilidade dos iterandos. Apresentamos os principais resultados de convergência global, além de uma descrição rica em detalhes de uma implementação prática de todos os passos do método. Para atestar a implementação do método, exibimos uma ampla experimentação numérica, e uma análise comparativa com métodos bem difundidos na comunidade de otimização contínua.

Palavras-chave: programação não linear, restrições lineares, problemas de grande porte, pontos interiores viáveis, busca linear. 


\section{Abstract}

In this work, we propose an interior-point method for large-scale linearly constrained optimization. This method explores the linearity of the constraints, starting from a feasible point and preserving the feasibility of the iterates. We present the main global convergence results, together with a rich description of the implementation details of all the steps of the method. To validate the implementation of the method, we present a wide set of numerical experiments and a comparative analysis with well known softwares of the continuous optimization community.

Keywords: nonlinear programming, linear constraints, large-scale problems, feasible interior-point, line search. 


\section{Sumário}

1 Introdução 1

1.1 Notação e organização deste texto . . . . . . . . . . . . . . . . . . 1

2 Algoritmo de pontos interiores com busca linear 3

2.1 O método . . . . . . . . . . . . . . . . . . . . . . . . 3

2.2 Resolução do subproblema . . . . . . . . . . . . . . . . . . 5

2.3 Resolução do sistema primal-dual . . . . . . . . . . . . . . . . . . . 7

2.4 Modelo algorítmico para a resolução do subproblema . . . . . . . . . . . . . . 9

2.4 .1 Boa definição . . . . . . . . . . . . . . . . . . . . 10

2.4.2 Análise da convergência global . . . . . . . . . . . . . . . . . . 12

2.5 Modelo algorítmico para a resolução do problema . . . . . . . . . . . . . 16

2.5 .1 Boa definição . . . . . . . . . . . . . . . . . . 16

2.5.2 Análise da convergência global . . . . . . . . . . . . . . 17

3 Detalhes de implementação $\quad 19$

3.1 Critérios de parada . . . . . . . . . . . . . . . . . . . . . . . . 19

3.2 Escalamento do problema original . . . . . . . . . . . . . . . . . . . 20

3.3 Computação do ponto inicial . . . . . . . . . . . . . . . . . . . 21

3.3.1 Computação do ponto inicial usando programação linear . . . . . . . . . . . . 22

3.3.2 Computação do ponto inicial resolvendo problemas de viabilidade . . . . . . . . 22

3.4 Estimativa inicial para os multiplicadores de Lagrange . . . . . . . . . . . . . . . . . 23

3.5 Lidando com direções de busca pequenas . . . . . . . . . . . . . . . . . . . . 23

3.6 Correção de inércia . . . . . . . . . . . . . . . . . . . . . . . . . . . 24

4 Experimentos numéricos $\quad 27$

4.1 Parte I - Resultados gerais . . . . . . . . . . . . . . . . . . 27

4.2 Parte II - Análise comparativa . . . . . . . . . . . . . . . . . 31

5 Considerações finais e trabalhos futuros $\quad 37$ 
Apêndices

A Condições de qualificação do problema

Referências Bibliográficas 


\section{Capítulo 1}

\section{Introdução}

Métodos para minimização restrita compõem uma teoria muito ampla e que permanece em constante desenvolvimento. Em particular, problemas de minimização sujeitos a restrições lineares possuem uma série de propriedades teóricas e computacionais que problemas de minimização com restrições gerais - lineares e não lineares - não possuem. Neste contexto, métodos para resolução de problemas não lineares linearmente restritos são amplamente estudados. Muitos dos métodos clássicos para minimização com restrições lineares baseiam-se na consolidada estratégia de restrições ativas, como é o caso do método Simplex para programação linear. Em contrapartida, métodos de pontos interiores surgiram em programação linear e se mostraram competitivos com as estratégias clássicas até então desenvolvidas, inclusive para problemas com função objetivo não linear [15], e começaram a ser empregados na teoria e prática em otimização contínua $[4,10,11,25]$.

Métodos de pontos interiores para programação não linear podem ser divididos em dois grupos [21]. No primeiro grupo, estão os métodos que possuem sólido embasamento nas ideias propostas para programação linear. Estes métodos são baseados em busca linear, em que as direções de busca são determinadas resolvendo-se um sistema de equações lineares através de métodos tradicionais, como o de Newton para sistemas não lineares. Nestes casos, a aceitação e definição do passo são estabelecidos por procedimentos unidimensionais. Por outro lado, temos o segundo grupo, nos quais as direções são obtidas pela minimização de um modelo quadrático, em torno de um ponto, restrito a uma região que garante convergência e globalização, os chamados métodos de regiões de confiança.

Neste trabalho, objetivamos propor um método de pontos interiores para minimização com restrições lineares. A principal característica deste método é que o processo iterativo de otimização deve ser iniciado em um ponto viável e, ao longo do processo, esta viabilidade é preservada. Neste contexto, queremos verificar se uma tal abordagem é válida e competitiva com os métodos correntemente usados na comunidade de otimização.

\subsection{Notação e organização deste texto}

Seja $v \in \mathbb{R}^{n}$ um vetor. Sua $i$-ésima componente é denotada pelo subíndice $v_{i}$, enquanto supraíndices denotam iterandos: $v^{k}$ denota o $k$-ésimo iterando de uma sequência qualquer. Se o vetor possuir um subíndice como parte de seu nome (e.g., $v_{\ell}$ ), então denotamos sua $i$-ésima componente por $\left[v_{\ell}\right]_{i}$. Por outro lado, se $a$ é um escalar, então $a_{k}$ denota o $k$-ésimo iterando. Neste contexto, $i$ denota sempre componentes de vetores, enquanto $j$ e $k$ denotam iterações. Ademais, $e$ representa um vetor, de dimensão apropriada ao contexto, cujas componentes são 1. Os operadores $\|\cdot\|_{1},\|\cdot\|_{\infty} \mathrm{e}\|\cdot\|_{2}$ representam a norma 1, infinito e Euclidiana, respectivamente. 


\section{Capítulo 2}

\section{Algoritmo de pontos interiores com busca linear}

Neste capítulo descreveremos um método de pontos interiores aplicado ao problema

$$
\begin{array}{ll}
\min _{x} & f(x) \\
\text { s. a } & A x=b \\
& \ell \leq x \leq u,
\end{array}
$$

com $x \in \mathbb{R}^{n}, A \in \mathbb{R}^{m \times n}, \ell, u \in(\mathbb{R} \cup\{-\infty,+\infty\})^{n}$ e $m<n$. O método que apresentaremos é inspirado no IPOPT [25]. A principal contribuição deste é a preservação da viabilidade das restrições lineares. Deste modo, este algoritmo se torna competitivo por usar uma abordagem específica para resolução do problema (2.1). Denotaremos por $\mathcal{F}$ o conjunto de pontos viáveis para o problema (2.1), isto é,

$$
\mathcal{F}=\left\{x \in \mathbb{R}^{n} \mid A x=b \text { e } \ell \leq x \leq u\right\} .
$$

Começaremos com a seguinte definição.

Definição 1. O interior relativo do conjunto viável do problema (2.1), denotado por

$$
\mathcal{F}_{0}=\left\{x \in \mathbb{R}^{n} \mid A x=b \text { e } \ell<x<u\right\},
$$

é formado pelos pontos que satisfazem as restrições de igualdade e ao mesmo tempo, estritamente, as restrições de caixa do problema (2.1).

Para o presente método assumiremos que

Hipótese 1. O conjunto $\mathcal{F}_{0}$ é não vazio.

Hipótese 2. A função objetivo $f: \mathbb{R}^{n} \rightarrow \mathbb{R}$ é contínua e pelo menos duas vezes diferenciável.

Além disso, sem perda de generalidade, suporemos que $A$ possui posto linha completo. De fato, caso isto não ocorra, a Hipótese 1 nos garante que o sistema $A x=b$ é compatível e, portanto, é possível eliminar suas equações redundantes e redefiní-lo.

\subsection{O método}

Consideremos $\mathcal{I}_{\ell}$ e $\mathcal{I}_{u}$ conjuntos de índices tais que

$$
\mathcal{I}_{\ell}=\left\{i: \ell_{i} \neq-\infty\right\} \text { e } \mathcal{I}_{u}=\left\{i: u_{i} \neq \infty\right\}
$$


As condições KKT do problema (2.1) são

$$
\begin{aligned}
\nabla f(x)+A^{T} \lambda-\nu_{\ell}+\nu_{u} & =0 \\
A x-b & =0 \\
x_{i}-\ell_{i} & \geq 0, \forall i \in \mathcal{I}_{\ell} \\
u_{i}-x_{i} & \geq 0, \forall i \in \mathcal{I}_{u} \\
{\left[\nu_{\ell}\right]_{i} } & \geq 0, \forall i \in \mathcal{I}_{\ell} \\
{\left[\nu_{u}\right]_{i} } & \geq 0, \forall i \in \mathcal{I}_{u} \\
{\left[\nu_{\ell}\right]_{i}\left(x_{i}-\ell_{i}\right) } & =0, \forall i \in \mathcal{I}_{\ell} \\
{\left[\nu_{u}\right]_{i}\left(u_{i}-x_{i}\right) } & =0, \forall i \in \mathcal{I}_{u}
\end{aligned}
$$

onde $\lambda \in \mathbb{R}^{m}$ é o vetor de multiplicadores de Lagrange associados às restrições de igualdade e $\nu_{\ell}, \nu_{u} \in$ $\mathbb{R}^{n}$ são os vetores de multiplicadores de Lagrange associados às restrições de caixa. Consideraremos que $\left[\nu_{\ell}\right]_{i}=0$, para cada $i \notin \mathcal{I}_{\ell}$ e $\left[\nu_{u}\right]_{i}=0$, para cada $i \notin \mathcal{I}_{u}$. Como o conjunto viável do problema (2.1) é descrito por restrições lineares, sem qualquer hipótese extra este conjunto já é qualificado, no sentido que todo minimizador local cumpre as condições de Karush-Kuhn-Tucker (KKT). Além disso, a Hipótese 1 implica que todo ponto viável do problema satisfaz a condição de qualificação de Mangasarian-Fromovitz (MFCQ), o que implica que, para todo ponto $x$ que satisfaz as condições KKT, o conjunto de multiplicadores de Lagrange associado é limitado (veja Apêndice A).

O método consiste em resolver aproximadamente uma sequência de subproblemas da forma

$$
\min _{x} f(x)-\mu_{k} \sum_{i \in \mathcal{I}_{\ell}} \log \left(x_{i}-\ell_{i}\right)-\mu_{k} \sum_{i \in \mathcal{I}_{u}} \log \left(u_{i}-x_{i}\right) \text { s. a } A x=b,
$$

com $\mu_{k}$ o parâmetro barreira, positivo para todo $k$, de tal maneira que

$$
\lim _{k \rightarrow \infty} \mu_{k}=0 \text {. }
$$

Às iterações que definem a sequência de subproblemas chamaremos iterações externas, enquanto às iterações despendidas para resolver o $k$-ésimo subproblema chamaremos iterações internas. Neste contexto, utilizaremos como ponto inicial para o $k$-ésimo subproblema a solução aproximada do subproblema $k-1$, de modo que a estimativa inicial para o primeiro subproblema será o ponto inicial calculado durante o pré-processamento (ver Seção 3.3 adiante). As condições de Lagrange do subproblema (2.4) são:

$$
\begin{aligned}
\nabla f(x)+A^{T} \lambda-\mu_{k} L^{\dagger} e+\mu_{k} U^{\dagger} e & =0 \\
A x-b & =0 .
\end{aligned}
$$

onde $L$ e $U$ são matrizes diagonais de ordem $n$ tais que

$$
L_{i, i}=\left\{\begin{array}{cl}
x_{i}-\ell_{i}, & \text { se } i \in \mathcal{I}_{\ell} \\
0, & \text { em caso contrário }
\end{array} \text { e } U_{i, i}=\left\{\begin{array}{cl}
u_{i}-x_{i}, & \text { se } i \in \mathcal{I}_{u} \\
0, & \text { em caso contrário }
\end{array}\right.\right.
$$

e $L^{\dagger}$ e $U^{\dagger}$ são suas pseudoinversas, de forma que

$$
L_{i, i}^{\dagger}=\left\{\begin{array}{cl}
\frac{1}{x_{i}-\ell_{i}}, & \text { se } i \in \mathcal{I}_{\ell} \\
0, & \text { em caso contrário }
\end{array} \quad \text { e } U_{i, i}^{\dagger}=\left\{\begin{array}{cl}
\frac{1}{u_{i}-x_{i}}, & \text { se } i \in \mathcal{I}_{u} \\
0, & \text { em caso contrário. }
\end{array}\right.\right.
$$

Se considerarmos variáveis auxiliares

$$
\left[z_{\ell}\right]_{i}=\left\{\begin{array}{cll}
\frac{\mu_{k}}{x_{i}-\ell_{i}}, & \text { se } i \in \mathcal{I}_{\ell} \\
0, & \text { em caso contrário }
\end{array} \quad \text { e }\left[z_{u}\right]_{i}=\left\{\begin{array}{cl}
\frac{\mu_{k}}{u_{i}-x_{i}}, & \text { se } i \in \mathcal{I}_{u} \\
0, & \text { em caso contrário }
\end{array}\right.\right.
$$


ou, matricialmente, $L Z^{\ell} e-\mu_{k} e=0$ e $U Z^{u} e-\mu_{k} e=0, \operatorname{com} Z^{\ell}=\operatorname{diag}\left(z_{\ell}\right)$ e $Z^{u}=\operatorname{diag}\left(z_{u}\right)$, podemos reescrever (2.5) como

$$
\begin{aligned}
\nabla f(x)+A^{T} \lambda-z_{\ell}+z_{u} & =0 \\
A x-b & =0 \\
L z_{\ell}-\mu_{k} e & =0 \\
U z_{u}-\mu_{k} e & =0
\end{aligned}
$$

Encontrar um $x^{k}$ que resolva aproximadamente (2.4) equivale a encontrar $\left(x^{k}, \lambda^{k}, z_{\ell}^{k}, z_{u}^{k}\right)$ que satisfaça aproximadamente (2.8). Na prática, as equações (2.7) não são usadas; o método consiste em encontrar soluções aproximadas para o sistema (2.8) fazendo $\mu_{k}$ tender a zero. O fato da função objetivo de $(2.4)$ não estar definida para $x \notin(\ell, u)$ e ir para o infinito quando alguma componente de $x$ se aproxima de $\ell$ ou de $u$ implica que $\ell<x^{k}<u$. Este fato, as equações (2.8c) e (2.8d) e a positividade de $\mu_{k}$ implicam que $\left[z_{\ell}^{k}\right]_{i} \geq 0$, para todo $i \in \mathcal{I}_{\ell}$ e $\left[z_{u}^{k}\right]_{i} \geq 0$ para todo $i \in \mathcal{I}_{u}$. Notemos que a resolução de uma sequência de equações do tipo (2.8) com $\mu_{k}$ tendendo para zero pode ser vista como um método homotópico para resolver as condições KKT (2.3) perturbadas. Neste contexto, $z_{\ell}^{k}$ e $z_{u}^{k}$ assim definidos são aproximações dos multiplicadores de Lagrange $\nu_{\ell}$ e $\nu_{u}$ do problema original (2.1), mais exatas quanto menor for o valor de $\mu_{k}$.

$\mathrm{Na}$ seção a seguir, apresentaremos a estratégia para a resolução dos subproblemas.

\subsection{Resolução do subproblema}

Na $k$-ésima iteração externa, definimos

$$
\varphi_{\mu_{k}}(x) \stackrel{\text { def }}{=} f(x)-\mu_{k} \sum_{i \in \mathcal{I}_{\ell}} \log \left(x_{i}-\ell_{i}\right)-\mu_{k} \sum_{i \in \mathcal{I}_{u}} \log \left(u_{i}-x_{i}\right)
$$

e consideramos o problema

$$
\begin{array}{ll}
\min _{x} & \varphi_{\mu_{k}}(x) \\
\text { s. a } & A x=b .
\end{array}
$$

Pressupomos que temos $\left(x^{k, j}, \lambda^{k, j}, z_{\ell}^{k, j}, z_{u}^{k, j}\right)$ na $j$-ésima iteração interna, com $j \geq 0, \operatorname{com} x^{k, j} \in \mathcal{F}_{0}$, $z_{\ell}^{k, j}$ e $z_{u}^{k, j}$ positivos e descreveremos uma iteração de um método de busca linear para calcular $\left(x^{k, j+1}, \lambda^{k, j+1}, z_{\ell}^{k, j+1}, z_{u}^{k, j+1}\right)$ tal que $x^{k, j+1} \in \mathcal{F}_{0},\left[z_{\ell}^{k, j+1}\right]_{i}>0$ para cada $i \in \mathcal{I}_{\ell}$ e $\left[z_{u}^{k, j+1}\right]_{i}>0$ para cada $i \in \mathcal{I}_{u}$. Para tanto, a cada iteração deverá ser calculada uma direção $\left(d_{x}^{k, j}, d_{\lambda}^{k, j}, d_{z_{\ell}}^{k, j}, d_{z_{u}}^{k, j}\right)$, com $d_{x}^{k, j}$ de descida para $\varphi_{\mu_{k}}(\cdot)$ a partir de $x^{k, j}$, e um passo $\alpha_{k, j}$ tal que a condição de Armijo dada por

$$
\varphi_{\mu_{k}}\left(x^{k, j}+\alpha_{k, j} d_{x}^{k, j}\right) \leq \varphi_{\mu_{k}}\left(x^{k, j}\right)+\gamma \alpha_{k, j} \nabla \varphi_{\mu_{k}}\left(x^{k, j}\right)^{T} d_{x}^{k, j}
$$

seja satisfeita, para algum $\gamma \in(0,1)$.

As condições de otimalidade do problema (2.10) são dadas por (2.8). A direção de Newton para o sistema (2.8) em $\left(x^{k, j}, \lambda^{k, j}, z_{\ell}^{k, j}, z_{u}^{k, j}\right)$ é a solução do sistema linear de dimensão $3 n+m$ dado por

$$
\left(\begin{array}{cccc}
\nabla^{2} f\left(x^{k, j}\right) & A^{T} & -I & I \\
A & 0 & 0 & 0 \\
Z_{k, j}^{\ell} & 0 & L_{k, j} & 0 \\
-Z_{k, j}^{u} & 0 & 0 & U_{k, j}
\end{array}\right)\left(\begin{array}{c}
d_{x}^{k, j} \\
d_{\lambda}^{k, j} \\
d_{z_{\ell}}^{k, j} \\
d_{z_{u}}^{k, j}
\end{array}\right)=-\left(\begin{array}{c}
\nabla f\left(x^{k, j}\right)+A^{T} \lambda^{k, j}-z_{\ell}^{k, j}+z_{u}^{k, j} \\
0 \\
L_{k, j} z_{\ell}^{k, j}-\mu_{k} e \\
U_{k, j} z_{u}^{k, j}-\mu_{k} e
\end{array}\right)
$$


O sistema linear (2.12) é chamado primal-dual [21]. Seus dois últimos blocos de equações implicam que

$$
\begin{aligned}
Z_{k, j}^{\ell} d_{x}^{k, j}+L_{k, j} d_{z_{\ell}}^{k, j} & =-L_{k, j} z_{\ell}^{k, j}+\mu_{k} e \\
-Z_{k, j}^{u} d_{x}^{k, j}+U_{k, j} d_{z_{u}}^{k, j} & =-U_{k, j} z_{u}^{k, j}+\mu_{k} e .
\end{aligned}
$$

Colocando em evidencia $d_{z_{\ell}}^{k, j}$ e $d_{z_{u}}^{k, j}$, temos que

$$
\begin{aligned}
& d_{z_{\ell}}^{k, j}=L_{k, j}^{\dagger}\left(-L_{k, j} Z_{k, j}^{\ell} e+\mu_{k} e-Z_{k, j}^{\ell} d_{x}^{k, j}\right)=-z_{\ell}^{k, j}+\mu_{k} L_{k, j}^{\dagger} e-L_{k, j}^{\dagger} Z_{k, j}^{\ell} d_{x}^{k, j} \\
& d_{z_{u}}^{k, j}=U_{k, j}^{\dagger}\left(-U_{k, j} Z_{k, j}^{u} e+\mu_{k} e+Z_{k, j}^{u} d_{x}^{k, j}\right)=-z_{u}^{k, j}+\mu_{k} U_{k, j}^{\dagger} e+U_{k, j}^{\dagger} Z_{k, j}^{u} d_{x}^{k, j} .
\end{aligned}
$$

Substituindo $d_{z_{\ell}}^{k, j}$ e $d_{z_{u}}^{k, j}$ no primeiro bloco de equações do sistema (2.12) obtemos o sistema de dimensão $n+m$ dado por

$$
\left(\begin{array}{cc}
H_{k, j} & A^{T} \\
A & 0
\end{array}\right)\left(\begin{array}{c}
d_{x}^{k, j} \\
d_{\lambda}^{k, j}
\end{array}\right)=-\left(\begin{array}{c}
\nabla f\left(x^{k, j}\right)+A^{T} \lambda^{k, j}-\mu_{k} L_{k, j}^{\dagger} e+\mu_{k} U_{k, j}^{\dagger} e \\
0
\end{array}\right)
$$

onde

$$
H_{k, j}=\nabla^{2} f\left(x^{k, j}\right)+L_{k, j}^{\dagger} Z_{k, j}^{\ell}+U_{k, j}^{\dagger} Z_{k, j}^{u} .
$$

A resolução do sistema (2.14) requer alguns cuidados, que discutiremos na Seção 2.3. Uma vez calculados $d_{x}^{k, j}$ e $d_{\lambda}^{k, j}$, encontramos $d_{z_{\ell}}^{k, j}$ e $d_{z_{u}}^{k, j}$ usando (2.13). Tendo calculado as direções, definimos

$$
\begin{aligned}
x^{k, j+1} & =x^{k, j}+\alpha_{k, j} d_{x}^{k, j} \\
\lambda^{k, j+1} & =\lambda^{k, j}+d_{\lambda}^{k, j} \\
\bar{z}_{\ell}^{k, j+1} & =z_{\ell}^{k, j}+\alpha_{k, j}^{z_{\ell}} d_{z_{\ell}}^{k, j} \\
\bar{z}^{u, j+1} & =z_{u}^{k, j}+\alpha_{k, j}^{z_{u}} d_{z_{u}}^{k, j},
\end{aligned}
$$

de forma que $\alpha_{k, j}, \alpha_{k, j}^{z_{\ell}}, \alpha_{k, j}^{z_{u}} \in(0,1]$ determinam os tamanhos de cada passo a serem dados em (2.16). Dado que $\ell<x^{k, j}<u,\left[z_{\ell}^{k, j}\right]_{i}>0$ para cada $i \in \mathcal{I}_{\ell}$ e $\left[z_{u}^{k, j}\right]_{i}>0$ para cada $i \in \mathcal{I}_{u}$, cuidamos para que o passo tomado conserve tais propriedades no novo ponto. Para isto, definimos uma fração à fronteira

$$
\tau=\max \left\{\tau_{\min }, 1-\mu_{k}\right\}
$$

onde $\tau_{\min } \in(0,1)$ é o menor valor para esta fração, e calculamos

$$
\begin{aligned}
\alpha_{k, j}^{\ell} & =\max \left\{\alpha \in(0,1]:\left(x_{i}^{k, j}+\alpha\left[d_{x}^{k, j}\right]_{i}\right)-\ell_{i} \geq(1-\tau)\left(x_{i}^{k, j}-\ell_{i}\right)\right\}, \text { para } i \in \mathcal{I}_{\ell} \cap \mathcal{D}_{-}^{k, j} \\
\alpha_{k, j}^{u} & =\max \left\{\alpha \in(0,1]: u_{i}-\left(x_{i}^{k, j}+\alpha\left[d_{x}^{k, j}\right]_{i}\right) \geq(1-\tau)\left(u_{i}-x_{i}^{k, j}\right)\right\}, \text { para } i \in \mathcal{I}_{u} \cap \mathcal{D}_{+}^{k, j} \\
\alpha_{k, j}^{z \ell} & =\max \left\{\alpha \in(0,1]:\left[z_{\ell}^{k, j}\right]_{i}+\alpha\left[d_{z_{\ell}}^{k, j}\right]_{i} \geq(1-\tau)\left[z_{\ell}^{k, j}\right]_{i}\right\}, \text { para } i \in \mathcal{I}_{\ell} \cap \mathcal{D}_{+}^{k, j} \\
\alpha_{k, j}^{z_{u}} & =\max \left\{\alpha \in(0,1]:\left[z_{u}^{k, j}\right]_{i}+\alpha\left[d_{z_{u}}^{k, j}\right]_{i} \geq(1-\tau)\left[z_{u}^{k, j}\right]_{i}\right\}, \text { para } i \in \mathcal{I}_{u} \cap \mathcal{D}_{-}^{k, j},
\end{aligned}
$$

onde $\mathcal{D}_{-}^{k, j}=\left\{i:\left[d_{x}^{k, j}\right]_{i}<0\right\}$ e $\mathcal{D}_{+}^{k, j}=\left\{i:\left[d_{x}^{k, j}\right]_{i}>0\right\}$, obtendo, por fim,

$$
\alpha_{k, j}^{\max }=\min \left\{\alpha_{k, j}^{\ell}, \alpha_{k, j}^{u}\right\}
$$

Depois, fazemos backtracking para obter $\alpha_{k, j} \in\left(0, \alpha_{k, j}^{\max }\right]$ de forma que $x^{k, j}+\alpha_{k, j} d_{x}^{k, j}$ satisfaça (2.11).

Por fim, o último cuidado é atentar para que $z_{\ell}^{k, j+1}$ e $z_{u}^{k, j+1}$ conservem, aproximadamente, a relação com $x^{k, j+1}$ dada em (2.7). Embora não sejam usadas na prática, são essas equações que 
garantem que a matriz do noroeste do sistema (2.14) é uma aproximação para a matriz Hessiana da função barreira (2.9). Assim sendo, tomamos

$$
\left[z_{\ell}^{k, j+1}\right]_{i}=\left\{\begin{array}{cl}
\max \left\{\min \left\{\left[\bar{z}_{\ell}^{k, j+1}\right]_{i}, \kappa_{z}\left(\frac{\mu_{k}}{x_{i}^{k, j+1}-\ell_{i}}\right)\right\}, \frac{1}{\kappa_{z}}\left(\frac{\mu_{k}}{x_{i}^{k, j+1}-\ell_{i}}\right)\right\}, & \text { se } i \in \mathcal{I}_{\ell} \\
0, & \text { caso contrário }
\end{array}\right.
$$

e

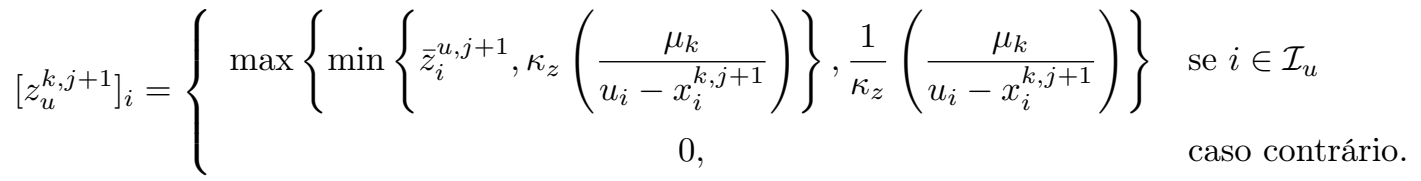

com $i=1, \ldots, n$ e $\kappa_{z} \geq 1$ uma constante definida. Com isso, teremos que

$$
\begin{aligned}
{\left[z_{\ell}^{k, j+1}\right]_{i} } & \in\left[\frac{1}{\kappa_{z}}\left(\frac{\mu_{k}}{x_{i}^{k, j+1}-\ell_{i}}\right), \kappa_{z}\left(\frac{\mu_{k}}{x_{i}^{k, j+1}-\ell_{i}}\right)\right], \text { para } i \in \mathcal{I}_{\ell} \mathrm{e} \\
{\left[z_{u}^{k, j+1}\right]_{i} } & \in\left[\frac{1}{\kappa_{z}}\left(\frac{\mu_{k}}{u_{i}-x_{i}^{k, j+1}}\right), \kappa_{z}\left(\frac{\mu_{k}}{u_{i}-x_{i}^{k, j+1}}\right)\right], \text { para } i \in \mathcal{I}_{u},
\end{aligned}
$$

ou seja, que $z_{\ell}^{k, j+1}$ e $z_{u}^{k, j+1}$ satisfarão a relação (2.7) de acordo com uma tolerância $\kappa_{z}$. Neste contexto, é interessante tomar valores altos para $\kappa_{z}$, de tal maneira que a correção não influa na convergência do algoritmo. Diante disso, consideraremos $\kappa_{z}=10^{10}$.

Para cada subproblema (2.10) consideraremos que um ponto $\left(x^{k, j}, \lambda^{k, j}, z_{\ell}^{k, j}, z_{u}^{k, j}\right)$ é uma solução aproximada quando

$$
E_{\mu_{k}}\left(x^{k, j}, \lambda^{k, j}, z_{\ell}^{k, j}, z_{u}^{k, j}\right) \leq \kappa_{\varepsilon} \mu_{k}
$$

sendo

$$
E_{\mu}\left(x, \lambda, z_{\ell}, z_{u}\right)=\left\|\left(\begin{array}{c}
\nabla f(x)+A^{T} \lambda-z_{\ell}+z_{u} \\
L z_{\ell}-\mu e \\
U z_{u}-\mu e
\end{array}\right)\right\|_{\infty}
$$

e $\kappa_{\varepsilon}>0$. Desse modo, (2.23) implica que as equações (2.8a), (2.8c) e (2.8d) são aproximadamente satisfeitas e, pela definição do método, $A x^{k, j}=b$, o que satisfaz (2.8b), logo $\left(x^{k, j}, \lambda^{k, j}, z_{\ell}^{k, j}, z_{u}^{k, j}\right)$ satisfaz aproximadamente as condições de otimalidade de primeira ordem (2.8) do subproblema (2.10).

Tendo resolvido um subproblema, obtemos um novo parâmetro de barreira

$$
\mu_{k+1}=\min \left\{\kappa_{\mu} \mu_{k}, \mu_{k}^{\theta_{\mu}}\right\}
$$

onde $\kappa_{\mu} \in(0,1)$ e $\theta_{\mu} \in(1,2)$. Deste modo, o parâmetro de barreira pode eventualmente convergir a zero superlinearmente.

\subsection{Resolução do sistema primal-dual}

Nesta seção, discutiremos alguns detalhes sobre a resolução do sistema primal-dual reduzido, apresentado na equação (2.14). Em primeiro lugar, é necessário garantir que (2.14) possua solução. A priori, nada garante que (2.14) possui solução, dado que a matriz do sistema pode ser singular. Queremos também cuidar para que a direção $d_{x}^{k, j}$ calculada seja uma direção de descida para $\varphi_{\mu_{k}}(\cdot)$, definida em (2.9), a partir de $x^{k, j}$. À luz disto, começaremos com a seguinte definição. 
Definição 2. Seja K uma matriz simétrica qualquer. Sua inércia, denotada por In $(K)$, é a tripla $\left(a_{+}, a_{-}, a_{0}\right)$ onde $a_{+}, a_{-}$e $a_{0}$ denotam o número de autovalores positivos, negativos e nulos da matriz $K$, respectivamente.

Para o desenvolvimento das ideias desta seção, consideraremos uma forma geral do sistema (2.14) dada por

$$
\left(\begin{array}{cc}
\hat{H} & A^{T} \\
A & 0
\end{array}\right)\left(\begin{array}{c}
d_{x} \\
d_{\lambda}
\end{array}\right)=-\left(\begin{array}{c}
\nabla f(x)+A^{T} \lambda-\mu_{k} L^{\dagger} e+\mu_{k} U^{\dagger} e \\
0
\end{array}\right)
$$

onde $A \in \mathbb{R}^{m \times n}$ é uma matriz com posto linha completo e $\hat{H} \in \mathbb{R}^{n \times n}$.

A seguinte proposição, adaptada de [21, Teorema 16.3] e cuja demonstração pode ser encontrada com detalhes em [16, Lema 3.4], nos dá um resultado interessante para obter o que queremos.

Proposição 3. Consideremos a forma geral do sistema primal-dual reduzido (2.14) dada em (2.26), e suponhamos que A possui posto completo. Então,

$$
\operatorname{In}\left(\begin{array}{cc}
\hat{H} & A^{T} \\
A & 0
\end{array}\right)=\operatorname{In}\left(W^{T} \hat{H} W\right)+(m, m, 0)
$$

onde $W \in \mathbb{R}^{n \times(n-m)}$ é uma matriz cujas colunas formam uma base para o núcleo da matriz $A$.

A Proposição 3 nos permite concluir que a matriz do sistema (2.14) será não singular desde que a matriz do noroeste do sistema projetada no núcleo da $A$ não possua autovalores nulos. Consideremos ainda o seguinte resultado.

Lema 4. Consideremos a forma geral do sistema primal-dual reduzido (2.14) dada em (2.26). Se $\hat{H}$ for definida positiva no núcleo da matriz $A$ e $d_{x}$, obtida como solução do sistema (2.26), for não nula, então $d_{x}$ será uma direção de descida para $\varphi_{\mu_{k}}(\cdot)$ a partir de $x$.

Demonstração. Pela primeira equação do sistema (2.26), temos que

$$
\hat{H} d_{x}+A^{T} d_{\lambda}=-\nabla f(x)-A^{T} \lambda+\mu_{k} L^{\dagger} e-\mu_{k} U^{\dagger} e .
$$

Como $\nabla \varphi_{\mu_{k}}(x)=\nabla f(x)-\mu_{k} L^{\dagger} e+\mu_{k} U^{\dagger} e$, de (2.27) segue que

$$
\hat{H} d_{x}+A^{T} d_{\lambda}=-\nabla \varphi_{\mu_{k}}(x)-A^{T} \lambda .
$$

A segunda equação do sistema (2.26), por sua vez, implica que $d_{x}$ pertence ao núcleo da matriz $A$. Em vista disto, pré-multiplicando $(2.28)$ por $-\left(d_{x}\right)^{T}$, vem que

$$
\nabla \varphi_{\mu_{k}}(x)^{T} d_{x}=-\left(d_{x}\right)^{T} \hat{H} d_{x} .
$$

Logo, se $\hat{H}$ for definida positiva no núcleo da $A$, então $\left(d_{x}\right)^{T} \hat{H} d_{x}>0$ sempre que $d_{x} \neq 0$, o que, junto com (2.29), implica que $d_{x}$ é um direção de descida para $\varphi_{\mu_{k}}(\cdot)$ a partir de $x$.

A Proposição 3 e o Lema 4 , juntos, implicam que se $\hat{H}$ for definida positiva no núcleo da matriz $A$, então qualquer sistema da forma (2.26) admite solução única $d_{x}$, e $d_{x}$ é uma direção de descida para $\varphi_{\mu_{k}}(\cdot)$ a partir de $x$ sempre que for não nula. Mais ainda, a Proposição 3 implica que a inércia da matriz do sistema $(2.26)$ será $(n, m, 0)$ se, e somente se, $\hat{H}$ for positiva definida no núcleo da $A$. Assim sendo, caso a matriz do sistema (2.14) não possua inércia $(n, m, 0)$, repetimos a fatoração da matriz

$$
M_{k, j}=\left(\begin{array}{cc}
H_{k, j}+\xi_{k, j} I & A^{T} \\
A & 0
\end{array}\right)
$$


até encontrar $\xi_{k, j}>0$ tal que $M_{k, j}$ possua a inércia desejada. Com isso, resolvemos o sistema (2.14) perturbado

$$
M_{k, j}\left(\begin{array}{c}
d_{x}^{k, j} \\
d_{\lambda}^{k, j}
\end{array}\right)=-\left(\begin{array}{c}
\nabla \varphi_{\mu_{k}}\left(x^{k, j}\right)+A^{T} \lambda_{k, j} \\
0
\end{array}\right) .
$$

\subsection{Modelo algorítmico para a resolução do subproblema}

Nesta seção, apresentaremos o modelo algorítmico que descreve o método para a resolução do subproblema (2.10). A fim de facilitar a leitura, apresentamos um glossário da notação usada no algoritmo.

\begin{tabular}{|c|c|}
\hline \multicolumn{2}{|c|}{ Notação } \\
\hline$n$ : quantidade de variáveis & $\kappa_{\mu}:$ fator para cálculo de $\mu$ \\
\hline$m$ : quantidade de restrições $A x=b$ & $\theta_{\mu}:$ fator para cálculo de $\mu$ \\
\hline$k$ : contador de iterações externas & $\xi_{k, j}:$ termo de correção da inércia do sistema (2.31) \\
\hline$j:$ contador de iterações internas & $\kappa_{z}$ : termo de ajuste das variáveis $z_{\ell}$ e $z_{u}$ \\
\hline$f:$ função objetivo & $\gamma:$ constante de Armijo \\
\hline$\varphi_{\mu}:$ função barreira $(2.9)$ & $d_{x}^{k, j}:$ direção de busca em $x^{k, j}$ \\
\hline$E_{\mu}:$ erro de otimalidade $(2.24)$ & $d_{\lambda}^{k, j}:$ direção de busca em $\lambda^{k, j}$ \\
\hline$x^{k, j}$ : iterando primal & $d_{z_{\ell}}^{k, j}:$ direção de busca em $z_{\ell}^{k, j}$ \\
\hline$\lambda^{k, j}:$ iterando dual & $d_{z_{u}}^{k, j}:$ direção de busca em $z_{u}^{k, j}$ \\
\hline$z_{\ell}^{k, j}:$ iterando dual & $\alpha_{k, j}^{\ell}:$ passo máximo de $x^{k, j}$ inferiormente \\
\hline$z_{u}^{k, j}:$ iterando dual & $\alpha_{k, j}^{u, j}:$ passo máximo de $x^{k, j}$ superiormente \\
\hline$\varepsilon_{\text {tol }}$ : tolerância de otimalidade & $\alpha_{k, j}^{\max }:$ passo máximo de $x^{k, j}$ \\
\hline$\kappa_{\varepsilon}$ : fator de tol. de otimalidade do subproblema & $\alpha_{k, j}^{z \ell}:$ passo máximo de $z_{\ell}^{k, j}$ \\
\hline$\mu_{k}$ : parâmetro barreira & $\alpha_{k, j}^{z_{u}^{u}}:$ passo máximo de $z_{u}^{k, j}$ \\
\hline$L_{k, j}:$ matriz diagonal $(2.6) \operatorname{com} x=x^{k, j}$ & $\mathcal{I}_{\ell}:\left\{i: \ell_{i}>-\infty\right\}$ \\
\hline$U_{k, j}:$ matriz diagonal (2.6) com $x=x^{k, j}$ & $\mathcal{I}_{u}:\left\{i: u_{i}<\infty\right\}$ \\
\hline$Z_{k, j}^{\ell}: \operatorname{diag}\left(z_{\ell}^{k, j}\right)$ & $\mathcal{D}_{+}^{k, j}:\left\{i:\left[d_{x}^{k, j}\right]_{i}>0\right\}$ \\
\hline$Z_{k, j}^{u}: \operatorname{diag}\left(z_{u}^{k, j}\right)$ & $\mathcal{D}_{-}^{k, j}:\left\{i:\left[d_{x}^{k, j}\right]_{i}<0\right\}$ \\
\hline
\end{tabular}

Algoritmo 1. Método de pontos interiores - Algoritmo interno.

Entrada: $x^{k, 0} \in \mathcal{F}_{0}, \lambda^{k, 0} \in \mathbb{R}^{m}, z_{\ell}^{k, 0} \in \mathbb{R}^{n}$ e $z_{u}^{k, 0} \in \mathbb{R}^{n}$ tais que $\left[z_{\ell}^{k, 0}\right]_{i}>0$ para $i \in \mathcal{I}_{\ell}$ e $\left[z_{\ell}^{k, 0}\right]_{i}=0$ caso contrário, e $\left[z_{u}^{k, 0}\right]_{i}>0$ para $i \in \mathcal{I}_{u}$ e $\left[z_{u}^{k, 0}\right]_{i}=0$ caso contrário, $A \in \mathbb{R}^{m \times n}$ com posto linha completo e $\mu_{k}>0$; constantes $\kappa_{\varepsilon}>0, \gamma \in(0,1), \tau_{\min }>0, \kappa_{z} \geq 1 ; \xi_{0,-1} \leftarrow 0$ e $j \leftarrow 0$.

Passo 1. Se $E_{\mu_{k}}\left(x^{k, j}, \lambda^{k, j}, z_{\ell}^{k, j}, z_{u}^{k, j}\right) \leq \kappa_{\varepsilon} \mu_{k}$, então pare e retorne $\left(x^{k, j}, \lambda^{k, j}, z_{\ell}^{k, j}, z_{u}^{k, j}\right)$ como ponto aproximadamente estacionário.

Passo 2. Utilizando $\xi_{k, j-1}$, calcule $\xi_{k, j} \geq 0$ tal que $\operatorname{In}\left(M_{k, j}\right)=(n, m, 0)$, onde

$$
M_{k, j}=\left(\begin{array}{cc}
\nabla^{2} f\left(x^{k, j}\right)+L_{k, j}^{\dagger} Z_{k, j}^{L}+U_{k, j}^{\dagger} Z_{k, j}^{U}+\xi_{k, j} I & A^{T} \\
A & 0
\end{array}\right) .
$$

Passo 3. Encontre $d_{x}^{k, j}$ e $d_{\lambda}^{k, j}$ solução do sistema

$$
M_{k, j}\left(\begin{array}{c}
d_{x}^{k, j} \\
d_{\lambda}^{k, j}
\end{array}\right)=-\left(\begin{array}{c}
\nabla \varphi_{\mu_{k}}\left(x^{k, j}\right)+A^{T} \lambda^{k, j} \\
0
\end{array}\right) .
$$


Passo 4. Calcule

$$
\begin{aligned}
& d_{z_{\ell}}^{k, j}=-z_{\ell}^{k, j}+\mu L_{k, j}^{\dagger} e-L_{k, j}^{\dagger} Z_{k, j}^{L} d_{x}^{k, j} \mathrm{e} \\
& d_{z_{u}}^{k, j}=-z_{u}^{k, j}+\mu U_{k, j}^{\dagger} e+U_{k, j}^{\dagger} Z_{k, j}^{U} d_{x}^{k, j}
\end{aligned}
$$

Passo 5. Calcule $\tau=\max \left\{\tau_{\min }, 1-\mu_{k}\right\}$ e

$$
\begin{aligned}
& \alpha_{k, j}^{\ell}=\max \left\{\alpha \in(0,1]:\left(\left[x^{k, j}\right]_{i}+\alpha\left[d_{x}^{k, j}\right]_{i}\right)-\ell \geq(1-\tau)\left(\left[x^{k, j}\right]_{i}-\ell_{i}\right)\right\} \text {, para } i \in \mathcal{I}_{\ell} \cap \mathcal{D}_{-}^{k, j}, \\
& \alpha_{k, j}^{u}=\max \left\{\alpha \in(0,1]: u_{i}-\left(\left[x^{k, j}\right]_{i}+\alpha\left[d_{x}^{k, j}\right]_{i}\right) \geq(1-\tau)\left(u_{i}-\left[x^{k, j}\right]_{i}\right)\right\} \text {, para } i \in \mathcal{I}_{u} \cap \mathcal{D}_{+}^{k, j} \text {, } \\
& \alpha_{k, j}^{z_{\ell}}=\max \left\{\alpha \in(0,1]:\left[z_{\ell}^{k, j}\right]_{i}+\alpha\left[d_{z_{\ell}}^{k, j}\right]_{i} \geq(1-\tau)\left[z_{\ell}^{k, j}\right]_{i}\right\} \text {, para } i \in \mathcal{I}_{\ell} \cap \mathcal{D}_{+}^{k, j} \text {, } \\
& \alpha_{k, j}^{z_{u}}=\max \left\{\alpha \in(0,1]:\left[z_{u}^{k, j}\right]_{i}+\alpha\left[d_{z_{u}}^{k, j}\right]_{i} \geq(1-\tau)\left[z_{u}^{k, j}\right]_{i}\right\} \text {, para } i \in \mathcal{I}_{u} \cap \mathcal{D}_{-}^{k, j} \\
& \text { e } \\
& \alpha_{k, j}^{\max }=\min \left\{\alpha_{k, j}^{\ell}, \alpha_{k, j}^{u}\right\}
\end{aligned}
$$

Passo 6. Faça $\alpha_{k, j} \leftarrow \alpha_{k, j}^{\max }$. Se

$$
\max _{1 \leq i \leq n}\left\{\frac{\left|\left(d_{x}^{k, j}\right)_{i}\right|}{\left(1+\left|x_{i}^{k, j}\right|\right)}\right\} \not \leq 10 \varepsilon_{\text {mach }},
$$

então, enquanto $\varphi_{\mu_{k}}\left(x^{k, j}+\alpha_{k, j} d_{x}^{k, j}\right)>\varphi_{\mu_{k}}\left(x^{k, j}\right)+\gamma \alpha_{k, j} \nabla \varphi_{\mu_{k}}\left(x^{k, j}\right)^{T} d_{x}^{k, j}$, faça $\alpha_{k, j} \leftarrow \frac{1}{2} \alpha_{k, j}$.

Passo 7. Faça $x^{k, j+1}=x^{k, j}+\alpha_{k, j} d_{x}^{k, j}$ e $\lambda^{k, j+1}=\lambda^{k, j}+d_{\lambda}^{k, j}$.

Passo 8. Faça $\bar{z}_{\ell}^{k, j+1}=z_{\ell}^{k, j}+\alpha_{k, j}^{z_{\ell}} d_{z_{\ell}}^{k, j}, \bar{z}_{u}^{k, j+1}=z_{u}^{k, j}+\alpha_{k, j}^{z_{u}} d_{z_{u}}^{k, j}$,

$$
\left[z_{\ell}^{k, j+1}\right]_{i}=\left\{\begin{aligned}
\max \left\{\min \left\{\left[\bar{z}_{\ell}^{k, j+1}\right]_{i}, \kappa_{z}\left(\frac{\mu_{k}}{\left[x^{k, j+1}\right]_{i}-\ell_{i}}\right)\right\}, \frac{1}{\kappa_{z}}\left(\frac{\mu_{k}}{\left[x^{k, j+1}\right]_{i}-\ell_{i}}\right)\right\}, & \text { se } i \in \mathcal{I}_{\ell}, \\
0, & \text { caso contrário }
\end{aligned}\right.
$$

e

$$
\left[z_{u}^{k, j+1}\right]_{i}=\left\{\begin{aligned}
\max \left\{\min \left\{\left[\bar{z}_{u}^{k, j+1}\right]_{i}, \kappa_{z}\left(\frac{\mu_{k}}{u_{i}-\left[x^{k, j+1}\right]_{i}}\right)\right\}, \frac{1}{\kappa_{z}}\left(\frac{\mu_{k}}{u_{i}-\left[x^{k, j+1}\right]_{i}}\right)\right\}, & \text { se } i \in \mathcal{I}_{u}, \\
0, & \text { caso contrário. }
\end{aligned}\right.
$$

Passo 9. Faça $j \leftarrow j+1$ e vá para o Passo 1 .

\subsubsection{Boa definição}

Queremos, nesta seção, mostrar que o Algoritmo 1 está bem definido. Em primeiro lugar, pela Hipótese 1 , existe um ponto $x^{k, 0} \in \mathcal{F}_{0}$. O resultado a seguir diz respeito ao cálculo de $\xi_{k, j}$ no Passo 2, que será computado usando o Algoritmo 3.

Lema 5. Suponha que estamos no Passo 2 do Algoritmo 1. Então, existe $\xi_{k, j} \geq 0$ suficientemente grande tal que $\operatorname{In}\left(M_{k, j}\right)=(n, m, 0)$.

Demonstração. Seja $\tilde{H}_{\xi_{k, j}}=\nabla^{2} f\left(x^{k, j}\right)+L_{k, j}^{\dagger} Z_{k, j}^{L}+U_{k, j}^{\dagger} Z_{k, j}^{U}+\xi_{k, j} I$ e $W \in \mathbb{R}^{n \times n-m}$ uma matriz cujas colunas formam uma base para o núcleo da $A$. Notemos que, se $\tilde{H}_{\xi_{k, j}}$ for definida positiva, então $W^{T} \tilde{H}_{\xi_{k, j}} W$ também é. De fato, para todo $\omega \in \mathbb{R}^{n-m}$ não nulo, temos que

$$
\omega^{T} W \tilde{H}_{\xi_{k, j}} W \omega=y^{T} \tilde{H}_{\xi_{k, j}} y>0,
$$


haja visto que $y=W \omega \neq 0$ pois $\omega \neq 0$ e as colunas de $W$ são linearmente independentes.

Logo, se, por um lado, $\tilde{H}_{0}$ for positiva definida, então $W^{T} \tilde{H}_{0} W$ também é e, pela Proposição 3 aplicada ao sistema $(2.31)$, vem que $\operatorname{In}\left(M_{k, j}\right)=(n, m, 0)$ para $\xi_{k, j}=0$. Se, por outro lado, $\tilde{H}_{0}$ não for positiva definida, consideremos $\lambda_{1} \leq \lambda_{2} \leq \ldots \leq \lambda_{n}$ os autovalores de $\tilde{H}_{0}$, com $\lambda_{1} \leq 0$. Então a matriz $\tilde{H}_{\left|\lambda_{1}\right|+\epsilon}$, para $\epsilon>0$, possuirá autovalores

$$
\epsilon \leq \lambda_{2}+\left|\lambda_{1}\right|+\epsilon \leq \ldots \leq \lambda_{n}+\left|\lambda_{1}\right|+\epsilon,
$$

donde segue que $\tilde{H}_{\left|\lambda_{1}\right|+\epsilon}$ é uma matriz positiva definida e, portanto, $W^{T} \tilde{H}_{\left|\lambda_{1}\right|+\epsilon} W$ também é. Deste modo, pela Proposição 3 aplicada ao sistema (2.31), $\operatorname{In}\left(M_{k, j}\right)=(n, m, 0)$ para $\xi_{k, j} \geq\left|\lambda_{1}\right|+\epsilon$.

O resultado a seguir diz respeito ao cálculo das direções de busca.

Lema 6. Suponha que estamos no Passo 3 do Algoritmo 1. Então, é possível calcular as direções de busca $d_{x}^{k, j}$ e $d_{\lambda}^{k, j}$ como solução do sistema (2.31) e, se $d_{x}^{k, j} \neq 0$, então $d_{x}^{k, j}$ será uma direção de descida para $\varphi_{\mu_{k}}(\cdot)$ a partir de $x^{k, j}$.

Demonstração. Pelo Lema 5, é possível encontrar $\xi_{k, j}$ tal que $\operatorname{In}\left(M_{k, j}\right)=(n, m, 0)$. Portanto, $M_{k, j}$ é uma matriz não singular, e o sistema (2.31) possui solução única. Mais ainda, pelo Lema 4 aplicado ao sistema $(2.31)$, como $\operatorname{In}\left(M_{k, j}\right)=(n, m, 0)$, temos que se $d_{x}^{k, j} \neq 0$, então $d_{x}^{k, j}$ será uma direção de descida para $\varphi_{\mu_{k}}(\cdot)$ a partir de $x^{k, j}$.

Por fim, mostramos no Lema 7 que o procedimento de busca linear do Passo 6 do Algoritmo 1 está bem definido.

Lema 7. Suponha que estamos no Passo 6 do Algoritmo 1. Então, existe $\bar{\alpha} \in \mathbb{R}$ tal que para todo $\alpha_{k, j} \in(0, \bar{\alpha}]$ a condição de Armijo (2.11) se cumpre.

Demonstração. Se $d_{x}^{k, j}=0$, então naturalmente a condição de Armijo (2.11) valerá para todo $\alpha_{k, j} \in\left(0, \alpha_{k, j}^{\max }\right]$. Suponhamos então que $d_{x}^{k, j} \neq 0$. Pelo Lema 6 , vem que $\nabla \varphi_{\mu_{k}}\left(x^{k, j}\right)^{T} d_{x}^{k, j}<0$. Como $\varphi_{\mu_{k}}(\cdot)$ é uma função diferenciável, temos que sua derivada direcional ao longo de $d_{x}^{k, j}$ é dada por

Assim sendo,

$$
\lim _{\alpha \rightarrow 0^{+}} \frac{\varphi_{\mu_{k}}\left(x^{k, j}+\alpha d_{x}^{k, j}\right)-\varphi_{\mu_{k}}\left(x^{k, j}\right)}{\alpha}=\nabla \varphi_{\mu_{k}}\left(x^{k, j}\right)^{T} d_{x}^{k, j}<0 .
$$

$$
\lim _{\alpha \rightarrow 0^{+}} \frac{\varphi_{\mu_{k}}\left(x^{k, j}+\alpha d_{x}^{k, j}\right)-\varphi_{\mu_{k}}\left(x^{k, j}\right)}{\alpha \nabla \varphi_{\mu_{k}}\left(x^{k, j}\right)^{T} d_{x}^{k, j}}=1 .
$$

Tomando $\gamma \in(0,1)$, existe $\bar{\alpha}$ suficientemente pequeno tal que, para todo $\alpha \in(0, \bar{\alpha})$,

$$
\frac{\varphi_{\mu_{k}}\left(x^{k, j}+\alpha d_{x}^{k, j}\right)-\varphi_{\mu_{k}}\left(x^{k, j}\right)}{\alpha \nabla \varphi_{\mu_{k}}\left(x^{k, j}\right)^{T} d_{x}^{k, j}} \geq \gamma .
$$

Por conseguinte, como $\nabla \varphi_{\mu_{k}}\left(x^{k, j}\right)^{T} d_{x}^{k, j}<0$, vem que

$$
\varphi_{\mu_{k}}\left(x^{k, j}+\alpha d_{x}^{k, j}\right) \leq \varphi_{\mu_{k}}\left(x^{k, j}\right)+\gamma \alpha \nabla \varphi_{\mu_{k}}\left(x^{k, j}\right)^{T} d_{x}^{k, j},
$$

para todo $\alpha \in(0, \bar{\alpha})$.

Por conseguinte, o Algoritmo 1 para apenas no Passo 1, em que o ponto corrente satisfaz, aproximadamente, as condições de otimalidade do problema (2.10). Estabelecemos então a boa definição do Algoritmo 1 na seguinte proposição.

Proposição 8. Se as Hipóteses 1 e 2 valem, então o Algoritmo 1 está bem definido. 


\subsubsection{Análise da convergência global}

A análise da convergência global possui forte embasamento no trabalho de Chen e Goldfarb [12]. Para mostrar as propriedades de convergência global do Algoritmo 1, faremos as seguintes hipóteses adicionais.

Hipótese 3. A sequência $\left\{x^{k, j}\right\}$ gerada pelo Algoritmo 1 é limitada, para todo $k$.

Hipótese 4. As matrizes $\bar{H}_{k, j}=H_{k, j}+\xi_{k, j} I$ satisfazem

$$
d^{T} \bar{H}_{k, j} d \geq \sigma\|d\|^{2}
$$

para todo $d \neq 0$ tal que $A d=0$, para algum $\sigma>0$ e para todo $k$ e todo $j$.

Embora a Hipótese 3 seja uma conjectura feita sobre a sequência gerada pelo método, ela será satisfeita sempre que existirem restrições de caixas para todas as variáveis, isto é, quando $-\infty<\ell_{i} \leq u_{i}<+\infty$, para todo $i=1, \ldots, n$, ou sempre que o conjunto $\left\{x \in \mathcal{F} \mid f(x) \leq f\left(x^{0}\right)\right\}$ for limitado, onde $x^{0} \in \mathcal{F}_{0}$ é um ponto inicial estritamente viável. A Hipótese 4, por sua vez, estabelece que as matrizes $\left\{\bar{H}_{k, j}\right\}$ devem ser uniformemente positivas definidas no núcleo da matriz A. Em comparação com a exigência semelhante no trabalho de Chen e Goldfarb [12, Condição C-5], esta hipótese é mais fraca, visto que o Lema 5 garante que é possível encontrar $\xi_{k, j}$ no Passo 2 do Algoritmo 1 tal que $\bar{H}_{k, j}$ é definida positiva. Deste modo, a Hipótese 4 deve ser assegurada pela natureza numérica do método de correção de inércia usado no Algoritmo 3.

A boa definição estabelecida na Proposição 8 implica que o Algoritmo 1 para apenas no Passo 1 quando o ponto corrente satisfizer, aproximadamente, as condições de otimalidade de primeira ordem do subproblema (2.10). Nos resultados que seguem, analisamos o comportamento do Algoritmo 1 quando não para, isto é, quando gera sequências infinitas.

Lema 9. Suponhamos que as Hipóteses 1, 2 e 3 valem e seja $\left\{x^{k, j+1}, \lambda^{k, j+1}, z_{\ell}^{k, j+1}, z_{u}^{k, j+1}\right\}$ a sequência gerada pelo Algoritmo 1. Então, existe $\delta>0$ tal que

I. para todo $j$ vale

(a) $\ell_{i}+\delta \leq x_{i}^{k, j+1}$, para todo $i \in \mathcal{I}_{\ell} e$

(b) $x_{i}^{k, j+1} \leq u_{i}-\delta$, para todo $i \in \mathcal{I}_{u}$;

II. para todo $j$ vale

(a) $\left[z_{\ell}^{k, j+1}\right]_{i} \in \frac{\mu_{k}}{\delta}\left[\frac{1}{\kappa_{z}}, \kappa_{z}\right]$, para todo $i \in \mathcal{I}_{\ell} e$

(b) $\left[z_{u}^{k, j+1}\right]_{i} \in \frac{\mu_{k}}{\delta}\left[\frac{1}{\kappa_{z}}, \kappa_{z}\right]$, para todo $i \in \mathcal{I}_{u}$.

Demonstração. Para demonstrar a parte (I), suponhamos, por contradição, que existe um conjunto infinito $\mathcal{J} \subset \mathbb{\infty}$ e um índice $\hat{i} \in \mathcal{I}_{\ell}$ tais que

$$
\lim _{j \in \mathcal{J}} x_{\hat{i}}^{k, j+1}=\ell_{\hat{i}}
$$

Pelo Passo 1, temos que $E_{\mu_{k}}\left(x^{k, j+1}, \lambda^{k, j+1}, z_{\ell}^{k, j+1}, z_{u}^{k, j+1}\right)>\kappa_{\varepsilon} \mu_{k}$ e, pela busca linear do Passo 6,

$$
\varphi_{\mu_{k}}\left(x^{k, j+1}\right) \leq \varphi_{\mu_{k}}\left(x^{k, j}\right)+\gamma \alpha_{k, j-1} \nabla \varphi_{\mu_{k}}\left(x^{k, j}\right)^{T} d_{x}^{k, j} .
$$


Não obstante, pelas Hipóteses 2 e 3 , as sequências $\left\{f\left(x^{k, j+1}\right)\right\},\left\{x_{i}^{k, j+1}-\ell_{i}\right\}$, para todo $i \in \mathcal{I}_{\ell}$ e $\left\{u_{i}-x_{i}^{k, j+1}\right\}$, para todo $i \in \mathcal{I}_{u}$, são limitadas. Deste modo, por (2.32) e pela definição da função $\varphi_{\mu}(\cdot) \operatorname{em~}(2.9)$

$$
\lim _{j \in \mathcal{J}} \varphi_{\mu_{k}}\left(x^{k, j+1}\right)=+\infty
$$

o que contradiz (2.33). Analogamente, vale o mesmo se existir um conjunto infinito $\mathcal{J} \subset \mathbb{N}$ e um índice $\hat{i} \in \mathcal{I}_{u}$ tais que $\left\{x_{\hat{i}}^{k, j+1}\right\}_{j \in \mathcal{J}} \rightarrow u_{\hat{i}}$.

Quanto à parte (II), basta notarmos que o Passo 8 implica que $z_{\ell}^{k, j+1}$ e $z_{u}^{k, j+1}$ satisfazem as relações (2.22) para todo $j$. Este fato e a parte (I) deste lema juntos implicam o que queríamos demonstrar.

Lema 10. A sequência $\left\{\bar{H}_{k, j}\right\}$ gerada pelo Algoritmo 1 é limitada.

Demonstração. Pelas Hipóteses 2 e 3, temos que a sequência $\left\{\nabla^{2} f\left(x^{k, j}\right)\right\}$ gerada pelo Algoritmo 1 é limitada. Mais ainda, a Hipótese 3 e o Lema $9(\mathrm{I})$ implicam que a sequência $\left\{L_{k, j}^{\dagger}, U_{k, j}^{\dagger}\right\}$ gerada pelo Algoritmo 1 é limitada. Não obstante, o Lema 9(II) implica que a sequência $\left\{Z_{k, j}^{L}, Z_{k, j}^{U}\right\}$ gerada pelo Algoritmo 1 é limitada. Finalmente, o Algoritmo 3 implica que $\xi_{k, j}=0$ ou $0 \neq \xi_{k, j} \in\left[\xi_{\min }, \xi_{\max }\right]$. Por conseguinte, segue o resultado.

Lema 11. Suponhamos que as Hipóteses 1, 2 e 3 valem. Então, a sequência $\left\{d_{x}^{k, j}, \lambda^{k, j+1}, z_{\ell}^{k, j+1}, z_{u}^{k, j+1}\right\}$ gerada pelo Algoritmo 1 é limitada.

Demonstração. Pelo Lema 9(II), temos que a sequência $\left\{z_{\ell}^{k, j+1}, z_{u}^{k, j+1}\right\}$ é limitada. Então, a fim de construir uma contradição, suponhamos que existe $\mathcal{J} \subset \mathbb{N}$ tal que

$$
\lim _{j \in \mathcal{J}}\left\|\left(d_{x}^{k, j}, \lambda^{k, j}+d_{\lambda}^{k, j}\right)\right\|=+\infty
$$

haja visto que, por (2.16b), $\lambda^{k, j+1}=\lambda^{k, j}+d_{\lambda}^{k, j}$. Primeiro, notemos que as Hipóteses 2 e 3 e o Lema 9(I) implicam que a sequência

$$
\left\{\nabla \varphi_{\mu_{k}}\left(x^{k, j}\right)\right\}_{j \in \mathcal{J}}=\left\{\nabla f\left(x^{k, j}\right)-\mu_{k} L_{k, j}^{\dagger} e+\mu_{k} U_{k, j}^{\dagger} e\right\}_{j \in \mathcal{J}}
$$

é limitada. Isto, junto com os Lemas 9 (II) e 10 acarreta a existência de $\hat{\mathcal{J}} \underset{\infty}{\subset \mathcal{J}}$ tal que

$$
\begin{aligned}
\lim _{j \in \mathcal{\mathcal { J }}} \nabla \varphi_{\mu_{k}}\left(x^{k, j}\right) & =\nabla \varphi_{\mu_{k}}\left(x^{k, *}\right), \\
\lim _{j \in \mathcal{\mathcal { J }}}\left(z_{\ell}^{k, j}, z_{u}^{k, j}\right) & =\left(z_{\ell}^{k, *}, z_{u}^{k, *}\right), \\
\lim _{j \in \hat{\mathcal{J}}} \bar{H}_{k, j} & =\bar{H}_{k, *} .
\end{aligned}
$$

Podemos reescrever o sistema (2.31) e obter

$$
M_{k, j}\left(\begin{array}{c}
d_{x}^{k, j} \\
\lambda^{k, j}+d_{\lambda}^{k, j}
\end{array}\right)=-\left(\begin{array}{c}
\nabla \varphi_{\mu_{k}}\left(x^{k, j}\right) \\
0
\end{array}\right),
$$

sendo que (2.36) implica que

$$
\lim _{j \in \hat{\mathcal{J}}} M_{k, j}=M_{k, *} .
$$


Por (2.35) temos que o lado direito do sistema (2.37) possui limite para $j \in \hat{\mathcal{J}}$. Além disso, o Passo 2 do Algoritmo 1 garante que $M_{k, j}$ será não singular para todo $j$. Por conseguinte, de (2.37) segue que

$$
\lim _{j \in \hat{\mathcal{J}}}\left(\begin{array}{c}
d^{k, j} \\
\lambda^{k, j}+d_{\lambda}^{k, j}
\end{array}\right)=\lim _{j \in \hat{\mathcal{J}}}-M_{k, j}^{-1}\left(\begin{array}{c}
\nabla \varphi_{\mu_{k}}\left(x^{k, j}\right) \\
0
\end{array}\right)=-M_{k, *}^{-1}\left(\begin{array}{c}
\nabla \varphi_{\mu_{k}}\left(x^{k, *}\right) \\
0
\end{array}\right),
$$

contradizendo (2.34).

Lema 12. Suponhamos que as Hipóteses 1, 2, 3 e 4 valem. Então, a sequência $\left\{d_{x}^{k, j}\right\}$ gerada pelo Algoritmo 1 tende a zero quando $j$ tende a infinito.

Demonstração. Pelo Lema 11, a sequência $\left\{d_{x}^{k, j}\right\}$ é limitada, portanto possui alguma subsequência convergente. Consideremos, por contradição, que existe um conjunto infinito $\mathcal{J} \subset \underset{\infty}{\subset \mathbb{N}}$ tal que

$$
\lim _{j \in \mathcal{J}} d_{x}^{k, j}=d_{x}^{k, *} \neq 0
$$

Pelo Lema 10, pela Hipótese 3 e pelo Lema 11, existe $\hat{\mathcal{J}} \underset{\infty}{\subset \mathcal{J}}$ tal que

$$
\lim _{j \in \hat{\mathcal{J}}} \bar{H}_{k, j}=\bar{H}_{k, *} \text { e } \lim _{j \in \hat{\mathcal{J}}}\left(x^{k, j}, \lambda^{k, j}, z_{\ell}^{k, j}, z_{u}^{k, j}\right)=\left(x^{k, *}, \lambda^{k, *}, z_{\ell}^{k, *}, z_{u}^{k, *}\right)
$$

Pré-multiplicando o primeiro bloco de equações do sistema (2.31) por $d_{x}^{k, j}$, que, pelo segundo bloco de equações do sistema (2.31), pertence ao núcleo da matriz $A$, vem que

$$
\begin{aligned}
\left(d_{x}^{k, j}\right)^{T} \bar{H}_{k, j} d_{x}^{k, j} & =-\left(d_{x}^{k, j}\right)^{T} \nabla \varphi_{\mu_{k}}\left(x^{k, j}\right) \\
& \geq \sigma\left\|d_{x}^{k, j}\right\|^{2} \text {, pela Hipótese } 4 .
\end{aligned}
$$

Logo, temos que

$$
\nabla \varphi_{\mu_{k}}\left(x^{k, j}\right)^{T} d_{x}^{k, j} \leq-\sigma\left\|d_{x}^{k, j}\right\|^{2}
$$

Passando ao limite em (2.39) para $j \in \hat{\mathcal{J}}$, vem que

$$
\nabla \varphi_{\mu_{k}}\left(x^{k, *}\right)^{T} d_{x}^{k, *} \leq-\sigma\left\|d_{x}^{k, *}\right\|^{2} .
$$

Pelo Lema 9(I), temos que $\ell_{i}+\delta \leq x_{i}^{k, j}$, para todo $i \in \mathcal{I}_{\ell}$ e $x_{i}^{k, j} \leq u_{i}-\delta$, para todo $i \in \mathcal{I}_{u}$, com $\delta>0$. Tomando o limite para $j \in \hat{\mathcal{J}}$, temos que $\ell_{i}<x_{i}^{k, *}$, para todo $i \in \mathcal{I}_{\ell}$ e $x_{i}^{k, *}<u_{i}$, para todo $i \in \mathcal{I}_{u}$. Portanto, existe $\hat{\alpha} \in(0,1]$ tal que, para todo $\alpha \in(0, \hat{\alpha}]$,

$$
\ell_{i}<x_{i}^{k, *}+\alpha\left[d_{x}^{k, *}\right]_{i} \text {, para todo } i \in \mathcal{I}_{\ell} \text { e } x_{i}^{k, *}+\alpha\left[d_{x}^{k, *}\right]_{i}<u_{i} \text {, para todo } i \in \mathcal{I}_{u} .
$$

Como $d_{x}^{k, *} \neq 0$ e (2.40) implica que $\nabla \varphi_{\mu_{k}}\left(x^{k, *}\right)^{T} d_{x}^{k, *}<0$, então existe $\tilde{\alpha} \in(0, \hat{\alpha}]$ tal que para todo $\alpha \in(0, \tilde{\alpha}]$ vale $(2.41) \mathrm{e}$

$$
\varphi_{\mu_{k}}\left(x^{k, *}+\alpha d_{x}^{k, *}\right) \leq \varphi_{\mu_{k}}\left(x^{k, *}\right)+\bar{\gamma} \alpha \nabla \varphi_{\mu_{k}}\left(x^{k, *}\right)^{T} d_{x}^{k, *},
$$

com $\bar{\gamma} \in(\gamma, 1)$. Notemos que esta é uma condição de decréscimo suficiente, o que assegura a existência de $\tilde{\alpha}$ (veja Lema 7), entretanto é uma condição mais rigorosa que a exigida no Passo 6 do Algoritmo 1, já que $\bar{\gamma}>\gamma$. Como $\varphi_{\mu}(\cdot)$ é uma função continuamente diferenciável, da interioridade estrita (2.41) e do fato de $d_{x}^{k, *}$ ser uma direção de descida a partir de $x^{k, *}$, conforme (2.40), definimos

$$
\rho^{*}=\min \{\rho \in\{0,1,2, \ldots\}\}
$$


tal que

$$
\alpha_{k, *} \stackrel{\text { def }}{=} \tilde{\alpha}\left(\frac{1}{2}\right)^{\rho^{*}} \leq \alpha_{k, j}
$$

$\mathrm{e}$

$$
\varphi_{\mu_{k}}\left(x^{k, j}+\alpha_{k, *} d_{x}^{k, j}\right) \leq \varphi_{\mu_{k}}\left(x^{k, j}\right)+\gamma \alpha_{k, *} \nabla \varphi_{\mu_{k}}\left(x^{k, j}\right)^{T} d_{x}^{k, j}
$$

para todo $j \in \hat{\mathcal{J}}, j$ suficientemente grande. Logo

$$
\begin{aligned}
\varphi_{\mu_{k}}\left(x^{k, j+1}\right) & \leq \varphi_{\mu_{k}}\left(x^{k, j}\right)+\gamma \alpha_{k, j} \nabla \varphi_{\mu_{k}}\left(x^{k, j}\right)^{T} d_{x}^{k, j} \\
& \leq \varphi_{\mu_{k}}\left(x^{k, j}\right)-\gamma \alpha_{k, j} \sigma\left\|d_{x}^{k, j}\right\|^{2}, \text { por }(2.39) \\
& \leq \varphi_{\mu_{k}}\left(x^{k, j}\right)-\frac{1}{2} \gamma \alpha_{k, j} \sigma\left\|d_{x}^{k, *}\right\|^{2}, \text { por }(2.38) \\
& \leq \varphi_{\mu_{k}}\left(x^{k, j}\right)-\frac{1}{2} \gamma \alpha_{k, *} \sigma\left\|d_{x}^{k, *}\right\|^{2} \text {, para } j \text { suficientemente grande, }
\end{aligned}
$$

o que implica que

$$
\lim _{j \in \hat{\mathcal{J}}} \varphi_{\mu_{k}}\left(x^{k, j}\right)=-\infty
$$

contradizendo as Hipóteses 2 e 3.

O teorema a seguir estabelece o resultado de convergência global do Algoritmo 1.

Teorema 13. Suponhamos que as Hipóteses 1, 2, 3 e 4 valem. Então, qualquer ponto limite da sequência $\left\{x^{k, j}+\alpha_{k, j} d_{x}^{k, j}, \lambda^{k, j}+d_{\lambda}^{k, j}, z_{\ell}^{k, j}+\alpha_{k, j}^{z_{\ell}} d_{z_{\ell}}^{k, j}, z_{u}^{k, j}+\alpha_{k, j}^{z_{u}} d_{z_{u}}^{k, j}\right\}$ gerada pelo Algoritmo 1 satisfaz as condiçôes de otimalidade de primeira ordem (2.8) do subproblema (2.10).

Demonstração. Seja $\left(x^{k, *}, \lambda^{k, *}, z_{\ell}^{k, *}, z_{u}^{k, *}\right)$ um ponto limite qualquer da sequência

$$
\left\{x^{k, j}+\alpha_{k, j} d_{x}^{k, j}, \lambda^{k, j}+d_{\lambda}^{k, j}, z_{\ell}^{k, j}+\alpha_{k, j}^{z_{\ell}} d_{z_{\ell}}^{k, j}, z_{u}^{k, j}+\alpha_{k, j}^{z_{u}} d_{z_{u}}^{k, j}\right\},
$$

digamos para a subsequência cujos índices pertencem ao conjunto $\mathcal{J} \subset \mathbb{N}$. Passando ao limite em (2.31) para $j \in \mathcal{J}$, pelo Lema 12 vem que

$$
A^{T} \lambda^{k, *}=-\nabla \varphi_{\mu_{k}}\left(x^{k, *}\right)=-\nabla f\left(x^{k, *}\right)+\mu_{k} L_{k, *}^{\dagger} e-\mu_{k} U_{k, *}^{\dagger} e,
$$

ou seja,

$$
\nabla f\left(x^{k, *}\right)+A^{T} \lambda^{k, *}-\mu_{k} L_{k, *}^{\dagger} e+\mu_{k} U_{k, *}^{\dagger} e=0 .
$$

O Lema 12 implica que, para $j \in \mathcal{J}$ suficientemente grande, $d_{x}^{k, j}$ será suficientemente pequena, e, por isso, as condições (3.17) serão satisfeitas. Por conseguinte, $\alpha_{k, j}^{z_{\ell}}=\alpha_{k, j}^{z_{u}}=1 \mathrm{e}$, passando ao limite em (2.13) para $j \in \mathcal{J}$, por (2.16) temos que

$$
z_{\ell}^{k, *}=\mu_{k} L_{k, *}^{\dagger} e \text { e } z_{u}^{k, *}=\mu_{k} U_{k, *}^{\dagger} e .
$$

Deste modo, (2.43) e (2.44) juntos implicam que

$$
\begin{aligned}
\nabla f\left(x^{k, *}\right)+A^{T} \lambda^{k, *}-z_{\ell}^{k, *}+z_{u}^{k, *} & =0 \\
L_{k, *} z_{l, *}^{k, \mu_{k}} e & =0 \\
U_{k, *} z_{u}^{k, *}-\mu_{k} e & =0 .
\end{aligned}
$$

Logo, como $A x^{k, *}=b$, por (2.45) temos que (2.8) se cumpre em $\left(x^{k, *}, \lambda^{k, *}, z_{\ell}^{k, *}, z_{u}^{k, *}\right)$. 


\subsection{Modelo algorítmico para a resolução do problema}

Nesta seção, apresentamos o método para a resolução do problema (2.1).

Algoritmo 2. Método de pontos interiores - Algoritmo externo.

Entrada: $x^{0} \in \mathcal{F}_{0}, \lambda^{0} \in \mathbb{R}^{m}, z_{\ell}^{0} \in \mathbb{R}^{n}$ e $z_{u}^{0} \in \mathbb{R}^{n}$ tais que $\left[z_{\ell}^{0}\right]_{i}>0$ para $i \in \mathcal{I}_{\ell}$ e $\left[z_{u}^{0}\right]_{i}>0$ para $i \in \mathcal{I}_{u}$ e $\mu_{0}>0$; constantes $\varepsilon_{\text {tol }}>0, \kappa_{\varepsilon}>0, \kappa_{\mu} \in(0,1), \theta_{\mu} \in(1,2) ; k \leftarrow 0$.

Passo 1. Encontre uma solução aproximada $x^{k+1}$ de

$$
\begin{array}{cl}
\min _{x} & \varphi_{\mu_{k}}(x) \\
\text { s.a } & A x=b
\end{array}
$$

e $\lambda^{k+1}, z_{\ell}^{k+1}$ e $z_{u}^{k+1}$ tais que $E_{\mu_{k}}\left(x^{k+1}, \lambda^{k+1}, z_{\ell}^{k+1}, z_{u}^{k+1}\right) \leq \kappa_{\varepsilon} \mu_{k}$.

Passo 2. Compute $\mu_{k+1}=\min \left\{\kappa_{\mu} \mu_{k}, \mu_{k}^{\theta_{\mu}}\right\}$.

Passo 3. Faça $k \leftarrow k+1$ e volte ao Passo 1 .

\subsubsection{Boa definição}

Em primeiro lugar, a Hipótese 1 garante que é possível encontrar $x^{0} \in \mathcal{F}_{0}$. Deste modo, a boa definição do Algoritmo 2 é condicionada pela execução, bem definida e em tempo finito, do método a ser usado no Passo 1, que é o Algoritmo 1. Como a Proposição 8 estabelece a boa definição do Algoritmo 1, nos resta apresentar o seguinte lema.

Lema 14. Sejam válidas as Hipóteses 1, 2, 3 e 4. Então, para todo $k$, é possível encontrar $\left(x^{k+1}, \lambda^{k+1}, z_{\ell}^{k+1}, z_{u}^{k+1}\right)$, tal que

$$
E_{\mu_{k}}\left(x^{k+1}, \lambda^{k+1}, z_{\ell}^{k+1}, z_{u}^{k+1}\right) \leq \kappa_{\varepsilon} \mu_{k},
$$

em uma quantidade finita de passos do Algoritmo 1.

Demonstração. Primeiro, notemos que $\mu_{0}>0$ e, pelo Passo $2, \mu_{k}>0$ para todo $k$. Portanto, temos que $\kappa_{\varepsilon} \mu_{k}>0$ para todo $k$. Não obstante, para cada $k$, o Teorema 13 implica que, com $j$ tendendo ao infinito, o Algoritmo 1 é capaz de encontrar $\left(x^{k, *}, \lambda^{k, *}, z_{\ell}^{k, *}, z_{u}^{k, *}\right)$ tal que $E_{\mu_{k}}\left(x^{k, *}, \lambda^{k, *}, z_{\ell}^{k, *}, z_{u}^{k, *}\right)=0$ (de acordo com (2.45)). Por conseguinte, para $j$ suficientemente grande, o Algoritmo 1 é capaz de encontrar um ponto $\left(x^{k, j}, \lambda^{k, j}, z_{\ell}^{k, j}, z_{u}^{k, j}\right)$ tal que $E_{\mu_{k}}\left(x^{k, j}, \lambda^{k, j}, z_{\ell}^{k, j}, z_{u}^{k, j}\right) \leq \kappa_{\varepsilon} \mu_{k}$. Deste modo, é possível obter $\left(x^{k+1}, \lambda^{k+1}, z_{\ell}^{k+1}, z_{u}^{k+1}\right)=\left(x^{k, j}, \lambda^{k, j}, z_{\ell}^{k, j}, z_{u}^{k, j}\right)$ em uma quantidade finita de passos do Algoritmo 1.

Assim sendo, o Lema 14 implica a boa definição do Algoritmo 2, que é estabelecida a seguir.

Proposição 15. Se as Hipóteses 1, 2, 3 e 4 valem, então o Algoritmo 2 está bem definido. 


\subsubsection{Análise da convergência global}

O seguinte resultado nos dá a convergência global do Algoritmo 2.

Teorema 16. Suponhamos que o Algoritmo 2 gera uma sequência infinita de iterandos e que as Hipóteses 1, 2, 3 e 4 valem para todo $\mu_{k}$ com as mesmas constantes para os limitantes. Se a sequência gerada pelo Algoritmo 2 admitir algum ponto limite $\left(x^{*}, \lambda^{*}, z_{\ell}^{*}, z_{u}^{*}\right)$, então

$$
E_{0}\left(x^{*}, \lambda^{*}, z_{\ell}^{*}, z_{u}^{*}\right)=0
$$

com $E_{\mu}\left(x, \lambda, z_{\ell}, z_{u}\right)$ como definido em (2.24).

Demonstração. Seja $\mathcal{K} \underset{\infty}{\subset} \mathbb{N}$ um conjunto infinito tal que

$$
\lim _{k \in \mathcal{K}}\left(x^{k+1}, \lambda^{k+1}, z_{\ell}^{k+1}, z_{u}^{k+1}\right)=\left(x^{*}, \lambda^{*}, z_{\ell}^{*}, z_{u}^{*}\right) .
$$

Suponhamos que (2.46) não vale. Deste modo

$$
\begin{aligned}
\left\|\nabla f\left(x^{*}\right)+A^{T} \lambda^{*}-z_{\ell}^{*}+z_{u}^{*}\right\|_{\infty} & >0 \text { ou } \\
\left\|L_{*} Z_{*}^{\ell} e\right\|_{\infty} & >0 \text { ou } \\
\left\|U_{*} Z_{*}^{u} e\right\|_{\infty} & >0 .
\end{aligned}
$$

Pelo Passo 1 do Algoritmo 2, temos que

$$
E_{\mu_{k}}\left(x^{k+1}, \lambda^{k+1}, z_{\ell}^{k+1}, z_{u}^{k+1}\right) \leq \kappa_{\varepsilon} \mu_{k},
$$

para todo $k$, isto é,

$$
\begin{aligned}
\left\|\nabla f\left(x^{k+1}\right)+A^{T} \lambda^{k+1}-z_{\ell}^{k+1}+z_{u}^{k+1}\right\|_{\infty} & \leq \kappa_{\varepsilon} \mu_{k} \mathrm{e} \\
\left\|L_{k+1} Z_{k+1}^{\ell} e-\mu_{k} e\right\|_{\infty} & \leq \kappa_{\varepsilon} \mu_{k} \mathrm{e} \\
\left\|U_{k+1} Z_{k+1}^{u} e-\mu_{k} e\right\|_{\infty} & \leq \kappa_{\varepsilon} \mu_{k},
\end{aligned}
$$

para todo $k$. Como $\mathcal{K} \subset \mathbb{\infty}$, temos, pelo Passo 2 do Algoritmo 2, que

$$
\lim _{k \in \mathcal{K}} \mu_{k}=0
$$

De (2.47), (2.48b) e (2.49b), para todo $k$ suficientemente grande temos que

$$
0<\left\|L_{k+1} Z_{k+1}^{\ell} e\right\|_{\infty}-\mu_{k} \leq \kappa_{\varepsilon} \mu_{k},
$$

ou seja,

$$
0<\mu_{k}\left(\kappa_{\varepsilon}+1\right)
$$

Deste modo, chegamos a um absurdo, já que (2.50) vale. De maneira análoga, de (2.47), (2.48c) e (2.49c) chegamos à mesma contradição. Não obstante, de (2.47), (2.48a) e (2.49a), para todo $k$ suficientemente grande

$$
0<\left\|\nabla f\left(x^{k+1}\right)+A^{T} \lambda^{k+1}-z_{\ell}^{k+1}+z_{u}^{k+1}\right\|_{\infty} \leq \kappa_{\varepsilon} \mu_{k},
$$

isto é,

$$
0<\kappa_{\varepsilon} \mu_{k}
$$

o que nos dá uma contradição similar. Por conseguinte, (2.48) não ocorre. Logo, vale (2.46). 
Notemos que o resultado obtido no Teorema 16 implica que, dada qualquer sequência gerada pelo Algoritmo 2, se essa sequência admitir ponto limite, então este ponto limite satisfaz (2.8) para $\mu_{k}=0$. Deste modo, temos que este ponto limite satisfaz (2.3), já que $z_{\ell}^{*}$ e $z_{u}^{*}$ são não negativos, pela definição do método, e satisfazem as relações de complementaridade por (2.8c) e (2.8d) com $\mu_{k}=0$. Por conseguinte, temos o seguinte corolário.

Corolário 17. Suponhamos que o Algoritmo 2 gera uma sequência infinita de iterandos e que as Hipóteses 1, 2, 3 e 4 valem para todo $\mu_{k}$ com as mesmas constantes para os limitantes. Se a sequência gerada pelo Algoritmo 2 admitir algum ponto limite $\left(x^{*}, \lambda^{*}, z_{\ell}^{*}, z_{u}^{*}\right)$, então este ponto é um ponto KKT para o problema (2.1). 


\section{Capítulo 3}

\section{Detalhes de implementação}

O algoritmo apresentado neste trabalho foi implementado usando Fortran 90 [14, 20], e a esta implementação demos o nome de LCMIN. Neste capítulo, apresentaremos alguns detalhes desta implementação.

\subsection{Critérios de parada}

No método apresentado no Algoritmo 2, o objetivo é parar quando um ponto aproximadamente estacionário para o problema (2.1) for encontrado, isto é, quando um ponto satisfizer aproximadamente as condições KKT (2.3) do problema (2.1). Por outro lado, para cada subproblema, paramos quando o resíduo do sistema (2.8) for suficientemente pequeno (veja Passo 1 do Algoritmo 2). A fim de facilitar esta parada e possibilitar aquela, definimos

$$
E_{\mu}^{s}\left(x, \lambda, z_{\ell}, z_{u}\right)=\max \left\{\frac{\left\|\nabla f(x)+A^{T} \lambda-z_{\ell}+z_{u}\right\|_{\infty}}{s_{d}}, \frac{\left\|L Z^{\ell} e-\mu e\right\|_{\infty}}{s_{\ell}}, \frac{\left\|U Z^{u} e-\mu e\right\|_{\infty}}{s_{u}}\right\}
$$

como sendo o erro escalado do sistema (2.8) (sem considerar a viabilidade (2.8b), já que todo $x^{k}$ satisfaz $A x^{k}=b$ ), onde

$$
s_{d}=\max \left\{s_{\max }, \frac{\|\lambda\|_{1}+\left\|z_{\ell}\right\|_{1}+\left\|z_{u}\right\|_{1}}{m+2 n}\right\} / s_{\max }
$$

$\mathrm{e}$

$$
s_{\ell}=\max \left\{s_{\max }, \frac{\left\|z_{\ell}\right\|_{1}}{n}\right\} / s_{\max } \quad \text { e } \quad s_{u}=\max \left\{s_{\max }, \frac{\left\|z_{u}\right\|_{1}}{n}\right\} / s_{\max }
$$

de forma que $s_{\max } \geq 1$ é uma constante definida (utilizaremos $s_{\max }=100$ ), e consideraremos que um ponto $\left(x^{k}, \lambda^{k}, z_{\ell}^{k}, z_{u}^{k}\right)$ é aproximadamente estacionário para o problema (2.1) quando

$$
E_{0}^{s}\left(x^{k}, \lambda^{k}, z_{\ell}^{k}, z_{u}^{k}\right) \leq \varepsilon_{\mathrm{tol}}
$$

com $\varepsilon_{\text {tol }}>0$ (na prática, consideramos $\varepsilon_{\text {tol }}=10^{-8}$ ). Tal critério de parada implica que as equações $(2.3 \mathrm{a}, 2.3 \mathrm{~g}, 2.3 \mathrm{~h})$ são aproximadamente satisfeitas (com $\nu_{\ell}=z_{\ell}$ e $\nu_{u}=z_{u}$ ), enquanto as equações $(2.3 \mathrm{~b}, 2.3 \mathrm{c}, 2.3 \mathrm{~d}, 2.3 \mathrm{e}, 2.3 \mathrm{f})$ são satisfeitas pelo ponto inicial e pelos pontos gerados pelo método, já que, para todo $k, x^{k}$ satisfaz $A x^{k}=b$ e $\left[z_{\ell}^{k}\right]_{i} \geq 0$, para $i \in \mathcal{I}_{\ell}$ e $\left[z_{u}^{k}\right]_{i} \geq 0$, para $i \in \mathcal{I}_{u}$. Logo, as condições de otimalidade (2.3) do problema (2.1) são aproximadamente satisfeitas em $\left(x^{k}, \lambda^{k}, z_{\ell}^{k}, z_{u}^{k}\right)$. Os fatores $s_{d}, s_{\ell}$ e $s_{u}$ calculados fazem com que o erro de otimalidade (3.1) seja controlado quando os multiplicadores $\lambda, z_{\ell}$ e $z_{u}$ tomarem valores arbitrariamente grandes. 
De maneira análoga, para cada subproblema (2.4), consideraremos que $\left(x^{k, j}, \lambda^{k, j}, z_{\ell}^{k, j}, z_{u}^{k, j}\right)$ é um ponto aproximadamente estacionário quando

$$
E_{\mu_{k}}^{s}\left(x^{k, j}, \lambda^{k, j}, z_{\ell}^{k, j}, z_{u}^{k, j}\right) \leq \kappa_{\varepsilon} \mu_{k},
$$

com $\kappa_{\varepsilon}>0$ (na prática, consideramos $\kappa_{\varepsilon}=10$ ). Assim sendo, este será o critério considerado no Passo 1 do Algoritmo 2. Notemos que

$$
E_{\mu}^{s}\left(x, \lambda, z_{\ell}, z_{u}\right) \leq E_{\mu}\left(x, \lambda, z_{\ell}, z_{u}\right),
$$

com $E_{\mu}$ definido em (2.24). Portanto, ao considerar o critério de parada (3.4) ao invés de (2.23), estamos potencialmente adiantando a parada no Passo 1 do Algoritmo 2.

A fim de evitar problemas numéricos, a cada subproblema o novo parâmetro barreira é obtido como

$$
\mu_{k+1}=\max \left\{\frac{\varepsilon_{\mathrm{tol}}}{10}, \min \left\{\kappa_{\mu} \mu_{k}, \mu_{k}^{\theta_{\mu}}\right\}\right\}
$$

onde $\kappa_{\mu} \in(0,1)$ e $\theta_{\mu} \in(1,2)$ como em $(2.25)$ (na prática, $\kappa_{\mu}=0.2$ e $\theta_{\mu}=1.5$ ). Esta atualização faz com que $\mu_{k}$ não assuma valores demasiadamente pequenos, desnecessários para satisfazer (3.3), evitando problemas numéricos em subproblemas no final do processo de otimização.

Além dos critérios referentes à otimalidade, usamos alguns critérios de salvaguarda. Primeiro, declaramos parada quando, no Algoritmo 1,

$$
\left\|x^{k, j}\right\|_{\infty} \geq \kappa_{x}
$$

onde $\kappa_{x}$ é um valor muito grande (na prática, consideramos $\kappa_{x}=10^{20}$ ). A satisfação deste critério significa que os iterandos estão divergindo, o que indica que a função objetivo do problema pode ser ilimitada.

Por outro lado, paramos sempre que uma quantidade máxima de iterações for excedida. Neste contexto, o processo de otimização é encerrado se

$$
k \geq k_{\max }
$$

para algum $k_{\max }>0$ (na prática, consideramos $k_{\max }=50$ ), isto é, caso o Algoritmo 2 exceder um limite de iterações, ou se

$$
j \geq j_{\max } \text { e } \mu_{k}=\frac{\varepsilon_{\text {tol }}}{10}
$$

para algum $j_{\max }>0$ (na prática, tomamos $j_{\max }=200$ ), ou seja, caso o Algoritmo 1 exceder uma quantidade máxima de iterações e o parâmetro barreira for o menor possível, de acordo com a regra de atualização do mesmo (3.5). Caso a quantidade máxima de iterações do Algoritmo 1 for excedida e o parâmetro barreira não for o menor possível, ele é atualizado de acordo com (3.5) e a resolução de um novo subproblema se inicia.

\subsection{Escalamento do problema original}

Como a direção de Newton computada em (2.14) é invariante ao escalamento da função objetivo e das restrições, substituiremos $f, A$ e $b$ no problema (2.1) por $s_{f} f(x), S_{A} A$ e $S_{A} b$, respectivamente, $\operatorname{com} s_{f}>0$ e $S_{A}=\operatorname{diag}\left(s_{1}^{A}, \ldots, s_{m}^{A}\right)$.

A estratégia de escalamento que adotamos aqui pressupõe que o problema já possui um bom escalamento e não exigirá modificações, a não ser em casos extremos. Assim sendo, partindo do limiar $g_{\max }>0$ (utilizaremos $\left.g_{\max }=100\right)$, definimos

$$
s_{f}=\min \left\{1, \frac{g_{\max }}{\left\|\nabla f\left(x^{0}\right)\right\|_{\infty}}\right\}
$$


onde $x^{0}$ é o ponto inicial computado usando o método da Seção 3.3, e

$$
s_{i}^{A}=\min \left\{1, \frac{g_{\max }}{\left\|a_{i}^{T}\right\|_{\infty}}\right\}, \quad \text { para } i=1, \ldots, m
$$

onde $a_{i}^{T}$ é a $i$-ésima linha da matriz $A$.

Suponhamos que LCMIN encontre um ponto aproximadamente estacionário $\left(x^{*}, \lambda^{*}, z_{\ell}^{*}, z_{u}^{*}\right)$ para o problema (2.1) escalado. Naturalmente, devemos obter $\left(\bar{x}^{*}, \bar{\lambda}^{*}, \bar{z}_{\ell}^{*}, \bar{z}_{u}^{*}\right)$ de $\left(x^{*}, \lambda^{*}, z_{\ell}^{*}, z_{u}^{*}\right)$ que seja uma solução para o problema (2.1) original, não escalado. Temos que

$$
E_{0}^{s}\left(x^{*}, \lambda^{*}, z_{\ell}^{*}, z_{u}^{*}\right) \leq \varepsilon_{\text {tol }}
$$

com os respectivos valores escalados em (3.1). Assim sendo, temos que

$$
\left\|s_{f} \nabla f\left(x^{*}\right)+S_{A} A^{T} \lambda^{*}-z_{\ell}^{*}+z_{u}^{*}\right\|_{\infty} \leq \varepsilon_{\mathrm{tol}},
$$

ou seja,

$$
s_{f}\left\|\nabla f\left(x^{*}\right)+A^{T}\left(\frac{1}{s_{f}} S_{A} \lambda^{*}\right)-\left(\frac{1}{s_{f}} z_{\ell}^{*}\right)+\left(\frac{1}{s_{f}} z_{u}^{*}\right)\right\| \leq \varepsilon_{\mathrm{tol}} .
$$

Por conseguinte, se tomarmos

$$
\bar{x}^{*}=x^{*}, \bar{\lambda}^{*}=\frac{1}{s_{f}} S_{A} \lambda^{*}, \bar{z}_{\ell}^{*}=\frac{1}{s_{f}} z_{\ell}^{*} \text { e } \bar{z}_{u}^{*}=\frac{1}{s_{f}} z_{u}^{*}
$$

vem que

$$
\left\|\nabla f\left(\bar{x}^{*}\right)+A^{T} \bar{\lambda}^{*}-\bar{z}_{\ell}{ }^{*}+{\overline{z_{u}}}^{*}\right\|_{\infty}=\frac{1}{s_{f}}\left\|s_{f} \nabla f\left(x^{*}\right)+S_{A} A^{T} \lambda^{*}-z_{\ell}^{*}+z_{u}^{*}\right\|_{\infty} \leq \frac{1}{s_{f}} \varepsilon_{\mathrm{tol}} .
$$

Assim sendo, definindo $\left(\bar{x}^{*}, \bar{\lambda}^{*}, \bar{z}_{\ell}^{*}, \bar{z}_{u}^{*}\right)$ de acordo com (3.7), temos que

$$
E_{0}^{s}\left(\bar{x}^{*}, \bar{\lambda}^{*}, \bar{z}_{\ell}^{*}, \bar{z}_{u}^{*}\right) \leq \frac{1}{s_{f}} \varepsilon_{\mathrm{tol}},
$$

isto é, o ponto satisfaz uma tolerância de otimalidade, não escalada, para o problema original.

Logo, é importante notar que, ao optar por resolver um problema escalado, com uma constante $s_{f}$ para escalamento da função objetivo, a solução encontrada satisfará a precisão de otimalidade em $\varepsilon_{\text {tol }} / s_{f}$, ou seja, será menos precisa quando $s_{f}<1$. Na prática, observa-se que um algoritmo de otimização, suas estratégias de inicialização e de parada costumam comportar-se de maneira distinta frente a diferentes fatores de escala do problema original, de maneira especial se tais fatores forem extremos, isto é, muito pequenos ou muito grandes. Deste modo, pelo escalamento da função objetivo e das restrições, queremos que o erro de otimalidade que determina quando um determinado ponto é solução do problema, bem como o valor escolhido para a tolerância $\varepsilon_{\text {tol }}$, façam mais sentido numericamente.

\subsection{Computação do ponto inicial}

É comum que métodos computacionais de otimização iniciem sua execução com uma estimativa inicial da solução $\bar{x}^{0}$ fornecida pelo usuário. Todavia, métodos de pontos interiores pressupõem iterandos estritamente viáveis, em nosso caso, iterandos que pertençam ao conjunto $\mathcal{F}_{0} \neq \emptyset$. Neste contexto, não é natural pedir que o próprio usuário forneça um ponto inicial estritamente viável, mas o mais razoável seria calcular um ponto inicial que leve em conta, de alguma forma, a estimativa $\bar{x}^{0}$ dada pelo usuário. Nesta seção, nosso objetivo é exibir meios para chegar neste objetivo. 


\subsubsection{Computação do ponto inicial usando programação linear}

Esta estratégia consiste em encontrar $x^{0}$ como solução do seguinte problema:

$$
\begin{array}{cl}
\min _{x^{0}} & \left\|x^{0}-\bar{x}^{0}\right\|_{1} \\
\text { s. a } & A x^{0}=b \\
& \ell_{i}+\left[\delta_{\ell}\right]_{i} \leq x_{i}^{0} \leq u_{i}-\left[\delta_{u}\right]_{i}, \text { para } i=1, \ldots, n,
\end{array}
$$

com $\delta_{\ell}, \delta_{u} \in \mathbb{R}_{+}^{n}$ perturbações para as restrições de caixa. A solução deste problema é um ponto $x^{0}$ tal que $A x^{0}=b, \ell<x^{0}<u$ e que está o mais próximo possível (na norma 1 ) do ponto $\bar{x}^{0}$ dado pelo usuário. Se considerarmos $r=x^{0}-\bar{x}^{0}$, podemos reescrever (3.8) como

$$
\begin{array}{ll}
\min _{r} & \|r\|_{1} \\
\text { s. a } & A r=b-A \bar{x}^{0} \\
& \ell_{i}+\left[\delta_{\ell}\right]_{i}-\bar{x}_{i}^{0} \leq r_{i} \leq u_{i}-\left[\delta_{u}\right]_{i}-\bar{x}_{i}^{0}, \text { para } i=1, \ldots, n .
\end{array}
$$

Fazendo a mudança de variáveis $r=v-w$, com $v_{i} \geq 0$ e $w_{i} \geq 0$ para todo $i=1, \ldots, n$, é possível reformular (3.9) como um problema de programação linear

$$
\begin{array}{rlrl}
\min _{v, w} \sum_{i=1}^{n} v_{i}+\sum_{i=1}^{n} w_{i} & & \\
\text { s. a } & A(v-w) & =b-A \bar{x}^{0} & \\
\ell_{i}+\left[\delta_{\ell}\right]_{i}-\bar{x}_{i}^{0} \leq v_{i}-w_{i} & \leq u_{i}-\left[\delta_{u}\right]_{i}-\bar{x}_{i}^{0}, & & \text { para } i=1, \ldots, n \\
v_{i} & \geq 0, & & \text { para } i=1, \ldots, n \\
w_{i} & \geq 0, & & \text { para } i=1, \ldots, n .
\end{array}
$$

Para eliminar do problema restrições do tipo $x_{i} \geq-\infty$ e $x_{i} \leq \infty$, consideraremos os conjuntos $\mathcal{I}_{\ell}$ e $\mathcal{I}_{u}$ definidos em $(2.2)$ e reescrevemos (3.10) como

$$
\begin{array}{rlrl}
\min _{v, w} \sum_{i=1}^{n} v_{i}+\sum_{i=1}^{n} w_{i} & & \\
\text { s. a } & A(v-w) & =b-A \bar{x}^{0} & \\
v_{i}-w_{i} & \geq \ell_{i}+\left[\delta_{\ell}\right]_{i}-\bar{x}_{i}^{0}, & & \text { para } i \in \mathcal{I}_{\ell} \\
-v_{i}+w_{i} & \geq-u_{i}+\left[\delta_{u}\right]_{i}+\bar{x}_{i}^{0}, & & \text { para } i \in \mathcal{I}_{u} \\
v_{i} & \geq 0, & & \text { para } i=1, \ldots, n \\
w_{i} & \geq 0, & & \text { para } i=1, \ldots, n .
\end{array}
$$

Encontrar uma solução $\left(v^{*}, w^{*}\right)$ para (3.11) equivale encontrar uma solução para (3.8). Desta forma, o ponto inicial desejado define-se como $x^{0}=\bar{x}^{0}+\left(v^{*}-w^{*}\right)$. Assim formulado, o problema (3.8) aproveita, de certa forma, o ponto inicial fornecido pelo usuário. As perturbações $\delta_{\ell}$ e $\delta_{u}$ garantem que $x^{0}$ satisfaça estritamente as restrições de caixa. Para calcular essas perturbações, seguiremos as sugestões em [25, p. 44], definindo

$$
\begin{aligned}
{\left[\delta_{\ell}\right]_{i} } & =\min \left\{\kappa_{1} \max \left\{1,\left|\ell_{i}\right|\right\}, \kappa_{2}\left(u_{i}-\ell_{i}\right)\right\} \\
{\left[\delta_{u}\right]_{i} } & =\min \left\{\kappa_{1} \max \left\{1,\left|u_{i}\right|\right\}, \kappa_{2}\left(u_{i}-\ell_{i}\right)\right\},
\end{aligned}
$$

para $i=1, \ldots, n$, com $\kappa_{1}>0$ e $\kappa_{2} \in\left(0, \frac{1}{2}\right)$. Na implementação, tomaremos $\kappa_{1}=\kappa_{2}=10^{-2}$. Assim calculada, temos a garantia que a perturbação aplicada à restrição de caixa inferior não fará com que esta se aproxime demais da superior, e vice-versa.

\subsubsection{Computação do ponto inicial resolvendo problemas de viabilidade}

Neste caso, computamos o ponto inicial $x^{0}$ como solução do problema de

$$
\begin{array}{ll}
\min _{x} & \|A x-b\|_{2}^{2} \\
\text { s. a } & \ell_{i}+\left[\delta_{\ell}\right]_{i} \leq x_{i} \leq u_{i}-\left[\delta_{u}\right]_{i}, \text { para } i=1, \ldots, n,
\end{array}
$$


com $\delta_{\ell}$ e $\delta_{u}$ como definidos em (3.12). Para resolver o problema (3.13), usamos o Algencan [1, 2] com a opção IGNore-objective-function, que faz uso da rotina GencAn [3, 8, 9]. Neste caso, fornecemos como ponto inicial o mesmo dado pelo usuário. Desta forma, o ponto inicial do usuário é aproveitado de certa forma na computação do ponto inicial para o nosso método.

\subsection{Estimativa inicial para os multiplicadores de Lagrange}

Tendo calculado o ponto inicial conforme a Seção 3.3 e atribuído valores iniciais para $z_{\ell}$ e $z_{u}$, é possível estimar os multiplicadores de Lagrange $\lambda$ das restrições de igualdade resolvendose o sistema linear indeterminado (2.8a) usando quadrados mínimos. Para isto, consideremos $c=\nabla f\left(x^{0}\right)-z_{\ell}+z_{u}$, queremos resolver o problema

$$
\min _{\lambda \in \mathbb{R}^{m}} q(\lambda)=\frac{1}{2}\left\|A^{T} \lambda+c\right\|^{2},
$$

cuja solução $\lambda^{*}$ será nossa estimativa inicial para os multiplicadores de Lagrange. O problema (3.14) é convexo e sua solução é dada por $\lambda^{*}$ tal que $\nabla q\left(\lambda^{*}\right)=0$. Assim sendo, podemos encontrar $\lambda^{*}$ resolvendo-se o seguinte sistema de equações lineares:

$$
A\left(A^{T} \lambda+c\right)=0 .
$$

Tomando $\beta=A^{T} \lambda+c$, podemos reescrever o sistema (3.15), obtendo

$$
\left\{\begin{aligned}
A^{T} \lambda-\beta & =-c \\
A \beta & =0
\end{aligned}\right.
$$

ou, matricialmente,

$$
\left(\begin{array}{cc}
-I & A^{T} \\
A & 0
\end{array}\right)\left(\begin{array}{l}
\beta \\
\lambda
\end{array}\right)=-\left(\begin{array}{l}
c \\
0
\end{array}\right)
$$

Notemos que a matriz dos coeficientes do sistema (3.16) é simétrica e esparsa, o que torna o sistema mais barato de ser resolvido e nos permite usar uma rotina apropriada. Após obtida a solução $\left(\beta^{*}, \lambda^{*}\right), \beta^{*}$ é descartado e caso $\lambda^{*}$ seja muito grande, isto é, se $\left\|\lambda^{*}\right\|_{\infty}>\lambda_{\max }$ (utilizaremos $\lambda_{\max }=10^{3}$ em nossa implementação), descartamos a solução encontrada e tomamos $\lambda=0$ como nossa estimativa inicial. A solução pode assumir valores muito altos se algumas linhas de $A$ forem quase linearmente dependentes. Em [25, p. 44], Wächter e Biegler propõem a inicialização dos multiplicadores associados às restrições de caixa como 1. Embora pareça mais natural inicializá-los com os valores definidos em (2.7), os valores propostos podem fazer com que o sistema (3.16) seja numericamente mais estável, e as correções eventualmente aplicadas usando (2.20) e (2.21) fazem com que os valores obtidos durante a otimização permaneçam dentro de um intervalo aceitável.

\subsection{Lidando com direções de busca pequenas}

Em algumas situações, pode acontecer que a direção $d_{x}^{k, j}$ encontrada seja muito pequena com relação ao tamanho de $x^{k, j}$. Isso pode ocorrer quando $x^{k, j}$ está próximo da solução, mas $\lambda^{k, j}, z_{\ell}^{k, j} \mathrm{e}$ $z_{u}^{k, j}$ não estão. Nesse caso, em primeiro lugar pode ser que o procedimento de backtracking não seja bem sucedido. Diante disso, a primeira medida consiste em permitir que $\alpha_{k, j}=\alpha_{k, j}^{\max }$ sempre que

$$
\max _{1 \leq i \leq n}\left\{\frac{\left|\left(d_{x}^{k, j}\right)_{i}\right|}{\left(1+\left|x_{i}^{k, j}\right|\right)}\right\}<10 \varepsilon_{\text {mach }}
$$


$\operatorname{com} \varepsilon_{\text {mach }}$ sendo uma aproximação da precisão da máquina (em nossa implementação, $\varepsilon_{\text {mach é um }}$ valor da ordem de $10^{-16}$ ). No caso disso ocorrer duas vezes consecutivas, o algoritmo considera que o subproblema de barreira foi resolvido e atualiza o parâmetro de barreira como será mostrado adiante. Caso o valor do parâmetro de barreira seja muito pequeno $\left(\varepsilon_{\text {tol }} / 10\right)$, o algoritmo decreta falha de convergência.

Além disso, pode ser necessário dar passo unitário nas variáveis $z_{\ell}$ e $z_{u}$ quando $d_{x}^{k, j}$ for suficientemente pequeno (veja a análise de convergência global do método na Seção 2.4.2 adiante). Para que $\alpha_{k, j}^{z_{\ell}}=1$ satisfaça (2.18c), é necessário que

$$
\left[z_{\ell}^{k, j}\right]_{i}+\left[d_{z_{\ell}}^{k, j}\right]_{i} \geq(1-\tau)\left[z_{\ell}^{k, j}\right]_{i} \text {, para } i \in \mathcal{I}_{\ell} \cap \mathcal{D}_{+}^{k, j} .
$$

De (2.13a) vem que

$$
\left[z_{\ell}^{k, j}\right]_{i}-\left[z_{\ell}^{k, j}\right]_{i}+\left[\mu_{k} L_{k, j}^{\dagger} e\right]_{i}-\left[L_{k, j}^{\dagger} Z_{k, j}^{\ell} d_{x}^{k, j}\right]_{i} \geq(1-\tau)\left[z_{\ell}^{k, j}\right]_{i}, \text { para } i \in \mathcal{I}_{\ell} \cap \mathcal{D}_{+}^{k, j} .
$$

Por conseguinte,

$$
\mu_{k}-\left[z_{\ell}^{k, j}\right]_{i}\left[d_{x}^{k, j}\right]_{i} \geq(1-\tau)\left[L_{k, j} z_{\ell}^{k, j}\right]_{i}, \text { para } i \in \mathcal{I}_{\ell} \cap \mathcal{D}_{+}^{k, j} .
$$

Como $x_{i} \geq \ell_{i}$, temos que $(1-\tau)\left[L_{k, j} z_{\ell}^{k, j}\right]_{i} \geq 0$, o que implica que quando

$$
\left[d_{x}^{k, j}\right]_{i} \leq \frac{\mu_{k}}{\left[z_{\ell}^{k, j}\right]_{i}}, \text { para } i \in \mathcal{I}_{\ell} \cap \mathcal{D}_{+}^{k, j},
$$

a condição de (2.18c) é satisfeita. De maneira semelhante, para que $\alpha_{k, j}^{z_{u}}=1$ satisfaça (2.18d), é necessário que

$$
\left[z_{u}^{k, j}\right]_{i}+\left[d_{z_{u}}^{k, j}\right]_{i} \geq(1-\tau)\left[z_{u}^{k, j}\right]_{i}, \text { para } i \in \mathcal{I}_{u} \cap \mathcal{D}_{-}^{k, j}
$$

De (2.13b) vem que

$$
\left[z_{u}^{k, j}\right]_{i}-\left[z_{u}^{k, j}\right]_{i}+\left[\mu_{k} U_{k, j}^{\dagger} e\right]_{i}+\left[U_{k, j}^{\dagger} Z_{k, j}^{u} d_{x}^{k, j}\right]_{i} \geq(1-\tau)\left[z_{u}^{k, j}\right]_{i}, \text { para } i \in \mathcal{I}_{u} \cap \mathcal{D}_{-}^{k, j} .
$$

Por conseguinte,

$$
\mu_{k}+\left[z_{u}^{k, j}\right]_{i}\left[d_{x}^{k, j}\right]_{i} \geq(1-\tau)\left[U_{k, j} z_{u}^{k, j}\right]_{i}, \text { para } i \in \mathcal{I}_{u} \cap \mathcal{D}_{-}^{k, j} .
$$

Como $x_{i} \leq u_{i}$, temos que $(1-\tau)\left[U_{k, j} z_{u}^{k, j}\right]_{i} \geq 0$, o que implica que quando

$$
\left[d_{x}^{k, j}\right]_{i} \geq-\frac{\mu_{k}}{\left[z_{u}^{k, j}\right]_{i}}, \text { para } i \in \mathcal{I}_{u} \cap \mathcal{D}_{-}^{k, j},
$$

a condição de (2.18d) é satisfeita. Assim sendo, $\alpha^{z_{\ell}}=\alpha^{z_{u}}=1$ satisfazem as condições de (2.18c) e (2.18d) sempre que

$$
\begin{aligned}
& {\left[d_{x}^{k, j}\right]_{i} \leq \frac{\mu_{k}}{\left[z_{\ell}^{k, j}\right]_{i}}, \text { para todo } i \in \mathcal{I}_{\ell} \cap \mathcal{D}_{+}^{k, j} \mathrm{e}} \\
& {\left[d_{x}^{k, j}\right]_{i} \geq-\frac{\mu_{k}}{\left[z_{u}^{k, j}\right]_{i}}, \text { para todo } i \in \mathcal{I}_{u} \cap \mathcal{D}_{-}^{k, j}}
\end{aligned}
$$

\subsection{Correção de inércia}

O valor de $\xi_{k, j}$ deve ser suficientemente grande para que $M_{k, j}$ possua a inércia desejada. Por outro lado, deve ser o menor possível, de forma que o sistema (2.14) seja o menos perturbado possível em (2.31). Diante disto, a principal ideia é encontrar um valor que ajuste a inércia da matriz o mais rápido possível, para assim evitar fatorações desnecessárias da matriz do sistema (2.31), e 
que seja o menor possível, para que o erro da solução encontrada de (2.31) com relação à solução de (2.14) não interfira na convergência do algoritmo. Não obstante, há problemas em que a matriz do sistema (2.14) pode ser estruturalmente singular, o que implica correção de inércia em todas as iterações. Deste modo, haverá uma fatoração desnecessária em todas iterações, o que é indesejável. Para evitar este tipo de situação, se em três iterações seguidas tivermos uma perturbação com $\xi_{k, j}>0$, a matriz do sistema (2.14) não será mais fatorada, mas somente a matriz do sistema (2.31) para algum $\xi_{k, j}>0$. Segue, no Algoritmo 3, o método para encontrar um valor para $\xi_{k, j}$.

Algoritmo 3. Cálculo de $\xi_{k, j}-$ correção de inércia.

Entrada: Constantes $0<\xi_{\min }<\xi_{\max } ; 0<\kappa_{\xi}^{-}<1<\kappa_{\xi}^{+}<\bar{\kappa}_{\xi}^{+} ; \xi_{\text {ini }}>0, \xi_{k, j-1}$ a correção anterior, para $j>1$, ou $\xi_{0,-1}=0$, para $j=0$.

Passo 1. $\xi_{k, j} \leftarrow 0$.

Passo 2. Se a inércia da matriz do sistema (2.14) for $(n, m, 0)$, faça $\xi_{k+1,-1}=\xi_{k, j}$ e pare.

Passo 3. Se $\xi_{k, j-1}=0$, então $\xi_{k, j} \leftarrow \xi_{\text {ini }}$, senão $\xi_{k, j} \leftarrow \max \left\{\xi_{\min }, \kappa_{\xi}^{-} \xi_{k, j-1}\right\}$.

Passo 4. Se a inércia da matriz do sistema (2.31) for $(n, m, 0)$, faça $\xi_{k+1,-1}=\xi_{k, j}$ e pare.

Passo 5. Se $\xi_{k, j-1}=0$, então $\xi_{k, j} \leftarrow \bar{\kappa}_{\xi}^{+} \xi_{k, j}$, senão $\xi_{k, j} \leftarrow \kappa_{\xi}^{+} \xi_{k, j}$.

Passo 6. Se $\xi_{k, j}>\xi_{\max }$, então pare, não foi possível computar um valor para $\xi_{k, j}$. Senão, vá para o Passo 4.

Notemos que, no Algoritmo 3, consideramos o valor $\xi_{k, j-1}$ usado na correção anterior, de forma que $\xi_{0,-1}=0$ no início de tudo e, para $k>0, \xi_{k,-1}$ o último valor usado na $(k-1)$-ésima iteração. Além disso, definimos constantes $0<\xi_{\min }<\xi_{\max }$ de maneira que os valores não nulos que $\xi_{k, j}$ assuma estejam no intervalo $\left[\xi_{\min }, \xi_{\max }\right]$, a fim de evitar valores insignificantes ou valores demasiadamente grandes. Finalmente, consideramos constantes $0<\kappa_{\xi}^{-}<1<\kappa_{\xi}^{+}<\bar{\kappa}_{\xi}^{+}$que controlam a variação de $\xi_{k, j}$ durante uma correção e uma constante $\xi_{\text {ini }}>0$, que é o valor inicial de $\xi_{k, j}$ para uma correção. Na prática, tomamos $\xi_{\text {ini }}=10^{-4}, \xi_{\min }=10^{-20}, \xi_{\max }=10^{20}, \kappa_{\xi}^{-}=1 / 3, \kappa_{\xi}^{+}=8$ e $\bar{\kappa}_{\xi}^{+}=100$. 


\section{Capítulo 4}

\section{Experimentos numéricos}

Os testes foram efetuados em um Intel(R) Xeon(R) CPU X5650 2.67GHz, com 8 GB de memória RAM e sistema operacional Ubuntu 12.04.1 LTS. O código foi compilado usando-se o compilador gfortran versão 4.6.3.

Os valores de constantes usados na implementação foram descritos ao longo deste texto. Ademais, tomamos 200 como sendo a quantidade máxima de iterações internas e 50 como sendo a quantidade máxima de iterações externas. Como critério de parada adicional, consideramos que se a norma infinito do iterando corrente fosse suficientemente grande (neste caso, consideramos maior ou igual a $10^{20}$ ), então paramos e decretamos que os iterandos divergiram, o que é um indício de que a função objetivo do problema é ilimitada.

Dividimos os experimentos em duas partes. Na primeira, exibimos os resultados da execução de LCMIN para problemas do CUTEst. Na segunda parte, para o mesmo conjunto de problemas, fazemos uma análise comparativa entre ALGENCAN, IPOPT e LCMIN.

\subsection{Parte I - Resultados gerais}

Numa primeira etapa, consideramos todos os problemas da versão atual do pacote CUTEst [17] que possuem restrições lineares de igualdade e de caixa, totalizando 200 problemas. Para cada problema, consideramos:

\begin{tabular}{c|l} 
Notação & Descrição \\
\hline \hline prob & o nome do problema no pacote CUTEst \\
\hline $\mathrm{cp}$ & o critério de parada do algoritmo \\
\hline $\mathrm{n}$ & a quantidade de variáveis \\
\hline $\mathrm{m}$ & a quantidade de restrições (lineares de igualdade) \\
\hline$f\left(x^{*}\right)$ & o valor da função objetivo na solução encontrada, não escalado \\
\hline$\left\|A x^{*}-b\right\|$ & a viabilidade da solução com relação às restrições lineares, não escalada \\
\hline$E_{0}$ & o erro de otimalidade, escalado \\
\hline$\# \mathrm{f}$ & a quantidade de avaliações da função objetivo \\
\hline$\#$ iti & a quantidade de iterações internas totais \\
\hline$\#$ ite & a quantidade de iterações externas \\
\hline ppt & tempo de pré-processamento (em segundos) \\
\hline opt & tempo de otimização (em segundos) \\
\hline
\end{tabular}


O tempo de otimização consiste na execução do método apresentado neste trabalho, isto é, dado um ponto inicial estritamente viável, o tempo dispendido para executar o algoritmo apresentado no Capítulo 2. O tempo de pré-processamento, por sua vez, é consumido principalmente pelo cálculo de um ponto inicial estritamente viável. Na Seção 3.3, apresentamos duas estratégias para encontrar um ponto inicial estritamente viável: uma que envolve a resolução de um problema de programação linear (veja Seção 3.3.1) e outra que consiste em resolver um problema de viabilidade (veja Seção 3.3.2). Na prática, a estratégia que usa programação linear mostrou-se robusta, pois de fato ou encontra um ponto inicial estritamente viável ou conclui que um tal ponto não existe. Todavia é ineficiente, pois consome um tempo de execução impraticável. A estratégia que envolve problemas de viabilidade mostrou-se melhor na prática, por consumir um menor tempo de execução, e agregou competitividade ao método proposto. Desta forma, o tempo de pré-processamento é, essencialmente, o tempo que ALGENCAN demora para resolver o problema de viabilidade apresentado na Seção 3.3.2.

A cada critério de parada associamos um número, a saber:

0 : as condições de otimalidade do problema foram satisfeitas

1: LCMIN convergiu a um ponto quase viável

-1: máximo de iterações externas excedido

-3 : falta de progresso por máximo de iterações internas

$-4:$ os iterandos divergiram

-6 : o conjunto viável é vazio

-50 : tempo máximo de execução excedido.

Sobre cada critério, cabe aqui um breve comentário. LCMIN para com saída 0 quando um ponto $\left(\bar{x}, \bar{\lambda}, \bar{z}_{\ell}, \bar{z}_{u}\right)$ satisfizer $(3.3) \mathrm{e}$

$$
\|A \bar{x}-b\|_{\infty} \leq \varepsilon_{\mathrm{v}},
$$

$\operatorname{com} \varepsilon_{\mathrm{v}}>0$ uma tolerância de viabilidade (nos experimentos, $\varepsilon_{\mathrm{v}}=10^{-8}$ ). Todavia, por problemas numéricos, pode ser que o LCMIN falhe em conservar a viabilidade do ponto inicial durante a otimização e que (4.1) não seja satisfeita no ponto final, ainda que (3.3) seja. Nestes casos, se a viabilidade do ponto final satisfizer

$$
\|A \bar{x}-b\|_{\infty} \leq \sqrt{\varepsilon_{\mathrm{v}}},
$$

LCMIN para com saída 1, caso contrário, declara que convergiu a um ponto inviável. Finalmente, para cada problema estabelecemos um tempo limite de duas horas para execução do LCMiN; caso ele não termine a execução dentro deste tempo, denotamos a parada pela saída -50 . Os demais critérios de parada podem ser vistos com detalhes na Seção 3.1.

Para a resolução dos sistemas lineares envolvidos na fase de otimização, utilizamos a rotina HSL MA57 da Harwell Subroutine Library [19], bem como na execução de ALGENCAN para a computação do ponto inicial.

Os resultados dos testes são expostos na Tabela 4.1.

\begin{tabular}{|c|c|c|c|c|c|c|c|c|c|c|c|}
\hline prob & $\mathrm{cp}$ & $\mathrm{n}$ & $\mathrm{m}$ & $f\left(x^{*}\right)$ & $A x^{*}-b$ & $E_{0}$ & $\# \mathrm{f}$ & $\#$ iti & $\#$ ite & ppt & opt \\
\hline A0ENDNDL & 0 & 45006 & 15002 & $1.25313 \mathrm{D}-05$ & $3.0569 \mathrm{D}-12$ & $2.5101 \mathrm{D}-09$ & 6 & 5 & 6 & 0.46 & 0.34 \\
\hline A0ENINDL & 0 & 45006 & 15002 & $1.25312 \mathrm{D}-05$ & $1.2194 \mathrm{D}-13$ & $2.5098 \mathrm{D}-09$ & 6 & 5 & 6 & 0.61 & 0.34 \\
\hline A0ENSNDL & 0 & 45006 & 15002 & $1.00626 \mathrm{D}-05$ & $5.0093 \mathrm{D}-13$ & $3.2958 \mathrm{D}-09$ & 7 & 5 & 6 & 0.38 & 0.96 \\
\hline A0ESDNDL & 0 & 45006 & 15002 & $1.25312 \mathrm{D}-05$ & $9.7983 \mathrm{D}-12$ & $2.5096 \mathrm{D}-09$ & 6 & 5 & 6 & 0.42 & 0.34 \\
\hline A0ESINDL & 0 & 45006 & 15002 & $1.25312 \mathrm{D}-05$ & $1.9389 \mathrm{D}-12$ & $2.5097 \mathrm{D}-09$ & 6 & 5 & 6 & 0.57 & 0.34 \\
\hline A0ESSNDL & 0 & 45006 & 15002 & $1.00626 \mathrm{D}-05$ & $2.3054 \mathrm{D}-11$ & $3.2962 \mathrm{D}-09$ & 7 & 5 & 6 & 0.38 & 1.05 \\
\hline A0NNDNDL & 0 & 60012 & 20004 & $1.25320 \mathrm{D}-05$ & $2.2112 \mathrm{D}-12$ & $2.5386 \mathrm{D}-09$ & 26 & 25 & 6 & 4.40 & 2.01 \\
\hline A0NNDNIL & 0 & 60012 & 20004 & $1.06733 \mathrm{D}-01$ & $6.5267 \mathrm{D}-13$ & $1.0082 \mathrm{D}-09$ & 76 & 75 & 7 & 52.43 & 8.06 \\
\hline
\end{tabular}

Tabela 4.1: Resultados dos experimentos com 200 problemas do pacote CUTEst. 


\begin{tabular}{|c|c|c|c|c|c|c|c|c|c|c|c|}
\hline prob & $\mathrm{cp}$ & $\mathrm{n}$ & $\mathrm{m}$ & $f\left(x^{*}\right)$ & $\left\|A x^{*}-b\right\|$ & $E_{0}$ & $\# \mathrm{f}$ & $\#$ iti & $\#$ ite & ppt & opt \\
\hline A0NNDNSL & 0 & 60012 & 20004 & $4.51700 \mathrm{D}-06$ & $1.0164 \mathrm{D}-10$ & $1.0000 \mathrm{D}-09$ & 20 & 19 & 7 & 3.00 & 2.12 \\
\hline A0NNSNSL & 0 & 60012 & 20004 & $9.99034 \mathrm{D}-06$ & $6.4233 \mathrm{D}-12$ & $2.8962 \mathrm{D}-09$ & 17 & 16 & 6 & 4.25 & 1.54 \\
\hline A0NSDSDL & 0 & 60012 & 20004 & $1.25319 \mathrm{D}-05$ & $1.9336 \mathrm{D}-12$ & $2.5493 \mathrm{D}-09$ & 17 & 16 & 6 & 6.55 & 1.32 \\
\hline A0NSDSDS & 0 & 6012 & 2004 & $3.57333 \mathrm{D}-07$ & $8.8107 \mathrm{D}-13$ & $1.1985 \mathrm{D}-09$ & 20 & 19 & 7 & 0.44 & 0.18 \\
\hline A0NSDSIL & 0 & 60012 & 20004 & $2.73647 \mathrm{D}+01$ & $1.8190 \mathrm{D}-12$ & $4.9532 \mathrm{D}-09$ & 207 & 189 & 6 & 27.43 & 21.35 \\
\hline A0NSDSSL & 0 & 60012 & 20004 & $1.13191 \mathrm{D}-05$ & $1.0216 \mathrm{D}-09$ & $2.5227 \mathrm{D}-09$ & 11944 & 218 & 6 & 9.67 & 33.89 \\
\hline A0NSSSSL & 0 & 60012 & 20004 & $9.98975 \mathrm{D}-06$ & $3.0850 \mathrm{D}-11$ & $3.7314 \mathrm{D}-09$ & 20 & 19 & 6 & 4.28 & 1.68 \\
\hline A2ENDNDL & 0 & 45006 & 15002 & $1.83609 \mathrm{D}-05$ & $9.2371 \mathrm{D}-13$ & $8.4261 \mathrm{D}-09$ & 14 & 13 & 6 & 0.76 & 0.86 \\
\hline A2ENINDL & 0 & 45006 & 15002 & $7.48038 \mathrm{D}-06$ & $9.1660 \mathrm{D}-13$ & $4.4774 \mathrm{D}-09$ & 15 & 14 & 7 & 0.59 & 0.82 \\
\hline A2ENSNDL & 0 & 45006 & 15002 & $5.35274 \mathrm{D}-06$ & $2.4922 \mathrm{D}-11$ & $4.0528 \mathrm{D}-09$ & 17 & 16 & 7 & 0.49 & 1.74 \\
\hline A2ESDNDL & 0 & 45006 & 15002 & $1.83609 \mathrm{D}-05$ & $2.5935 \mathrm{D}-13$ & $8.4261 \mathrm{D}-09$ & 14 & 13 & 6 & 0.74 & 0.86 \\
\hline A2ESINDL & 0 & 45006 & 15002 & $7.35681 \mathrm{D}-06$ & $7.6277 \mathrm{D}-12$ & $4.4731 \mathrm{D}-09$ & 15 & 14 & 7 & 0.41 & 0.82 \\
\hline A2ESSNDL & 0 & 45006 & 15002 & $5.44632 \mathrm{D}-06$ & $1.0658 \mathrm{D}-12$ & $4.2235 \mathrm{D}-09$ & 17 & 16 & 7 & 0.64 & 1.66 \\
\hline A2NNDNDL & 0 & 60012 & 20004 & $1.83416 \mathrm{D}-05$ & $1.7057 \mathrm{D}-12$ & $9.6343 \mathrm{D}-09$ & 22 & 21 & 6 & 10.45 & 1.77 \\
\hline A2NNDNIL & 1 & 60012 & 20004 & $7.08548 \mathrm{D}+00$ & $1.1740 \mathrm{D}-05$ & $6.4299 \mathrm{D}-09$ & 119 & 95 & 6 & 61.76 & 12.67 \\
\hline A2NNDNSL & 0 & 60012 & 20004 & $1.10534 \mathrm{D}-05$ & $2.9366 \mathrm{D}-10$ & $3.9495 \mathrm{D}-09$ & 40 & 32 & 6 & 12.69 & 3.72 \\
\hline A2NNSNSL & 0 & 60012 & 20004 & $3.83250 \mathrm{D}-06$ & $1.1120 \mathrm{D}-12$ & $3.7475 \mathrm{D}-09$ & 23 & 22 & 7 & 4.98 & 1.88 \\
\hline A2NSDSDL & 0 & 60012 & 20004 & $7.26269 \mathrm{D}-06$ & $2.9559 \mathrm{D}-12$ & $5.8601 \mathrm{D}-09$ & 24 & 23 & 7 & 8.61 & 1.87 \\
\hline A2NSDSIL & 1 & 60012 & 20004 & $1.26186 \mathrm{D}+02$ & $3.6417 \mathrm{D}-06$ & $3.1222 \mathrm{D}-09$ & 119 & 108 & 6 & 94.55 & 14.53 \\
\hline A2NSDSSL & 0 & 60012 & 20004 & $1.10553 \mathrm{D}-05$ & $4.0927 \mathrm{D}-12$ & $6.8942 \mathrm{D}-09$ & 32 & 30 & 6 & 18.81 & 3.60 \\
\hline A2NSSSSL & 0 & 60012 & 20004 & $3.82509 \mathrm{D}-06$ & $3.4687 \mathrm{D}-12$ & $1.1431 \mathrm{D}-09$ & 28 & 27 & 7 & 15.49 & 2.32 \\
\hline A5ENDNDL & 0 & 45006 & 15002 & $2.70980 \mathrm{D}-05$ & $2.8066 \mathrm{D}-13$ & $8.4261 \mathrm{D}-09$ & 14 & 13 & 6 & 1.48 & 0.96 \\
\hline A5ENINDL & -1 & 45006 & 15002 & $3.04825 \mathrm{D}-05$ & $2.0606 \mathrm{D}-13$ & $1.0541 \mathrm{D}-08$ & 14 & 13 & 51 & 1.34 & 0.94 \\
\hline A5ENSNDL & 0 & 45006 & 15002 & $1.73184 \mathrm{D}-05$ & $1.7302 \mathrm{D}-12$ & $8.8555 \mathrm{D}-09$ & 16 & 15 & 6 & 0.67 & 1.71 \\
\hline A5ESDNDL & 0 & 45006 & 15002 & $2.70991 \mathrm{D}-05$ & $6.0751 \mathrm{D}-13$ & $8.4261 \mathrm{D}-09$ & 14 & 13 & 6 & 1.00 & 0.95 \\
\hline A5ESINDL & 0 & 45006 & 15002 & $2.75017 \mathrm{D}-05$ & $4.3698 \mathrm{D}-13$ & $8.4261 \mathrm{D}-09$ & 14 & 13 & 6 & 1.21 & 1.12 \\
\hline A5ESSNDL & 0 & 45006 & 15002 & $1.19796 \mathrm{D}-05$ & $2.4567 \mathrm{D}-11$ & $7.0915 \mathrm{D}-09$ & 16 & 15 & 7 & 0.72 & 1.60 \\
\hline A5NNDNDL & 0 & 60012 & 20004 & $1.25184 \mathrm{D}-05$ & $3.9790 \mathrm{D}-13$ & $3.1557 \mathrm{D}-09$ & 59 & 46 & 6 & 15.20 & 4.43 \\
\hline A5NNDNIL & 1 & 60012 & 20004 & $4.25802 \mathrm{D}+00$ & $1.1548 \mathrm{D}-05$ & $5.0026 \mathrm{D}-09$ & 120 & 108 & 6 & 43.47 & 14.25 \\
\hline A5NNDNSL & 0 & 60012 & 20004 & $1.06627 \mathrm{D}-05$ & $8.0030 \mathrm{D}-13$ & $2.5570 \mathrm{D}-09$ & 49 & 33 & 6 & 8.47 & 4.16 \\
\hline A5NNSNSL & 0 & 60012 & 20004 & $9.03680 \mathrm{D}-06$ & $8.5606 \mathrm{D}-11$ & $8.4236 \mathrm{D}-09$ & 38 & 26 & 6 & 5.72 & 2.30 \\
\hline A5NSDSDL & 0 & 60012 & 20004 & $1.25415 \mathrm{D}-05$ & $6.0002 \mathrm{D}-13$ & $4.7992 \mathrm{D}-09$ & 20 & 19 & 7 & 12.26 & 1.54 \\
\hline A5NSDSDM & 0 & 6012 & 2004 & $3.57333 \mathrm{D}-07$ & $8.8107 \mathrm{D}-13$ & $1.1985 \mathrm{D}-09$ & 20 & 19 & 7 & 0.43 & 0.18 \\
\hline A5NSDSIL & 1 & 60012 & 20004 & $3.17919 \mathrm{D}+00$ & $3.7736 \mathrm{D}-06$ & $2.0597 \mathrm{D}-09$ & 114 & 111 & 7 & 67.27 & 14.51 \\
\hline A5NSDSSL & 0 & 60012 & 20004 & $4.28695 \mathrm{D}-06$ & $1.3713 \mathrm{D}-12$ & $3.8108 \mathrm{D}-09$ & 24 & 23 & 7 & 6.20 & 2.68 \\
\hline A5NSSNSM & 0 & 6012 & 2004 & $3.57333 \mathrm{D}-07$ & $8.8107 \mathrm{D}-13$ & $1.1985 \mathrm{D}-09$ & 20 & 19 & 7 & 0.43 & 0.18 \\
\hline A5NSSSSL & 0 & 60012 & 20004 & $3.60794 \mathrm{D}-06$ & $1.2132 \mathrm{D}-11$ & $5.0814 \mathrm{D}-09$ & 29 & 28 & 7 & 5.07 & 2.34 \\
\hline ARGLALE & -6 & 200 & 400 & - & - & - & - & - & - & 0.09 & - \\
\hline ARGLBLE & -6 & 200 & 400 & - & - & - & - & - & - & 0.01 & - \\
\hline ARGLCLE & -6 & 200 & 399 & - & - & - & - & - & - & 0.01 & - \\
\hline AUG2DCQP & 0 & 20200 & 10000 & $6.49813 \mathrm{D}+06$ & $6.3949 \mathrm{D}-14$ & $2.7143 \mathrm{D}-09$ & 20 & 19 & 6 & 5.06 & 1.14 \\
\hline AUG2DC & 0 & 20200 & 10000 & $1.81836 \mathrm{D}+06$ & $4.3485 \mathrm{D}-10$ & $4.3362 \mathrm{D}-14$ & 2 & 1 & 1 & 0.44 & 0.19 \\
\hline AUG2DQP & 0 & 20200 & 10000 & $6.23701 \mathrm{D}+06$ & $1.6776 \mathrm{D}-11$ & $3.6609 \mathrm{D}-09$ & 22 & 21 & 7 & 4.66 & 1.42 \\
\hline AUG2D & 0 & 20200 & 10000 & $1.68741 \mathrm{D}+06$ & $4.3485 \mathrm{D}-10$ & $3.9240 \mathrm{D}-09$ & 3 & 2 & 1 & 0.44 & 0.42 \\
\hline AUG3DCQP & 0 & 27543 & 8000 & $6.15603 \mathrm{D}+04$ & $3.5663 \mathrm{D}-10$ & $4.0302 \mathrm{D}-09$ & 20 & 19 & 7 & 1.08 & 10.90 \\
\hline AUG3DC & 0 & 27543 & 8000 & $2.76540 \mathrm{D}+04$ & $4.0591 \mathrm{D}-10$ & $4.8850 \mathrm{D}-15$ & 2 & 1 & 1 & 0.27 & 1.27 \\
\hline AUG3DQP & 0 & 27543 & 8000 & $5.42289 \mathrm{D}+04$ & $3.5663 \mathrm{D}-10$ & $5.7081 \mathrm{D}-09$ & 19 & 18 & 7 & 1.08 & 10.07 \\
\hline AUG3D & 0 & 27543 & 8000 & $2.45614 \mathrm{D}+04$ & $4.0591 \mathrm{D}-10$ & $7.6285 \mathrm{D}-09$ & 3 & 2 & 1 & 0.27 & 16.36 \\
\hline AVION2 & -3 & 49 & 15 & $9.46802 \mathrm{D}+07$ & $5.7980 \mathrm{D}-12$ & $5.5147 \mathrm{D}-05$ & 672 & 610 & 6 & 0.00 & 0.21 \\
\hline BDRY2 & -50 & 251001 & 250498 & - & - & - & - & - & - & 7200.00 & - \\
\hline BIGBANK & 0 & 2230 & 1112 & $-4.20569 \mathrm{D}+06$ & $4.0927 \mathrm{D}-11$ & $5.8550 \mathrm{D}-09$ & 37 & 28 & 7 & 0.10 & 0.17 \\
\hline BLOWEYA & -1 & 4002 & 2002 & $-2.27810 \mathrm{D}-02$ & $3.9245 \mathrm{D}-10$ & $1.0386 \mathrm{D}-08$ & 11 & 10 & 51 & 1.73 & 0.34 \\
\hline BLOWEYB & 0 & 4002 & 2002 & $-1.52257 \mathrm{D}-02$ & $9.3514 \mathrm{D}-10$ & $1.0465 \mathrm{D}-09$ & 9 & 8 & 7 & 0.47 & 0.29 \\
\hline BLOWEYC & 0 & 4002 & 2002 & $-1.52457 \mathrm{D}-02$ & $2.2233 \mathrm{D}-10$ & $2.9956 \mathrm{D}-09$ & 13 & 12 & 6 & 0.98 & 0.39 \\
\hline BOOTH & 0 & 2 & 2 & $0.00000 \mathrm{D}+00$ & $3.5527 \mathrm{D}-15$ & $0.0000 \mathrm{D}+00$ & 1 & 0 & 0 & 0.00 & 0.00 \\
\hline BT3 & 0 & 5 & 3 & $4.09302 \mathrm{D}+00$ & $1.7764 \mathrm{D}-15$ & $5.8287 \mathrm{D}-15$ & 2 & 1 & 1 & 0.00 & 0.00 \\
\hline BTS4 & 0 & 36570 & 36310 & $1.60921 \mathrm{D}+04$ & $4.4226 \mathrm{D}-09$ & $4.1062 \mathrm{D}-09$ & 23 & 22 & 7 & 3.96 & 13.51 \\
\hline $\mathrm{CBS}$ & 0 & 11163 & 244 & $8.37610 \mathrm{D}+04$ & $2.6319 \mathrm{D}-11$ & $1.0954 \mathrm{D}-09$ & 27 & 26 & 7 & 0.24 & 0.72 \\
\hline CONT5-QP & -50 & 40601 & 40200 & - & - & - & - & - & - & 7200.00 & - \\
\hline CVXQP1 & 0 & 10000 & 5000 & $1.08704 \mathrm{D}+08$ & $4.3885 \mathrm{D}-12$ & $9.0124 \mathrm{D}-09$ & 19 & 18 & 6 & 2.78 & 59.40 \\
\hline CVXQP2 & 0 & 10000 & 2500 & $8.18424 \mathrm{D}+07$ & $2.3093 \mathrm{D}-14$ & $9.9463 \mathrm{D}-09$ & 21 & 20 & 6 & 0.10 & 20.58 \\
\hline CVXQP3 & 0 & 10000 & 7500 & $1.15711 \mathrm{D}+08$ & $2.9718 \mathrm{D}-10$ & $3.1857 \mathrm{D}-09$ & 31 & 25 & 6 & 3.23 & 132.50 \\
\hline DALE & 0 & 16514 & 405 & $3.70234 \mathrm{D}+03$ & $1.8794 \mathrm{D}-09$ & $1.0196 \mathrm{D}-09$ & 17 & 16 & 7 & 0.30 & 1.44 \\
\hline DALLASL & 0 & 906 & 667 & $-2.02604 \mathrm{D}+05$ & $3.3734 \mathrm{D}-09$ & $2.5059 \mathrm{D}-09$ & 29 & 28 & 6 & 0.12 & 0.12 \\
\hline DALLASM & 0 & 196 & 151 & $-4.81981 \mathrm{D}+04$ & $1.5010 \mathrm{D}-13$ & $2.5059 \mathrm{D}-09$ & 23 & 22 & 6 & 0.01 & 0.02 \\
\hline DALLASS & 0 & 46 & 31 & $-3.23932 \mathrm{D}+04$ & $2.4459 \mathrm{D}-09$ & $2.5064 \mathrm{D}-09$ & 28 & 27 & 6 & 0.00 & 0.01 \\
\hline DEGENLPA & 0 & 20 & 15 & $3.06039 \mathrm{D}+00$ & $1.7361 \mathrm{D}-10$ & $5.8028 \mathrm{D}-09$ & 14 & 12 & 7 & 0.08 & 0.00 \\
\hline DEGENLPB & 0 & 20 & 15 & $-3.07312 \mathrm{D}+01$ & $1.7361 \mathrm{D}-10$ & $2.5062 \mathrm{D}-09$ & 13 & 12 & 6 & 0.08 & 0.00 \\
\hline DEGTRIDL & 1 & 100001 & 1 & $5.00000 \mathrm{D}-01$ & $2.1090 \mathrm{D}-07$ & $4.6755 \mathrm{D}-09$ & 28 & 15 & 6 & 1.06 & 2.79 \\
\hline DTOC1L & 0 & 5998 & 3996 & $3.94304 \mathrm{D}+00$ & $1.6653 \mathrm{D}-16$ & $3.7571 \mathrm{D}-12$ & 10 & 9 & 1 & 0.03 & 0.18 \\
\hline DTOC3 & 0 & 4499 & 2998 & $2.35216 \mathrm{D}+02$ & $3.2954 \mathrm{D}-14$ & $2.4255 \mathrm{D}-14$ & 2 & 1 & 1 & 0.92 & 0.03 \\
\hline DUAL1 & 0 & 85 & 1 & $3.50129 \mathrm{D}-02$ & $8.6532 \mathrm{D}-10$ & $3.0938 \mathrm{D}-09$ & 20 & 18 & 7 & 0.00 & 0.03 \\
\hline DUAL2 & 0 & 96 & 1 & $3.37336 \mathrm{D}-02$ & $8.6736 \mathrm{D}-10$ & $1.3706 \mathrm{D}-09$ & 17 & 15 & 7 & 0.00 & 0.03 \\
\hline DUAL3 & 0 & 111 & 1 & $1.35755 \mathrm{D}-01$ & $1.1102 \mathrm{D}-15$ & $9.3269 \mathrm{D}-09$ & 22 & 16 & 6 & 0.00 & 0.04 \\
\hline DUAL4 & 0 & 75 & 1 & $7.46090 \mathrm{D}-01$ & $9.9920 \mathrm{D}-16$ & $4.3994 \mathrm{D}-09$ & 17 & 15 & 7 & 0.00 & 0.02 \\
\hline EXTRASIM & 0 & 2 & 1 & $1.00000 \mathrm{D}+00$ & $2.2204 \mathrm{D}-16$ & $2.5059 \mathrm{D}-09$ & 4 & 3 & 6 & 0.00 & 0.00 \\
\hline FCCU & 0 & 19 & 8 & $1.11491 \mathrm{D}+01$ & $7.1054 \mathrm{D}-15$ & $2.5785 \mathrm{D}-09$ & 9 & 8 & 6 & 0.00 & 0.00 \\
\hline FERRISDC & 0 & 2200 & 210 & $-2.29901 \mathrm{D}-04$ & $9.5146 \mathrm{D}-14$ & $3.1800 \mathrm{D}-09$ & 50 & 21 & 6 & 0.02 & 27.29 \\
\hline FIVE20B & 0 & 34552 & 52983 & $2.73533 \mathrm{D}+04$ & $4.0259 \mathrm{D}-11$ & $4.4039 \mathrm{D}-09$ & 19 & 18 & 6 & 30.88 & 122.22 \\
\hline
\end{tabular}




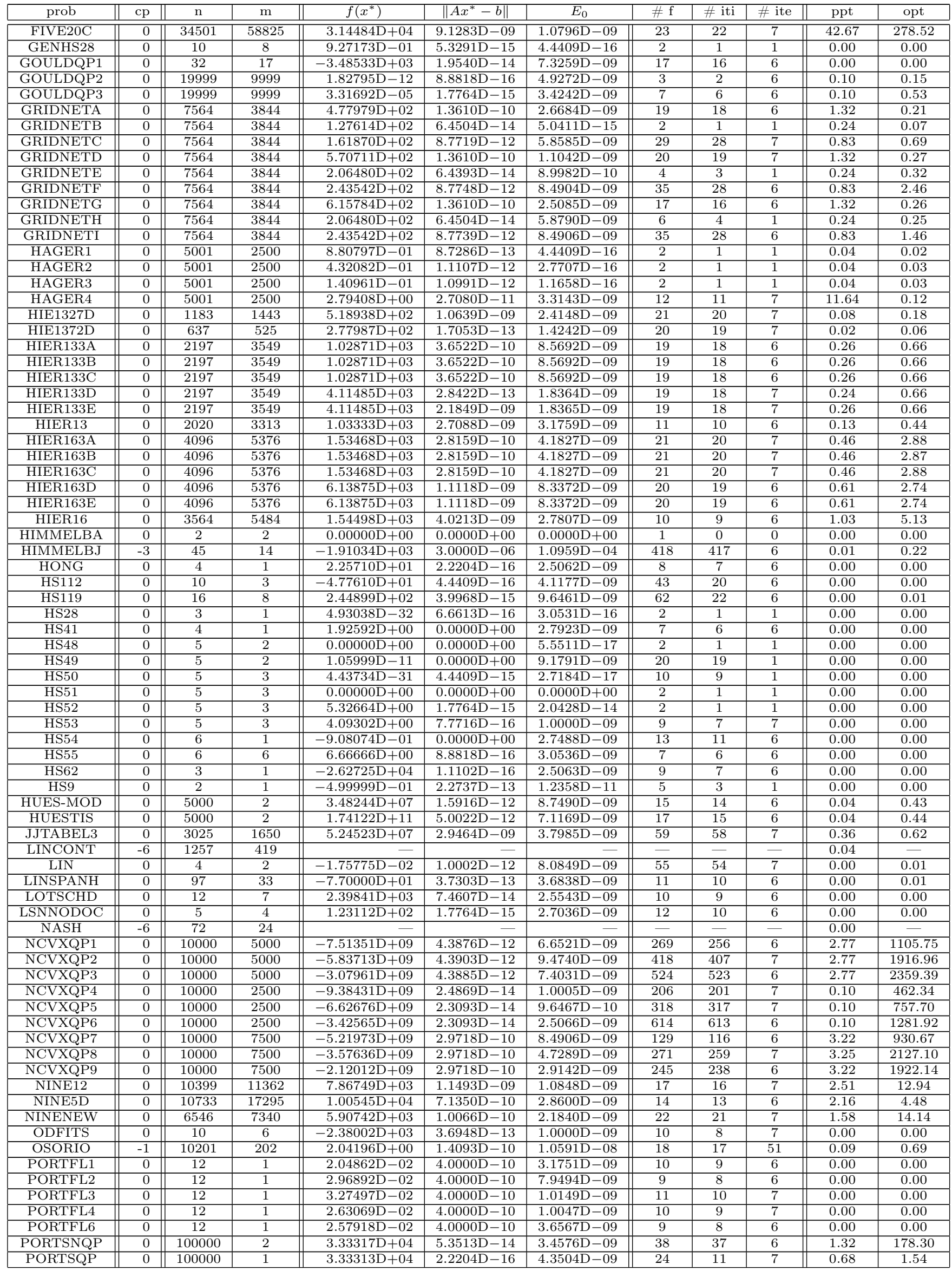




\begin{tabular}{|c|c|c|c|c|c|c|c|c|c|c|c|}
\hline prob & $\mathrm{cp}$ & $\mathrm{n}$ & $\mathrm{m}$ & $f\left(x^{*}\right)$ & $\left\|A x^{*}-b\right\|$ & $E_{0}$ & $\# \mathrm{f}$ & $\#$ iti & $\#$ ite & $\mathrm{ppt}$ & opt \\
\hline READING2 & 0 & 6003 & 4000 & $-1.25809 \mathrm{D}-02$ & $1.7251 \mathrm{D}-13$ & $2.0095 \mathrm{D}-09$ & 48 & 21 & 7 & 0.03 & 0.51 \\
\hline SMBANK & 0 & 117 & 64 & $-7.12929 \mathrm{D}+06$ & $8.7311 \mathrm{D}-11$ & $2.5078 \mathrm{D}-09$ & 17 & 16 & 6 & 0.00 & 0.01 \\
\hline SOSQP1 & 0 & 5000 & 2501 & $0.00000 \mathrm{D}+00$ & $0.0000 \mathrm{D}+00$ & $0.0000 \mathrm{D}+00$ & 1 & 0 & 0 & 0.02 & 0.00 \\
\hline SOSQP2 & 0 & 5000 & 2501 & $-1.24870 \mathrm{D}+03$ & $1.2733 \mathrm{D}-11$ & $4.5413 \mathrm{D}-09$ & 17 & 16 & 7 & 7.93 & 0.78 \\
\hline SPANHYD & 0 & 97 & 33 & $2.39738 \mathrm{D}+02$ & $5.3646 \mathrm{D}-13$ & $2.5059 \mathrm{D}-09$ & 18 & 17 & 6 & 0.00 & 0.01 \\
\hline STATIC3 & -4 & 434 & 96 & $-3.19792 \mathrm{D}+44$ & $9.6756 \mathrm{D}-14$ & $5.5559 \mathrm{D}+21$ & 1128 & 1127 & 6 & 0.01 & 1.74 \\
\hline STCQP1 & 0 & 8193 & 4095 & $3.67100 \mathrm{D}+05$ & $0.0000 \mathrm{D}+00$ & $2.5072 \mathrm{D}-09$ & 14 & 13 & 6 & 0.04 & 0.24 \\
\hline STCQP2 & 0 & 8193 & 4095 & $3.71892 \mathrm{D}+04$ & $0.0000 \mathrm{D}+00$ & $8.5577 \mathrm{D}-09$ & 14 & 13 & 6 & 0.04 & 0.34 \\
\hline STEENBRA & 0 & 432 & 108 & $1.69576 \mathrm{D}+04$ & $1.5518 \mathrm{D}-09$ & $3.4106 \mathrm{D}-09$ & 16 & 15 & 6 & 0.01 & 0.08 \\
\hline STEENBRB & 0 & 468 & 108 & $1.13557 \mathrm{D}+04$ & $4.6054 \mathrm{D}-10$ & $5.7610 \mathrm{D}-09$ & 121 & 80 & 7 & 0.00 & 0.47 \\
\hline STEENBRC & 0 & 540 & 126 & $3.77364 \mathrm{D}+04$ & $5.4570 \mathrm{D}-12$ & $5.8544 \mathrm{D}-09$ & 198 & 143 & 7 & 0.01 & 1.10 \\
\hline STEENBRD & 0 & 468 & 108 & $1.19530 \mathrm{D}+04$ & $7.5033 \mathrm{D}-12$ & $7.2081 \mathrm{D}-09$ & 347 & 136 & 7 & 0.01 & 0.81 \\
\hline STEENBRE & 0 & 540 & 126 & $3.64185 \mathrm{D}+04$ & $5.1409 \mathrm{D}-11$ & $8.9798 \mathrm{D}-09$ & 20540 & 481 & 7 & 0.01 & 4.02 \\
\hline STEENBRF & 0 & 468 & 108 & $1.19990 \mathrm{D}+04$ & $4.6049 \mathrm{D}-10$ & $8.8308 \mathrm{D}-09$ & 130 & 83 & 7 & 0.01 & 0.49 \\
\hline STEENBRG & 0 & 540 & 126 & $3.67799 \mathrm{D}+04$ & $5.0724 \mathrm{D}-12$ & $7.6182 \mathrm{D}-09$ & 221 & 142 & 7 & 0.01 & 1.09 \\
\hline STNQP1 & 0 & 8193 & 4095 & $-3.11704 \mathrm{D}+05$ & $0.0000 \mathrm{D}+00$ & $2.5063 \mathrm{D}-09$ & 15 & 14 & 6 & 0.04 & 0.28 \\
\hline STNQP2 & 0 & 8193 & 4095 & $-5.74969 \mathrm{D}+05$ & $0.0000 \mathrm{D}+00$ & $2.5062 \mathrm{D}-09$ & 17 & 16 & 6 & 0.04 & 0.51 \\
\hline SUPERSIM & 0 & 2 & 2 & $6.66666 \mathrm{D}-01$ & $0.0000 \mathrm{D}+00$ & $2.5059 \mathrm{D}-09$ & 1 & 4 & 6 & 0.00 & 0.00 \\
\hline SYNPOP24 & -6 & 6968 & 7353 & - & - & - & - & - & - & 0.02 & - \\
\hline TABLE1 & 0 & 1584 & 510 & $3.70607 \mathrm{D}+05$ & $6.4028 \mathrm{D}-10$ & $9.6468 \mathrm{D}-09$ & 22 & 21 & 6 & 0.05 & 0.11 \\
\hline TABLE3 & 0 & 4992 & 2464 & $4.36423 \mathrm{D}+04$ & $1.3642 \mathrm{D}-11$ & $1.6488 \mathrm{D}-09$ & 28 & 27 & 7 & 0.61 & 2.41 \\
\hline TABLE4 & 0 & 4992 & 2464 & $4.36423 \mathrm{D}+04$ & $1.3642 \mathrm{D}-11$ & $1.6488 \mathrm{D}-09$ & 28 & 27 & 7 & 0.61 & 2.42 \\
\hline TABLE5 & 0 & 4992 & 2464 & $4.36423 \mathrm{D}+04$ & $1.3642 \mathrm{D}-11$ & $1.6488 \mathrm{D}-09$ & 28 & 27 & 7 & 0.61 & 2.41 \\
\hline TABLE6 & 0 & 1584 & 510 & $3.70607 \mathrm{D}+05$ & $4.6566 \mathrm{D}-10$ & $9.6468 \mathrm{D}-09$ & 22 & 21 & 6 & 0.05 & 0.11 \\
\hline TABLE7 & 0 & 624 & 230 & $5.95773 \mathrm{D}+04$ & $6.0822 \mathrm{D}-12$ & $1.8668 \mathrm{D}-09$ & 23 & 22 & 7 & 0.02 & 0.05 \\
\hline TABLE8 & 0 & 1271 & 72 & $1.89571 \mathrm{D}+00$ & $1.0780 \mathrm{D}-14$ & $9.6495 \mathrm{D}-09$ & 16 & 15 & 6 & 0.01 & 0.06 \\
\hline TAME & 0 & 2 & 1 & $0.00000 \mathrm{D}+00$ & $0.0000 \mathrm{D}+00$ & $2.5059 \mathrm{D}-09$ & 1 & 4 & 6 & 0.00 & 0.00 \\
\hline TARGUS & 0 & 162 & 63 & $1.08379 \mathrm{D}+03$ & $4.5475 \mathrm{D}-13$ & $3.5821 \mathrm{D}-09$ & 18 & 17 & 6 & 0.01 & 0.01 \\
\hline TOYSARAH & 1 & 2890 & 1649 & $5.69809 \mathrm{D}+09$ & $8.7808 \mathrm{D}-05$ & $3.1275 \mathrm{D}-09$ & 126 & 115 & 6 & 2.26 & 1.75 \\
\hline TWO5IN6 & 0 & 5681 & 9629 & $4.59307 \mathrm{D}+03$ & $1.9714 \mathrm{D}-09$ & $2.6969 \mathrm{D}-09$ & 14 & 13 & 6 & 0.45 & 1.70 \\
\hline TWOD & -50 & 70521 & 67080 & - & - & - & - & - & - & 7200.00 & - \\
\hline UBH1 & 0 & 9009 & 6000 & $1.11600 \mathrm{D}+00$ & $1.2496 \mathrm{D}-12$ & $2.5061 \mathrm{D}-09$ & 8 & 7 & 6 & 24.34 & 0.21 \\
\hline WATER & 0 & 31 & 10 & $1.05493 \mathrm{D}+04$ & $1.7053 \mathrm{D}-13$ & $2.5078 \mathrm{D}-09$ & 14 & 13 & 6 & 0.00 & 0.00 \\
\hline ZANGWIL3 & 0 & 3 & 3 & $0.00000 \mathrm{D}+00$ & $5.3291 \mathrm{D}-14$ & $0.0000 \mathrm{D}+00$ & 1 & 0 & 0 & 0.00 & 0.00 \\
\hline
\end{tabular}

Para o conjunto testado, em

- 179 problemas, um ponto aproximadamente estacionário foi encontrado;

- 6 problemas, um ponto aproximadamente estacionário e quase viável foi encontrado;

- 6 problemas, o conjunto viável era vazio;

- 3 problemas, o máximo de iterações externas foi excedido;

- 2 problemas, houve falta de progresso por máximo de iterações internas;

- 1 problema, os iterandos divergiram;

- 3 problemas, o tempo máximo de duas horas de execução foi excedido.

Em geral, o desempenho do método neste conjunto de problemas testados foi satisfatório. Nos problemas em que o tempo de execução foi excedido, podemos notar que todo o tempo foi consumido na fase de pré-processamento, isto é, LCMIN não foi capaz de encontrar um ponto inicial viável e interior para começar a fase de otimização, provavelmente porque não exista um tal ponto.

\subsection{Parte II - Análise comparativa}

Numa segunda etapa, rodamos ALGENCAN [1, 2] versão 3.0.0 e IPOPT [25] versão 3.11.7 (as mais recentes até a presente data $[7,18]$ ) com os mesmos 200 problemas, e fizemos uma análise comparativa. IPOPT foi rodado com a opção bound_relax_factor 0 , isto é, não permitimos que IPOPT perturbe as restrições de caixa dadas pelo problema. Tomamos esta decisão por acreditar que não é bom relaxar as restrições do problema em nenhuma hipótese. Se por um lado isto facilita a resolução de um problema do ponto de vista numérico, por outro pode prejudicar drasticamente a solução caso o ponto final encontrado esteja muito próximo de alguma borda. Isto porque, neste caso, o ponto será inviável para o problema original e, portanto, não significa nada para o problema. Não obstante, ainda que o ponto final seja projetado na caixa, tal projeção pode fazer com que o 
ponto projetado perca a otimalidade. Mais ainda, esta é a forma de comparação mais justa com nossa implementação, em que optamos por não relaxar as restrições de caixa.

Para tal análise, seguimos algumas ideias de [6] e consideramos que um método resolveu um problema se o ponto final $\bar{x}$ é viável, isto é, satisfaz (4.1), e

$$
\frac{f(\bar{x})-f_{\min }}{\max \left\{1,\left|f_{\min }\right|\right\}} \leq 10^{-4}
$$

onde $f_{\text {min }}$ é o menor valor de função encontrado para um dado problema entre os valores que os métodos que estão sendo comparados convergiram. O valor da constante $10^{-4}$ é, de certa forma, arbitrário. Para diferentes valores, pode ser que os resultados se alterem. Uma discussão mais apurada sobre isso pode ser encontrada em [6]. Além disso, consideraremos que o método não é aplicável para um dado problema se ele não for capaz sequer de iniciar sua execução, parando por algum motivo. Assim sendo, para nossas comparações, denotaremos

$$
\begin{array}{ll}
\text { fun: } & \text { o valor da função objetivo não satisfaz (4.2). } \\
\text { inv: } & \text { convergência a um ponto inviável } \\
\text { nap: } & \text { não aplicável } \\
\text { sol: } & \text { solução ótima encontrada } \\
\text { tot: } & \text { total }
\end{array}
$$

Nesta fase, exibiremos os resultados da seguinte forma. Primeiro, colocaremos numa tabela um comparativo, problema a problema, do que aconteceu entre LCMIN e IPOPT e LCMIN e ALGENCAN. Com base nestes resultados, exibiremos perfis de desempenho [13] do tempo de execução dos métodos para resolução dos problemas. Numa primeira abordagem, atribuímos tempo infinito àqueles problemas que um método não resolveu. Desta forma, estaremos dando ênfase à robustez, isto é, é possível ver, no perfil de desempenho, qual a porcentagem de problemas que cada método resolveu no total. Contudo, as informações sobre eficiência podem não ser tão fieis. Por este motivo, numa segunda abordagem, exibiremos também os perfis de desempenho incluindo apenas os problemas que todos os métodos que estão comparados resolveram. Desta forma, estaremos exibindo um resultado fiel à eficiência de cada método. Por fim, exibiremos perfis de desempenho mostrando uma comparação entre os três métodos.

Vale dizer que para alguns problemas do conjunto teste, é comum que os métodos executem de maneira tão rápida que o cronômetro do computador não seja capaz de marcar o tempo exato de execução, retornando tempo zero. Mais especificamente, dos 200 problemas testados, isso aconteceu 31 vezes com ALGENCAN, 39 vezes com IPOPT e 34 vezes com LCMin. Nestes casos, substituímos o tempo zero por 0.01 .

Na Tabela 4.2, exibimos o resultado comparativo entre LCMIN e IPOPT.

\begin{tabular}{|c||c|c|c||c|}
\multicolumn{9}{|c||}{ LCMIN } \\
\hline \hline & sol & inv & fun & tot \\
\hline \hline sol & 148 & 5 & 8 & 161 \\
\hline inv & - & 7 & - & 7 \\
\hline fun & 9 & - & - & 9 \\
\hline nap & 19 & 4 & - & 23 \\
\hline tot & 176 & 16 & 8 & $\mathbf{2 0 0}$ \\
\hline
\end{tabular}

Tabela 4.2: Tabela comparativa entre LCMIN e IPOPT.

Nestes resultados, podemos notar que LCMIN e IPOPT resolveram 148 problemas em comum. No total, LCMIN resolveu 176 problemas, enquanto IPOPT resolveu 161. Em particular, IPOPT não 
foi aplicável em 23 problemas, onde $m>n$, dos quais em 19 LCMIN encontrou uma solução ótima e em 4 constatou que o conjunto viável era vazio. Nestes casos, nosso método elimina as restrições linearmente dependentes de $A$, satisfazendo a necessidade de $A$ possuir posto completo para resolver os sistemas lineares que a envolve. A não ser por esses casos, os métodos são bastante similares.

Na Tabela 4.3, exibimos o resultado comparativo entre LCMIN e ALGENCAN.

\begin{tabular}{|c|c|c|c|c|c|}
\hline & & & $\mathrm{CMI}$ & & \\
\hline & & sol & inv & fun & tot \\
\hline 8 & sol & 159 & 1 & 10 & 170 \\
\hline 1 & inv & 13 & 15 & - & 28 \\
\hline & fun & 2 & - & - & 2 \\
\hline & tot & 174 & 16 & 10 & 200 \\
\hline
\end{tabular}

Tabela 4.3: Tabela comparativa entre LCMIN e ALGENCAN.

Nestes resultados, podemos notar que LCMIN e ALGENCAN resolveram 159 problemas em comum. No total, LCMin resolveu 174 problemas, enquanto ALGEnCAN, 170. Temos também que, para 13 problemas que LCMIN resolveu, ALGENCAN convergiu a um ponto inviável. Isso, em geral, aconteceu quando ALGENCAN não convergiu a um ponto aproximadamente estacionário do problema, parando por algum critério de falta de progresso. De qualquer forma, os resultados foram bastante similares.

Na Figura 4.1, exibimos perfis de desempenho comparando o tempo de execução dos métodos. Neles, podemos notar como LCMIN se sobressai em robustez e em eficiência quando comparado ao ALGENCAN, e como LCMIN se sobressai em robustez, todavia perde ligeiramente em eficiência nas primeiras unidades de tempo quando comparado ao IPOPT.

Na Figura 4.2, exibimos os perfis de desempenho de tempo de execução considerando apenas os 159 problemas que ambos ALGENCAN e LCMIN resolveram, e os 148 problemas que ambos IPOPT e LCMIN resolveram. Nestes, podemos notar como LCMIN ainda é um pouco menos eficiente que IPOPT (até a décima unidade de tempo, aproximadamente). Ainda assim, o método mostra-se competitivo.

Por fim, na Figura 4.3, mostramos os perfis de desempenho comparando o tempo de execução de ALGENCAN, IPOPT e LCMIN. Mostramos os resultados para os 200 problemas do conjunto teste, atribuindo tempo infinito àqueles problemas que um método não foi capaz de resolver, e mostramos também o resultado comparativo para os 134 problemas que os três métodos resolveram. Neles, podemos notar como o LCMIN mostra-se competitivo. 


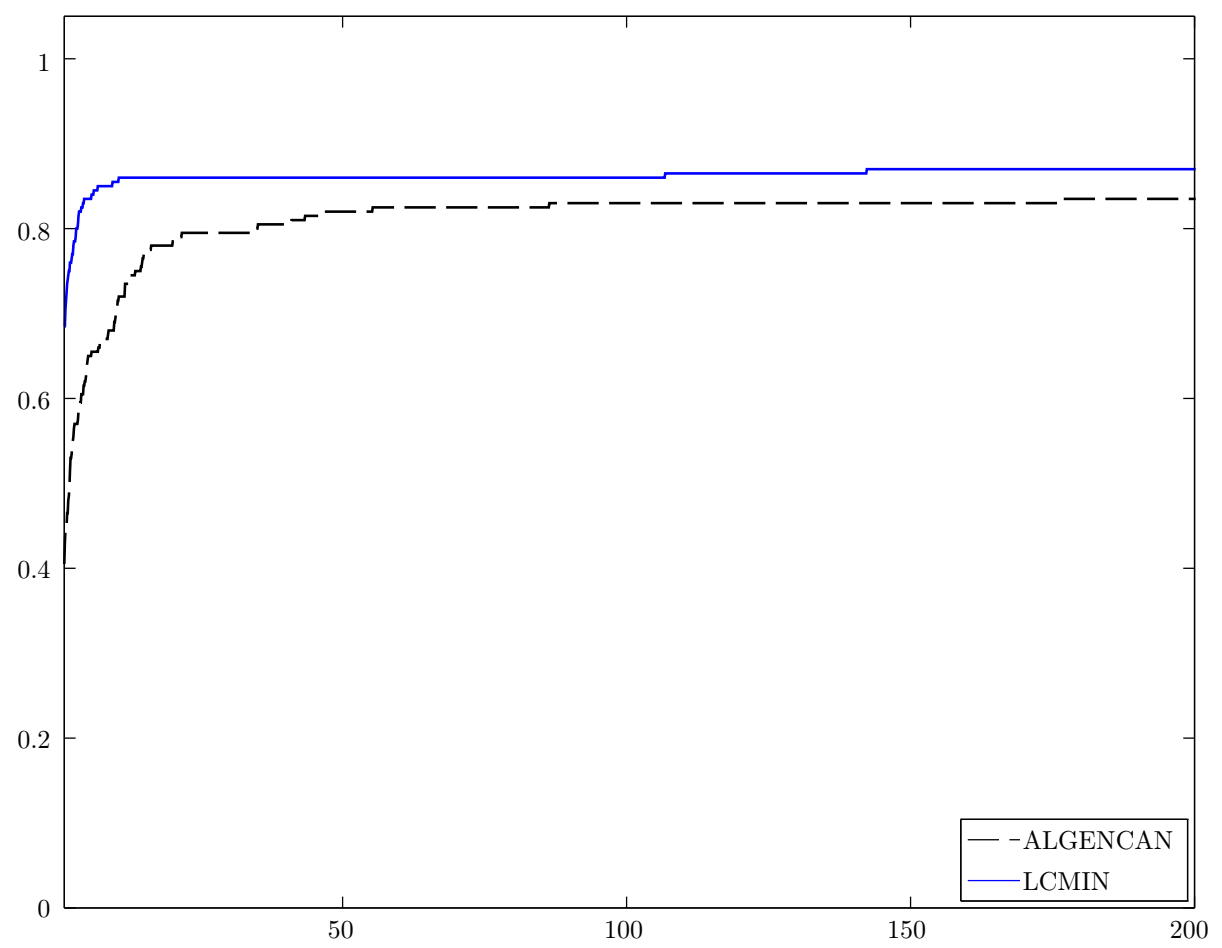

(a) Perfil de desempenho entre ALGenCAn e LCMin.

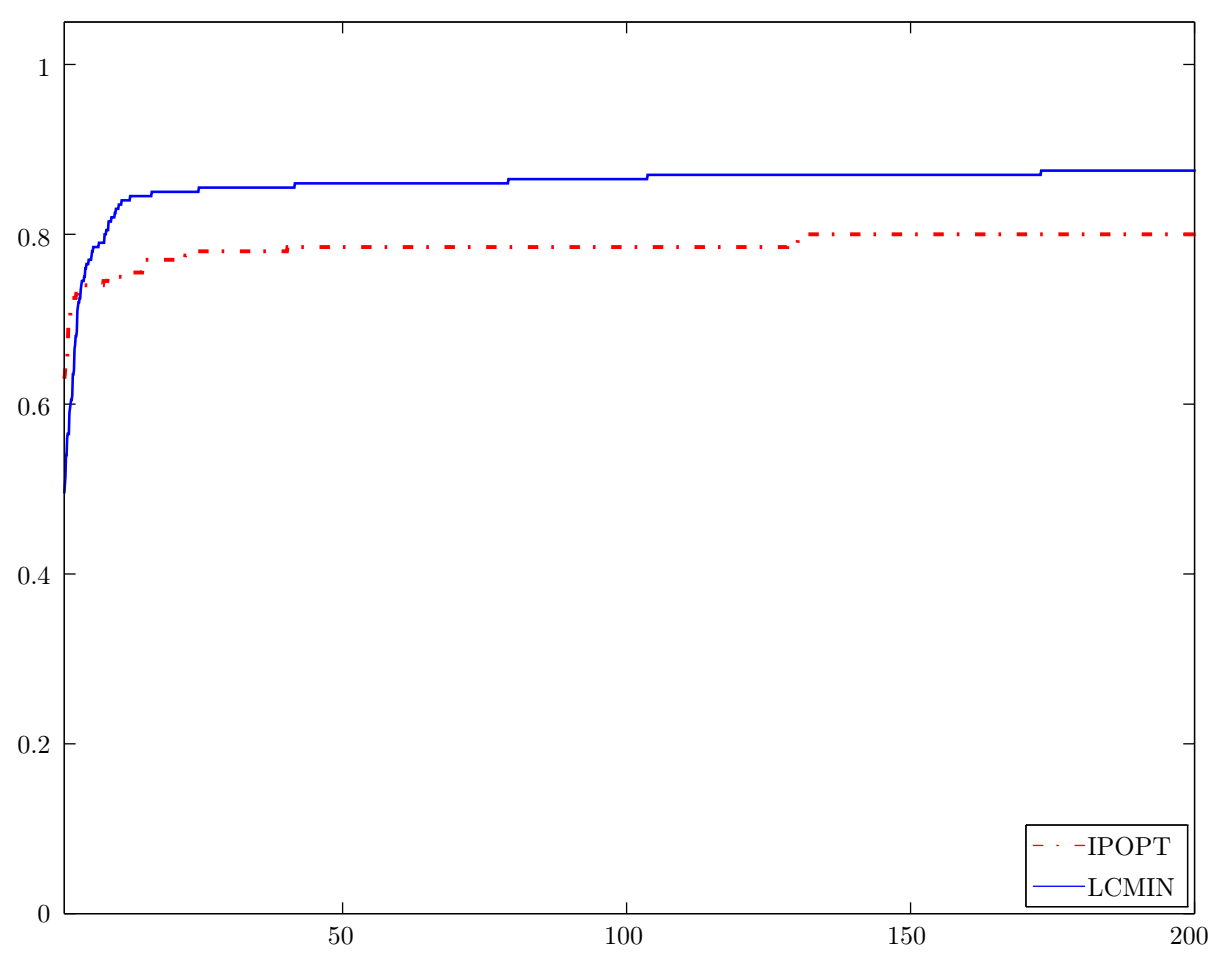

(b) Perfil de desempenho entre IPOPT e LCMIN.

Figura 4.1: Perfis de desempenho de tempo de execução de ALGENCAN e IPOPT comparados ao LCMIN. 


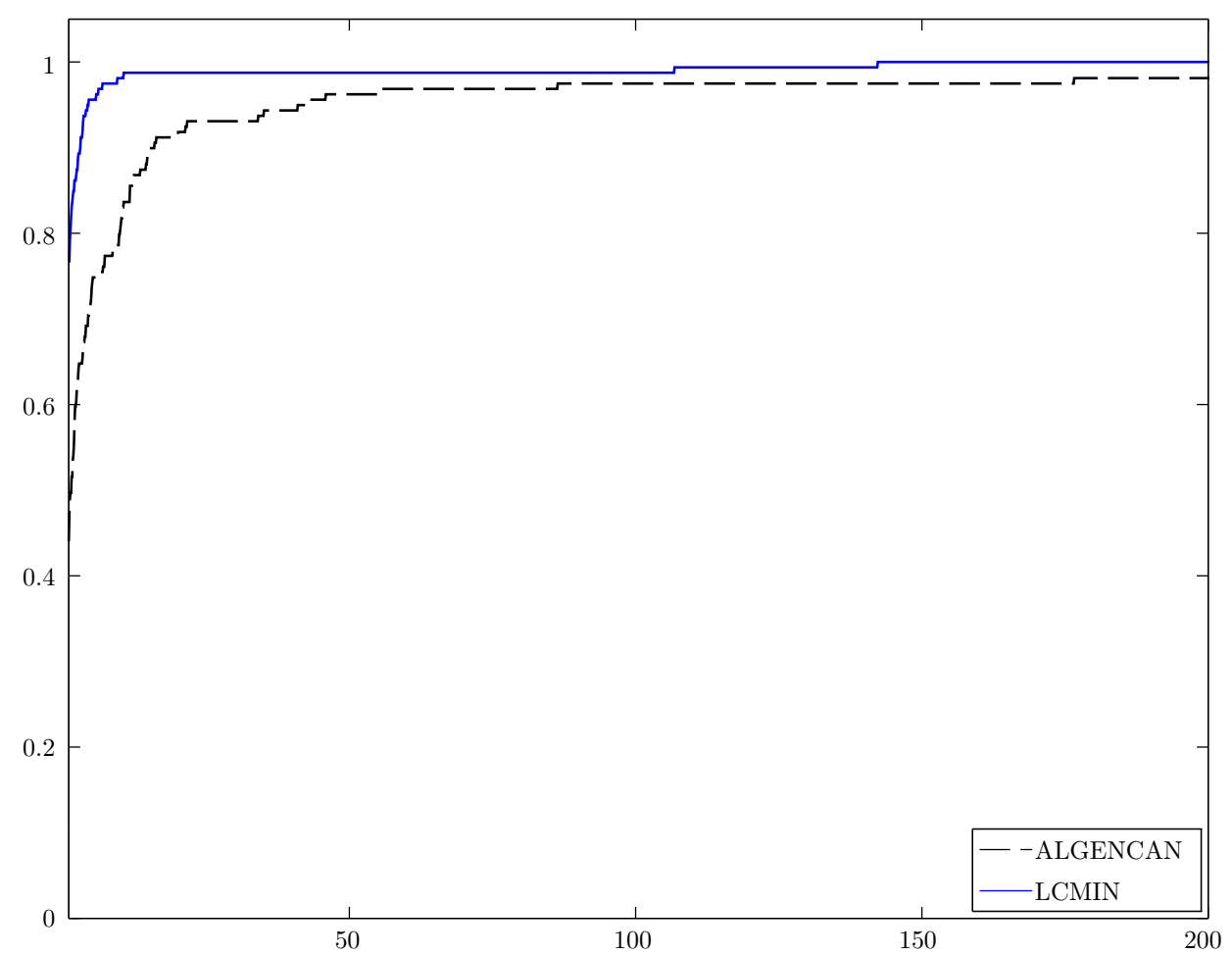

(a) Perfil considerando os 159 problemas que ambos ALGENCAN e LCMIN resolveram.

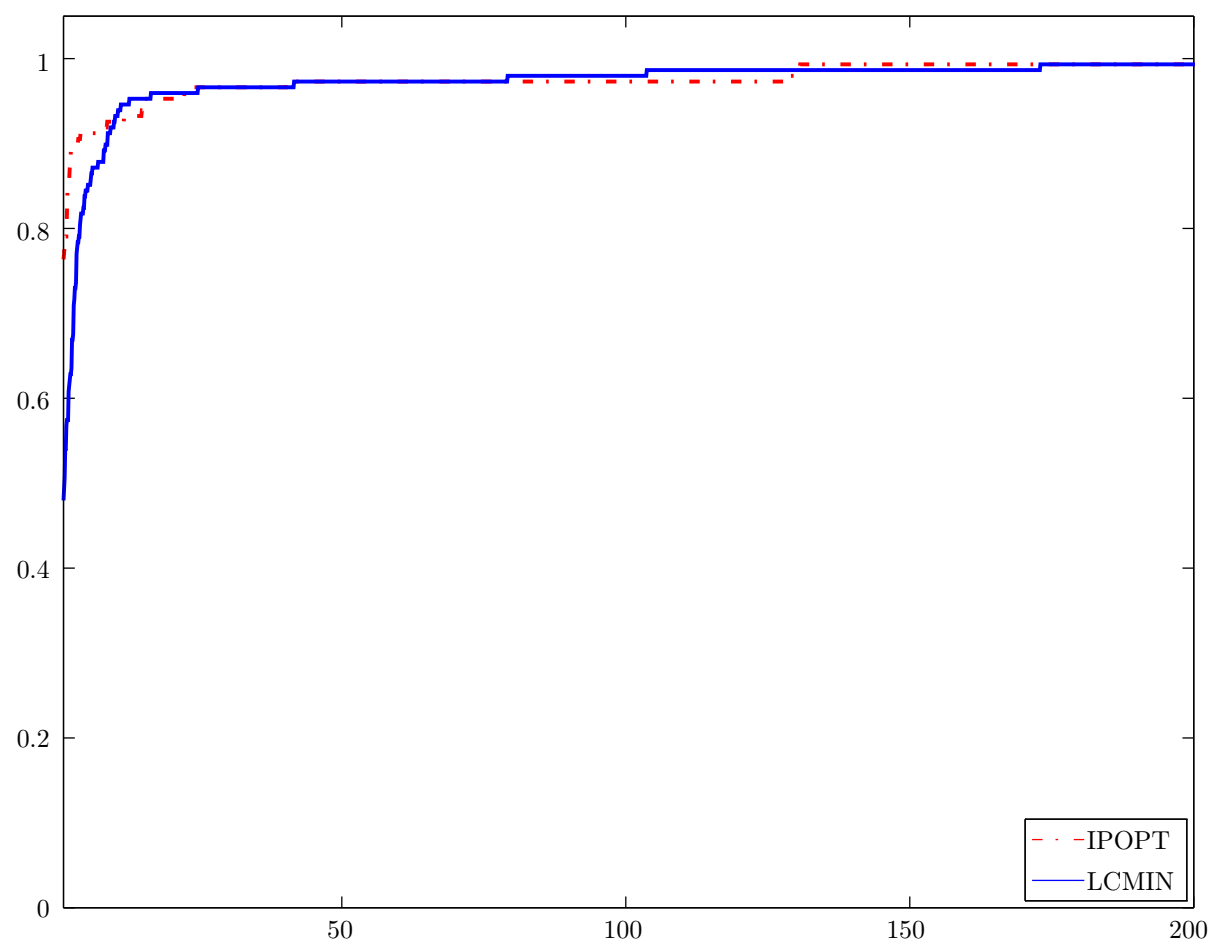

(b) Perfil considerando os 148 problemas que ambos IPOPT e LCMIN resolveram.

Figura 4.2: Perfis de desempenho de tempo de execução de ALGENCAN e IPOPT comparados ao LCMIN. Nestes perfis, consideramos apenas os problemas que ambos os métodos resolveram. 


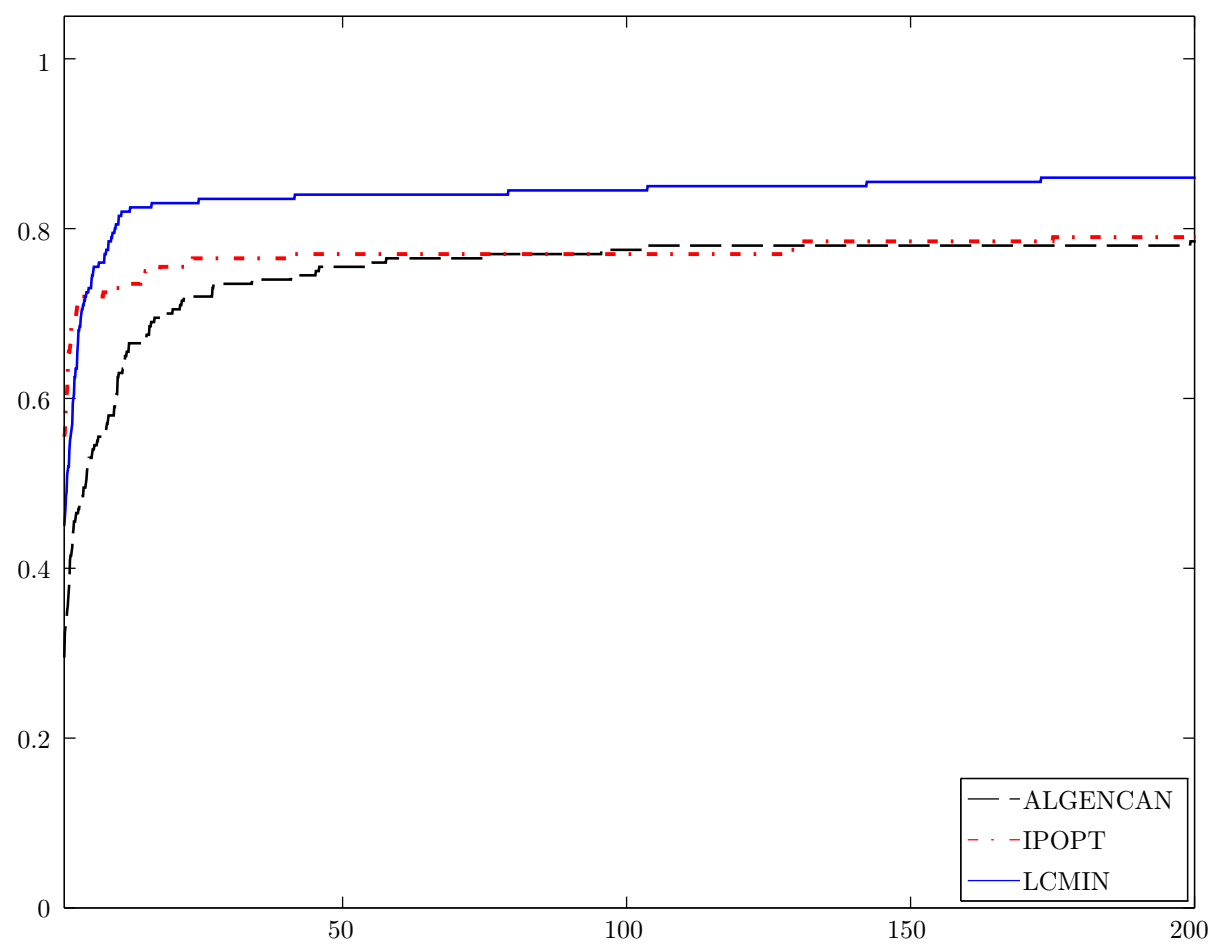

(a) Perfil de desempenho considerando os 200 problemas do conjunto teste.

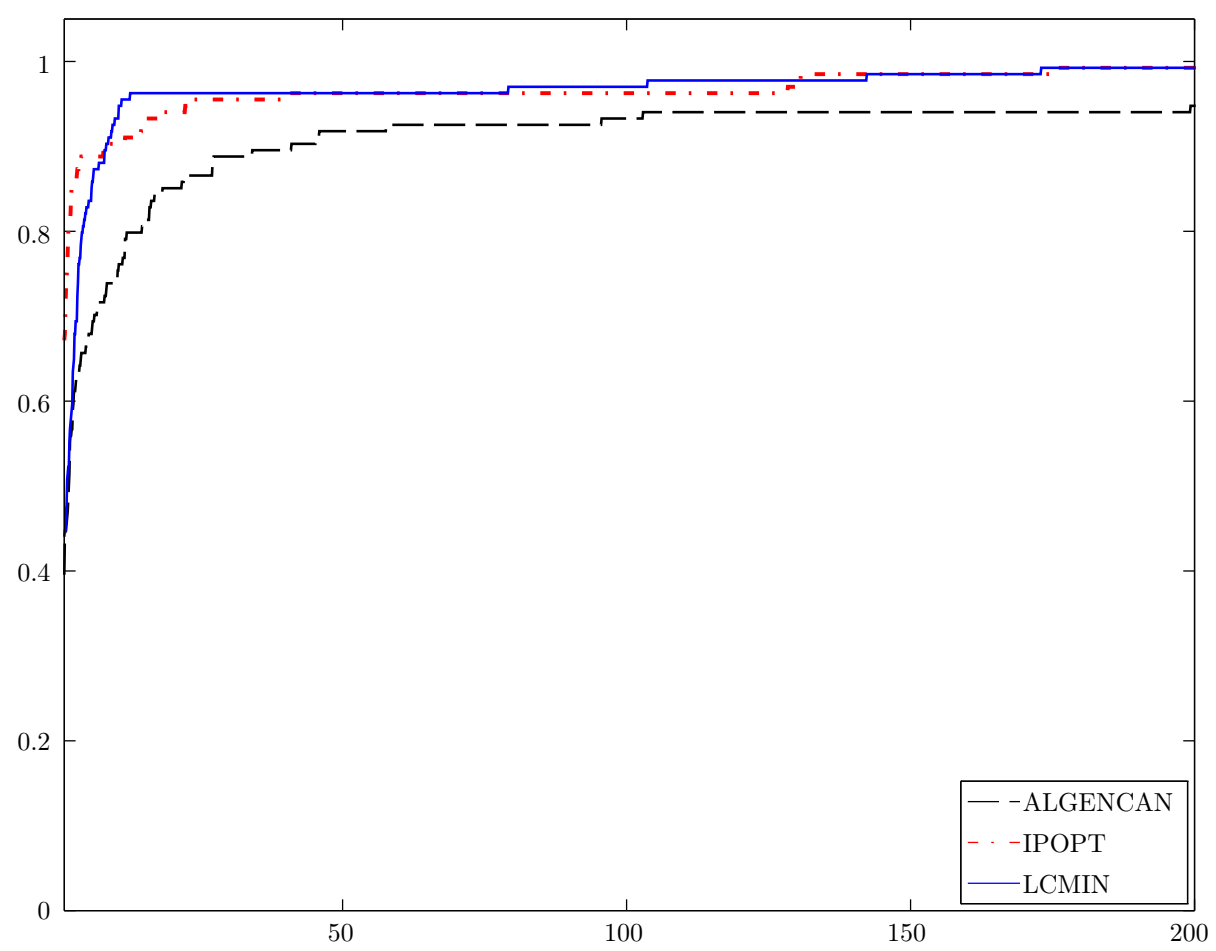

(b) Perfil de desempenho considerando apenas os 134 problemas que foram resolvidos por todos os métodos.

Figura 4.3: Perfis de desempenho de tempo de execução entre ALGENCAN, IPOPT e LCMIN. 


\section{Capítulo 5}

\section{Considerações finais e trabalhos futuros}

Neste trabalho, propusemos um método de pontos interiores primal-dual viável para minimização com restrições lineares. A principal diferença é que a otimização é iniciada com um ponto inicial que satisfaz as restrições lineares e de caixa estritamente, e ao longo do processo a viabilidade dos iterandos é mantida. Neste contexto, nossa contribuição foi propor um método que não pressupõe que o usuário forneça um ponto com tais características, mas consiste num método prático em que o ponto inicial é calculado como parte de uma fase de pré-processamento. Além disso, para o método proposto, mostramos os resultados de convergência global e experimentos numéricos com problemas clássicos da literatura, que atestaram a validade e a competitividade da implementação.

Por um lado, nosso objetivo era verificar se faz sentido, no contexto de pontos interiores, um método que preserva a viabilidade dos iterandos; chegamos à conclusão de que um tal método pode ser competitivo. Por outro lado, a questão que emerge naturalmente no contexto de otimização de grande porte é a possibilidade de resolução dos sistemas lineares envolvidos usando métodos indiretos, particularmente útil quando a fatoração de matrizes torna-se impraticável devido às dimensões do problema. A esta questão ainda não temos uma resposta fechada, ficando portanto como trabalho futuro. Além disso, queremos averiguar qual seria a vantagem do uso de outras estratégias de globalização, como regiões de confiança, no lugar de busca linear. Os próximos passos deste trabalho serão:

1. Avaliar a possibilidade de resolução dos sistemas lineares por métodos iterativos, isto é, que dispensem fatoração de matrizes,

2. Avaliar o uso de regiões de confiança no contexto de pontos interiores e otimização de grande porte $[22,23]$. 
Apêndices 



\section{Apêndice A}

\section{Condições de qualificação do problema}

Nesta seção, discutiremos uma propriedade adicional do problema (2.1), decorrente da Hipótese 1, essencial para a abordagem de pontos interiores, nomeadamente que a condição de qualificação de Mangasarian-Fromovitz (MFCQ) vale para todo ponto viável do problema. Começaremos com a seguinte definição.

Definição 18. Consideremos o problema de programação não linear

$$
\begin{aligned}
\min & f(x) \\
\text { s.a } & h(x)=0 \\
& g(x) \leq 0,
\end{aligned}
$$

onde $f: \mathbb{R}^{n} \rightarrow \mathbb{R}, h: \mathbb{R}^{n} \rightarrow \mathbb{R}^{m}$ e $g: \mathbb{R}^{n} \rightarrow \mathbb{R}^{p}$ funções contínuas e diferenciáveis e consideremos $x$ um ponto viável deste problema. Consideremos ainda

$$
\mathcal{A}(x)=\left\{i \mid g_{i}(x)=0\right\} .
$$

Dizemos que a condição de qualificação de Mangasarian-Fromovitz (MFCQ) vale em $x$ se

$$
\left\{\nabla h_{1}(x), \nabla h_{2}(x), \ldots, \nabla h_{m}(x)\right\}
$$

for um conjunto de vetores linearmente independentes e existir um vetor $d \in \mathbb{R}^{n}$ tal que $\nabla h_{i}(x)^{T} d=$ 0 , para todo $i=1, \ldots, m$, e $\nabla g_{k, j}(x)^{T} d<0$, para todo $j \in \mathcal{A}(x)$.

A Definição 18 é feita acerca de um problema geral de programação não linear. Do ponto de vista geométrico, MFCQ diz que, para um dado ponto viável $x$, existe uma direção não nula no núcleo do Jacobiano da $h(x)$, isto é, que preserva a viabilidade de primeira ordem de $\{x \mid h(x)=0\}$, e que aponta para o conjunto $\{x \mid g(x)<0\}$. Em particular, para nosso problema (2.1), temos que MFCQ vale num ponto $x$ sempre que $x$ for viável, $A$ possuir posto linha completo e existir uma direção $d \in \mathbb{R}^{n}$ tal que $d$ esteja no núcleo da matriz $A$ e $d_{i}>0$ se $x_{i}=\ell_{i}$ e $d_{i}<0$ se $x_{i}=u_{i}$.

Mostramos, no resultado que segue, que a Hipótese 1 garante que MFCQ vale em todos os pontos viáveis do problema $(2.1)$.

Proposição 19. Sob a Hipótese 1, todo ponto viável do problema (2.1) satisfaz MFCQ.

Demonstração. Se $x \in \mathcal{F}_{0}$, então MFCQ vale, pois, neste caso, $\mathcal{A}(x)=\emptyset \mathrm{e}$, como $x$ é viável, basta tomar qualquer $d$ no núcleo da $A$. Esta $d$ existe, já que $A x=b$ é compatível e $m<n$. Consideremos então um ponto viável $x$ qualquer tal que $x \notin \mathcal{F}_{0}$. Seja $d=\bar{x}-x$, com $\bar{x} \in \mathcal{F}_{0}$ (a existência de $\bar{x}$ é garantida pela Hipótese 1). Primeiramente, $A d=A(\bar{x}-x)=b-b=0$, ou seja, $d$ está no núcleo 
da $A$. Mais ainda, se $x_{i}=\ell_{i}$, temos que $d_{i}=\bar{x}_{i}-x_{i}=\bar{x}_{i}-\ell_{i}>0$ e, analogamente, se $x_{i}=u_{i}$, temos que $d_{i}=\bar{x}_{i}-x_{i}=\bar{x}_{i}-u_{i}<0$. Portanto, a condição MFCQ é satisfeita em qualquer ponto viável do problema (2.1).

O objetivo agora é mostrar, para o nosso problema, que para todo $x$ que satisfaz as condições KKT (2.3) do problema (2.1), o conjunto de multiplicadores de Lagrange associados é limitado. Isto é consequência direta do resultado da Proposição 19, que implica que todo ponto viável satisfaz MFCQ. Começaremos pela seguinte proposição, onde é estabelecida uma condição suficiente de falha de MFCQ, que nada mais é que uma particularização da forma dual de MFCQ [24].

Proposição 20. Seja $x \in \mathbb{R}^{n}$ um ponto viável do problema (2.1). Se existirem vetores $\lambda \in \mathbb{R}^{m}$, $\nu_{\ell} \in \mathbb{R}_{+}^{n}$ e $\nu_{u} \in \mathbb{R}_{+}^{n}$ não todos nulos tais que

$$
\begin{aligned}
& A^{T} \lambda-\nu_{\ell}+\nu_{u}=0, \\
& {\left[\nu_{\ell}\right]_{i}=0 \text { se } x_{i}>\ell_{i}}
\end{aligned}
$$

$e$

$$
\left[\nu_{u}\right]_{i}=0 \text { se } x_{i}<u_{i}
$$

então MFCQ não vale em $x$.

Demonstração. A fim de derivar uma contradição, suponhamos que existe um ponto viável $x \in \mathbb{R}^{n}$ do problema (2.1) tal que MFCQ vale e existem vetores $\lambda \in \mathbb{R}^{m}, \nu_{\ell} \in \mathbb{R}_{+}^{n}$ e $\nu_{u} \in \mathbb{R}_{+}^{n}$ não todos nulos que satisfazem (A.1-A.3).

Como $x$ satisfaz MFCQ, existe $d \in \mathbb{R}^{n}$ tal que $A d=0$ e

$$
d_{i}>0 \text { se } x_{i}=\ell_{i} \text { e } d_{i}<0 \text { se } x_{i}=u_{i} .
$$

Pré-multiplicando (A.1) por $d^{T}$ temos que

$$
d^{T} \nu_{u}-d^{T} \nu_{\ell}=0
$$

Por outro lado, notemos que $\left(\nu_{\ell}, \nu_{u}\right) \neq 0$. Caso contrário, (A.1) e a independência linear das linhas da $A$ implicariam $\lambda=0$, contradizendo que $\lambda, \nu_{\ell}$ e $\nu_{u}$ não são todos nulos. Logo, temos que $\left(\nu_{\ell}, \nu_{u}\right) \neq 0, \nu_{\ell} \geq 0, \nu_{u} \geq 0$ e (A.4) implicam que

$$
d^{T} \nu_{u}-d^{T} \nu_{\ell}<0
$$

o que contradiz (A.5).

O resultado que segue é baseado em [5, Exercício 3.3.19].

Proposição 21. Se vale $M F C Q$ em qualquer ponto $x \in \mathbb{R}^{n}$ que satisfaz as condições $K K T$ (2.3) do problema (2.1), então seu conjunto de multiplicadores de Lagrange é limitado.

Demonstração. Suponhamos, por absurdo, que valha MFCQ para qualquer ponto viável do problema (2.1) e que, no conjunto de multiplicadores associados a um ponto $x \in \mathbb{R}^{n}$ que satisfaz $\operatorname{KKT}(2.3)$, exista uma sequência $\left\{\lambda^{j}, \nu_{\ell}^{j}, \nu_{u}^{j}\right\}$, com $j \in \mathbb{N}$, tal que

$$
\lim _{j \rightarrow \infty}\left\|\lambda^{j}\right\|+\left\|\nu_{\ell}^{j}\right\|+\left\|\nu_{u}^{j}\right\|=\infty .
$$


Sem perda de generalidade, consideremos que $\left(\lambda^{j}, \nu_{\ell}^{j}, \nu_{u}^{j}\right) \neq(0,0,0)$ para todo $j$. De fato, se algum dos vetores for nulo para todo $j$, então podemos desconsiderá-lo, já que não contribui no limite (A.6). Em caso contrário, basta considerar $j$ suficientemente grande. Seja

$$
\beta^{j}=\frac{\lambda^{j}}{\left\|\lambda^{j}\right\|}, \gamma_{\ell}^{j}=\frac{\nu_{\ell}^{j}}{\left\|\nu_{\ell}^{j}\right\|} \text { e } \gamma_{u}^{j}=\frac{\nu_{u}^{j}}{\left\|\nu_{u}^{j}\right\|} .
$$

Como $\left(\lambda^{j}, \nu_{\ell}^{j}, \nu_{u}^{j}\right)$ são multiplicadores de Lagrange, então

$$
\left[\gamma_{\ell}^{j}\right]_{i}=0 \text { se } x_{i}>\ell_{i},\left[\gamma_{u}^{j}\right]_{i}=0 \text { se } x_{i}<u_{i} \text { e }\left[\gamma_{\ell}^{j}\right]_{i},\left[\gamma_{u}^{j}\right]_{i} \geq 0 \text {, para todo } i=1, \ldots, n \text {. }
$$

Consideremos a combinação $A^{T} \beta^{j}-\gamma_{\ell}^{j}+\gamma_{u}^{j}$. Temos que

$$
\begin{aligned}
A^{T} \beta^{j}-\gamma_{\ell}^{j}+\gamma_{u}^{j} & =A^{T} \frac{\lambda^{j}}{\left\|\lambda^{j}\right\|}-\frac{\nu_{\ell}^{j}}{\left\|\nu_{\ell}^{j}\right\|}+\frac{\nu_{u}^{j}}{\left\|\nu_{u}^{j}\right\|} \\
& =\frac{A^{T}\left(1+\frac{\left\|\nu_{\ell}^{j}\right\|}{\left\|\lambda^{j}\right\|}+\frac{\left\|\nu_{u}^{j}\right\|}{\left\|\lambda^{j}\right\|}\right)-\nu_{\ell}^{j}\left(\frac{\left\|\lambda^{j}\right\|}{\left\|\nu_{\ell}^{j}\right\|}+1+\frac{\left\|\nu_{u}^{j}\right\|}{\left\|\nu_{\ell}^{j}\right\|}\right)+\nu_{u}^{j}\left(\frac{\left\|\lambda^{j}\right\|}{\left\|\nu_{u}^{j}\right\|}+\frac{\left\|\nu_{\ell}^{j}\right\|}{\left\|\nu_{u}^{j}\right\|}+1\right)}{\left\|\lambda^{j}\right\|+\left\|\nu_{\ell}^{j}\right\|+\left\|\nu_{u}^{j}\right\|},
\end{aligned}
$$

donde segue, junto com (A.6), que

$$
\lim _{j \rightarrow \infty} A^{T} \beta^{j}-\gamma_{\ell}^{j}+\gamma_{u}^{j}=0
$$

Portanto, para qualquer $\left(\beta, \gamma_{\ell}, \gamma_{u}\right)$ ponto limite da sequência $\left\{\beta^{j}, \gamma_{\ell}^{j}, \gamma_{u}^{j}\right\}$, temos que

$$
A^{T} \beta-\gamma_{\ell}+\gamma_{u}=0 .
$$

Como $\left(\beta, \gamma_{\ell}, \gamma_{u}\right) \neq(0,0,0)$, então, pela Proposição 20, (A.7) e (A.8) implicam que MFCQ não vale em $x$, um absurdo, já que $x$ é viável e, pela Proposição 19, MFCQ vale para qualquer ponto viável do problema (2.1).

Deste modo, as Proposições 19 e 21 implicam o seguinte resultado.

Proposição 22. O conjunto de multiplicadores de Lagrange associado a qualquer ponto $x$ que satisfaz as condiçôes KKT (2.3) do problema (2.1) é limitado. 


\section{Referências Bibliográficas}

[1] R. Andreani, E. G. Birgin, J. M. Martínez, e M. L. Schuverdt. Augmented Lagrangian methods under the constant positive linear dependence constraint qualification. Mathematical Programming, 111(1-2):5-32, 2008.

[2] R. Andreani, E. G. Birgin, J. M. Martínez, e M. L. Schuverdt. On Augmented Lagrangian methods with general lower-level constraints. SIAM Journal on Optimization, 18(4):1286-1309, 2008 .

[3] M. Andretta, E. G. Birgin, e J. M. Martínez. Practical active-set Euclidian trust-region method with spectral projected gradients for bound-constrained minimization. Optimization, 54(3):305$325,2005$.

[4] H. Y. Benson, R. J. Vanderbei, e D. F. Shanno. Interior-point methods for nonconvex nonlinear programming: filter methods and merit functions. Computational Optimization and Applications, 23(2):257-272, 2002.

[5] D. P. Bertsekas. Nonlinear Programming. Athena Scientific, Belmont, US, 2 ed., 2008.

[6] E. G. Birgin e J. M. Gentil. Evaluating bound-constrained minimization software. Computational Optimization and Applications, 53(2):347-373, 2012.

[7] E. G. Birgin e J. M. Martínez. TANGO: Trustable Algorithms for Nonlinear General Optimization. http://www. ime.usp.br/ egbirgin/tango.

[8] E. G. Birgin e J. M. Martínez. A box-constrained optimization algorithm with negative curvature directions and spectral projected gradients. Em G. Alefeld e X. Chen, eds., Topics in Numerical Analysis, volume 15 de Computing Supplementa, pp. 49-60. Springer Vienna, 2001.

[9] E. G. Birgin e J. M. Martínez. Large-scale active-set box-constrained optimization method with spectral projected gradients. Computational Optimization and Applications, 23(1):101$125,2002$.

[10] R. H. Byrd, J. C. Gilbert, e J. Nocedal. A trust region method based on interior point techniques for nonlinear programming. Mathematical Programming, 89(1):149-185, 2000.

[11] R. H. Byrd, J. Nocedal, e R. A. Waltz. Knitro: an integrated package for nonlinear optimization. Em G. Pillo e M. Roma, eds., Large-Scale Nonlinear Optimization, volume 83 de Nonconvex Optimization and Its Applications, pp. 35-59. Springer US, 2006.

[12] L. Chen e D. Goldfarb. Interior-point $\ell_{2}$-penalty methods for nonlinear programming with strong global convergence properties. Mathematical Programming, 108(1):1-36, 2006.

[13] E. D. Dolan e J. J. Moré. Benchmarking optimization software with performance profiles. Mathematical Programming, 91(2):201-213, 2002. 
[14] T. M. R. Ellis, I. R. Philips, e T. M. Lahey. Fortran 90 Programming. Addison-Wesley, England, 1994.

[15] A. Forsgren, P. Gill, e M. Wright. Interior methods for nonlinear optimization. SIAM Review, 44(4):525-597, 2002.

[16] N. I. M. Gould. On practical conditions for the existence and uniqueness of solutions to the general equality quadratic programming problem. Mathematical Programming, 32(1):90-99, 1985.

[17] N. I. M. Gould, D. Orban, e Ph. L. Toint. CUTEst: a Constrained and Unconstrained Testing Environment with safe threads. Relatório técnico, Rutherford Appleton Laboratory Chilton, Oxfordshire, England, EU, 2013.

[18] COIN-OR Initiative. Ipopt. https://projects.coin-or.org/Ipopt.

[19] Harwell Subroutine Library. A collection of fortran codes for large scale scientific computation. http://www.hsl.rl.ac.uk, 2013.

[20] M. Metcalf, J. Reid, e M. Cohen. Modern Fortran explained. Oxford University Press, New York, 2011.

[21] J. Nocedal e S. J. Wright. Numerical Optimization. Springer, New York, 2 ed., 2006.

[22] M. Rojas, S. A. Santos, e D. C. Sorensen. A new matrix-free algorithm for the large-scale trust-region subproblem. SIAM Journal on Optimization, 11(3):611-646, 2001.

[23] M. Rojas, S. A. Santos, e D. C. Sorensen. Algorithm 873: LSTRS: MATLAB software for large-scale trust-region subproblems and regularization. ACM Trans. Math. Softw., 34(2):1$28,2008$.

[24] M. V. Solodov. Constraint qualifications. Em J. J. Cochran, L. A. Cox, P. Keskinocak, J. P. Kharoufeh, e J. C. Smith, eds., Wiley Encyclopedia of Operations Research and Management Science. John Wiley \& Sons, Inc., 2010.

[25] A. Wächter e L. T. Biegler. On the implementation of an interior-point filter line-search algorithm for large-scale nonlinear programming. Mathematical Programming, 106(1):25-57, 2006. 